\title{
A micro-scope on intracellular trafficking
}

\author{
PhD Thesis
}

in partial fulfillment of the requirements for the degree

“Doctor of Natural Sciences (Dr. rer. nat.)"

in the GGNB Program - Center of Molecular Physiology of the Brain

at the Georg August University Göttingen, Faculty of Biology

submitted by

\section{Christian Olendrowitz}

born in

Königs Wusterhausen, Germany 
I hereby declare that I prepared the PhD thesis:

\section{"A micro-scope on intracellular trafficking"}

on my own and with no other sources and aids than quoted.

Christian Olendrowitz

Göttingen, 10. May 2011

Thesis committee members:

Dr. Stefan Eimer, ENI Göttingen

Prof. Dr. Holger Stark, MPI-BPC Göttingen

Dr. Silvio Rizzoli, ENI Göttingen

Date of oral exam:

05.05 .2011

ii 
for my family 


\section{Acknowledgments}

First of all I would like to thank my supervisors Stefan Eimer and Tim Salditt for sharing their time, knowledge and enthusiasm for science in the last three years. Furthermore I would like to thank them for their patience and encouragement in times of need.

Secondly I would like to thank my thesis committee members Silvio Rizzoli and Holger Stark for their interest and guidance over the last years, especially in the early phases of the thesis work. All of these mentors criticism was essential in showing me how established scientists outside of the field of cellular trafficking perceive my work and how I should thus present it in an intelligible fashion.

I would also like to express my appreciation for all the members of the labs that I worked in during the last years. Special thanks in this regards are directed to the role model scientist Marija Sumakovic and Mandy Hannemann as well as the Lord of the Rabs Nikhil Sasidharan. I have to thank all of you for your friendship but also for your generous and self sacrificing support in the lab during the last years.

As invaluable guides in the world of x-ray physics I have to furthermore thank André Beerlink, Matthias Bartels, Robin Wilke and Sven-Philip Krüger. Without anyone of you and your useful comments this thesis in its current form would not have become possible.

Further thanks are addressed to our collaborating groups. I thank Bernard Lakowski for his support in the $d p y-1$ project but I would also like to thank Jon Audhya and Amber Schuh as well as Nektarios Tavernarakis and Nikos Kurtis whose project were not mentioned in this thesis. All of your projects have fascinated me and it was a pleasure to have worked with you.

Final thanks are addressed to the helping hands in the labs, scientist as well as technicians. To all of those, around or far away, who have supported me throughout the last years with their friendship. I share venturesome memories with all of you for which I am grateful beyond any measure. In this respect especially to mention are: David and Biese; Lukas, Hannes and Eike; Nora, Katrin and Ling; Erin; Christoph and Larus; as well as old friends like Andreas, Todd, Shim, Darwin, Gary, Thomas, as well as, Rolf and Fia together with their families. 
Last but not least I would like to thank my host family, my parents and grandparents for their unbroken support in the last years, as well as, Ulrike and her family. In this respect I would like to thank Ulrike especially for her unquestionable love and enduring patience. Thank you for keeping me sane and reminding me of the important things in life. 


\section{Abstract}

The general aim of this thesis work is to gain an encompassing insight into the intracellular trafficking machinery of multicellular organisms. For this purpose, the previously well-described model organism Caenorbabdits elegans is analyzed in our studies, with a focus on the family of small Rab-GTPases. These Rab-proteins (RABs) are the central players that orchestrate all intracellular trafficking events. An introduction to the current understandings in this field of science is given in the introductory chapter I. This thesis work began with a morphological description and a behavioral characterization of mutants of all members of rab-gene ( $r a b)$ family. The major technique used for this anatomic investigation on the cellular- and subcellular level was two- as well as three-dimensional electron microscopy of highpressure frozen nematode with a near-native sample-preservation. An atlas comprising all morphological data together with the results of additional behavioral studies, is the ultimate outcome of this thesis work. Among the descriptions of several tissues and organelles, the findings that the Golgi shapes seems to adapt to the activity in a tissue-specific manner is of central importance as it suggests that intracellular transport processes must also be investigated in a tissue-specific fashion. The generated tissue- and Rab-Atlas, along with all related results, is presented in chapter III, while a detailed description of used materials and methods is given in chapter II.

During the entire investigation a special focus was directed towards the ultra-fast trafficking events that ensure the transmission at synapses. To aid these studies, the image analysis tool XtraCOunt was generated during this thesis work in order to semi-automates the analysis of these trafficking studies. Chapter IV describes this tool along with all its features. In the atlas studies the rab-10 mutant was identified as potentially neuronally impaired. In chapter IV, this mutant is examined for its synaptic features and it has been found to resemble the rab-2 mutant alterations in dense-core vesicle biogenesis. XtraCOunt was conceived for the analysis of electron micrographs of nematode synapses but was shown to be applicable in a vast range of other trafficking studies. Current collaborating studies, using this tool, led to the understanding of the phenotype of Sydney Brenner's first described mutant $d p y-1$ (dumpy), whose cuticular components are not able to maintain their network integrity. The fundamental findings of this study are presented in chapter $\mathrm{V}$.

To overcome imaging-constrains presented by the mostly electron microscopic nature of my study, I have furthermore tested the possibility of studying high-pressure frozen specimen with x-rays. High resolution phase contrast imaging, holo-tomography as well as micro-fluorescence investigations were tested for their applicability, feasibility and limitations. Their underlying mathematical theory and the obtained experimental results are presented and discussed in chapter VI. The major results of these 
investigations is a full-worm-tomogram with subcellular resolution and a map of tissue-specific elemental distributions within worm thin-slices.

The concluding chapter VII briefly summarizes the results of all performed investigations in the context with one another and their possible meaning for the intracellular trafficking community. 


\section{Table of contents}

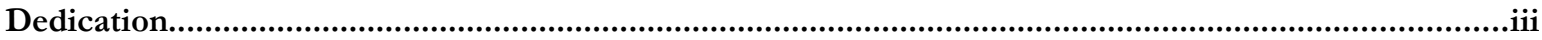

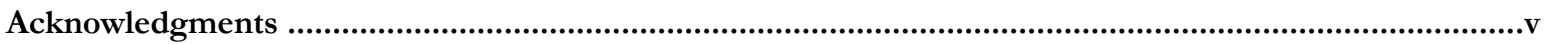

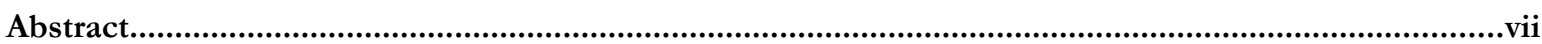





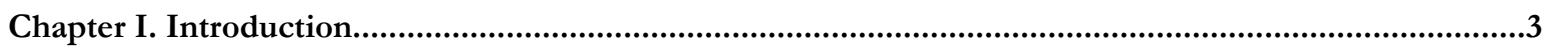

1.1 Eukaryotes tightly regulate their intracellular transport....................................................................................

1.2 Fission of membranes is supported and driven by multimolecular coats.......................................................

1.3 Target-specific fusion of membranes is mediated via SNARE proteins ..........................................................

1.4 Rab-GTPases localize compartment-specific to establish trafficking routes .....................................................

1.5 Rab-GTPases orchestrate trafficking through active recruitment of effectors...............................................10

1.6 Characterizing the entire Rab-family........................................................................................................... 14

1.7 The craft of preserving biological specimen for electron microscopy...........................................................16

1.8 Project description.



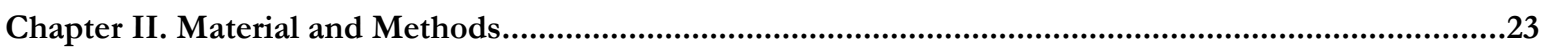

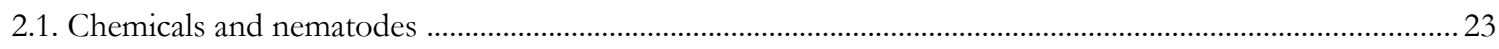

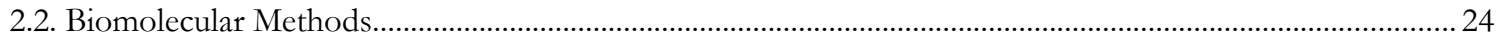

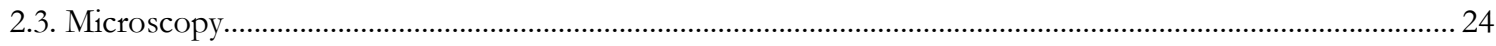

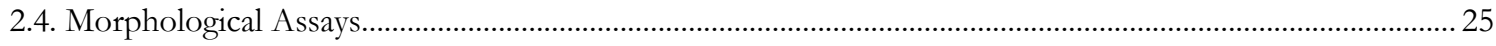

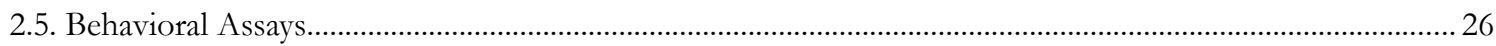

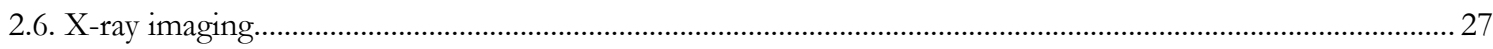

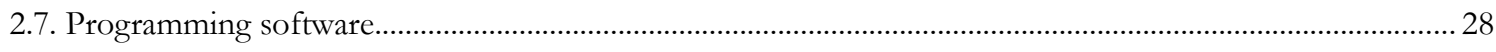






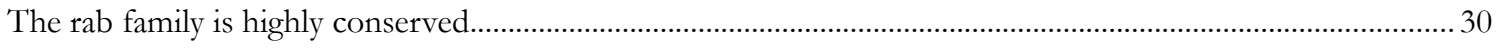

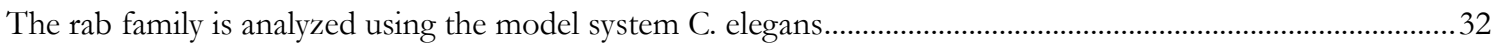

The preliminary analysis of rab mutants suggest individual and common roles of Rab-GTPases.....................33

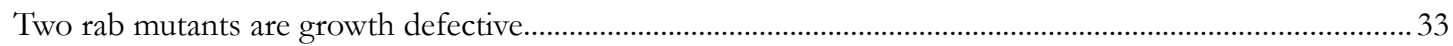

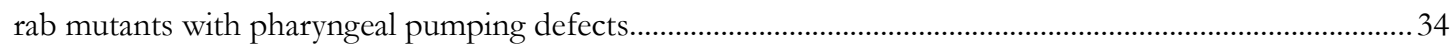

The defecation motor programs are defective in a number of rab mutants..................................................36

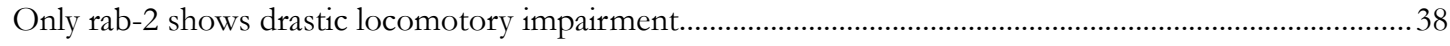

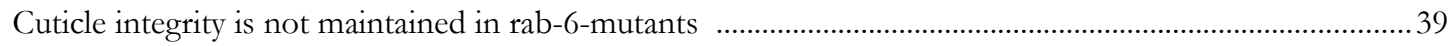

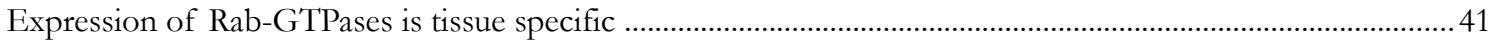

High Pressure Freezing preserves C. elegans tissues to an in vivo like state.....................................................42



Tissue reconstructions can be achieved with the help of serial sectioning..................................................44

The Golgi machinery has tissue specific adaptations.......................................................................................... 46

The subcellular anatomy can be analyzed with electron microscopy..............................................................46

The subcellular anatomy can be analyzed with electron microscopy...........................................................4

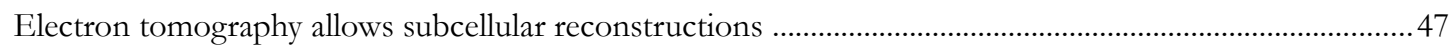

A open source Rab-Wiki comprises data from the Rab-Atlas study.................................................................49

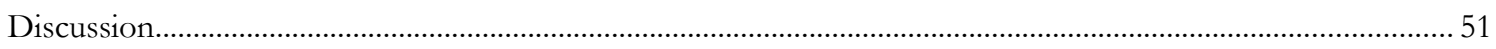

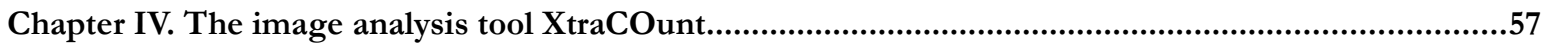

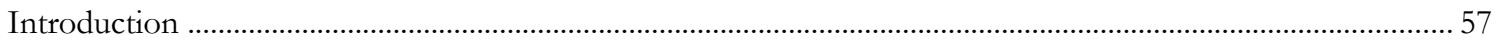

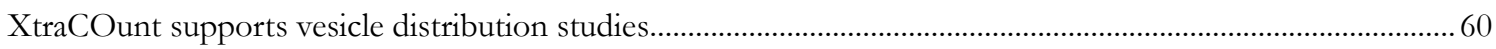

The program features of XtraCOunt are designed to be intuitive ....................................................................63

Analysis of distribution patterns in different model organisms with XtraCOunt ...........................................64

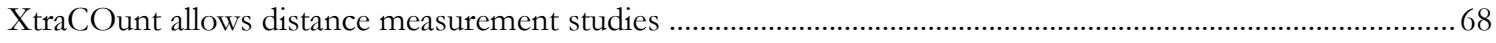

XtraCOunt enhances the speed of rab-10 vesicle distribution studies............................................................. 70

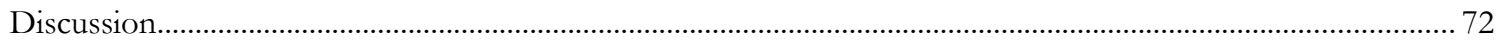


Chapter V. The cuticle of dpy-1 mutant nematodes.....................................................................................75

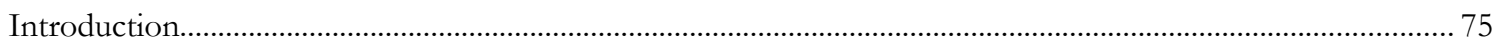

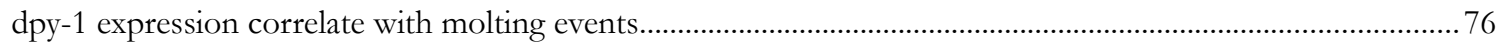

dpy-1 mutant nematodes display cuticle formation defects.................................................................................... 77

Cuticle formation is progressively defective in dpy- 1 mutant nematodes.............................................................8 80

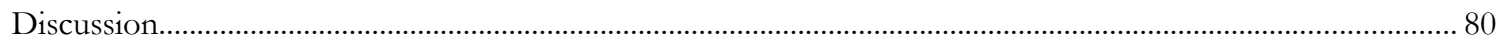

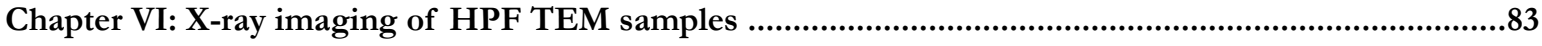

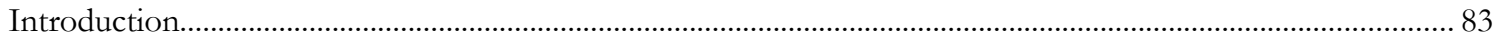

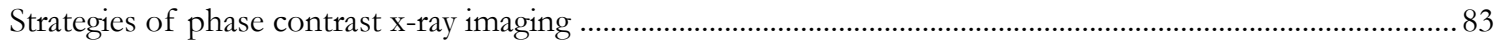

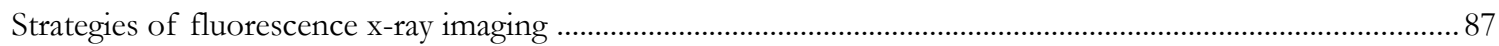

Results from $\mathrm{x}$-ray holography studies of TEM sections...................................................................................... 90

X-ray tomography enables Whole-Worm-Tomography.................................................................................. 94

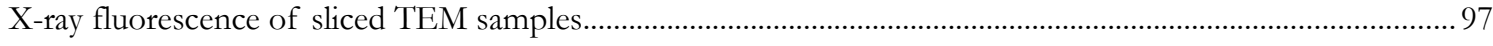

Discussion

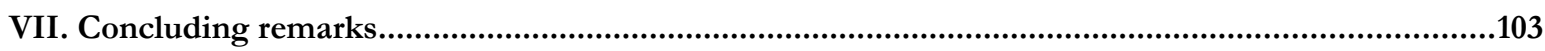

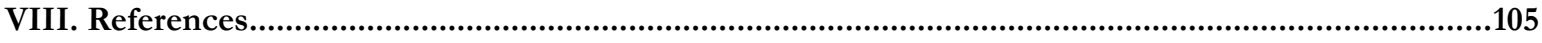



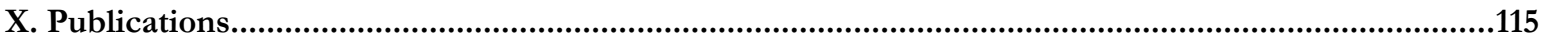




\section{List of figures}

\section{Chapter I}

Figure 1: Intracellular transport uses vesicles as cargo shuttles...........................................................................

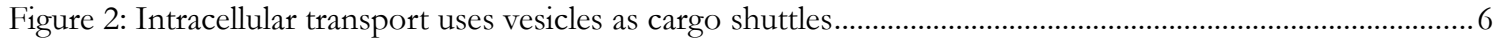

Figure 3: Rab-GTPases associate to intracellular compartments and trafficking routes........................................8

Figure 4: Rab-GTPases act as molecular switches in various steps of vesicle trafficking......................................11

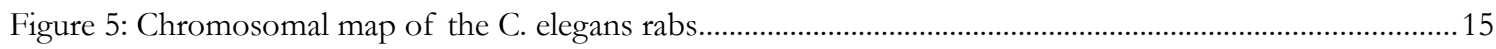

Figure 6 : Stepwise preparation of sample for EM. …………………………………………………………....19

\section{Chapter III}

Figure 7: The exon/intron structure of C. elegans rabs........................................................................................ 30





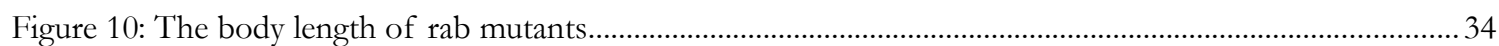

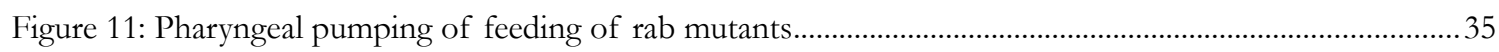

Figure 12: Defecation cycle length and expulsion efficiency of rab mutants..........................................................37

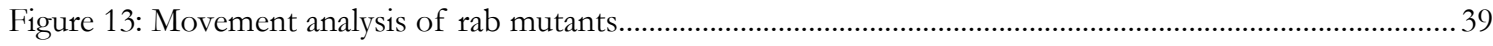

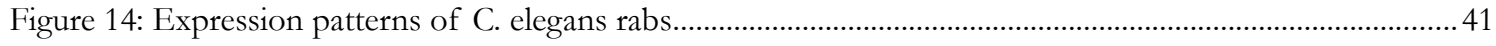

Figure 15: Cross sections through the midbody of the nematode …………………………………………….....42

Figure 16: High resolution TEM tissue catalog ............................................................................................. 43

Figure 17: Serial section and 3D representation of a coelomocyte ....................................................................... 44

Figure 18: Serial section and 3D representations of a synaptic nerve terminal..................................................45

Figure 19: Shape and size of the Golgi in different tissues................................................................................. 46

Figure 20: Electron tomograms and3D model of a Golgi................................................................................... 48

Figure 21: Wiki-Data of rab-8 mutant nematodes.............................................................................................. 50

\section{Chapter IV}

Figure 22: XtraCOunts novel features and versatility ………………………………………………………....60

Figure 23: Individual steps of a vesicle distribution analysis with XtraCOunt. …………………………………...61

Figure 24: XtraCOunt automates the classical analysis procedure. …………………………………………....63

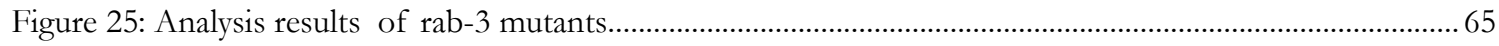

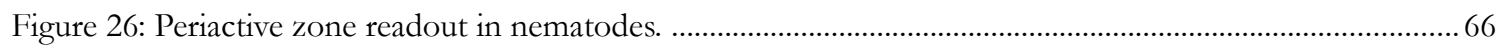


Figure 27: Synaptic vesicle localization in Drosophila. ....................................................................................... 67

Figure 28: Linear distance measurement tool of XtraCOunt. ..................................................................................68

Figure 29: Nonlinear distance measurements with XtraCOunt................................................................................69

Figure 30: Ultrastructual analysis of rab-10 mutant synapses ..............................................................................71

\section{Chapter V}

Figure 31: The growth defect in dumpy worms …………………………………………………………....

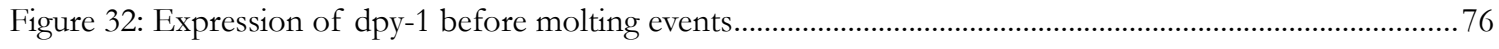

Figure 33: Schematic of the C. elegans cuticle............................................................................................... 76

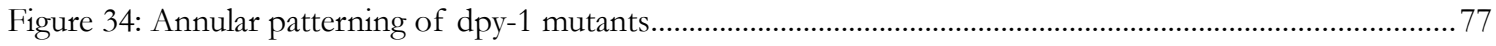

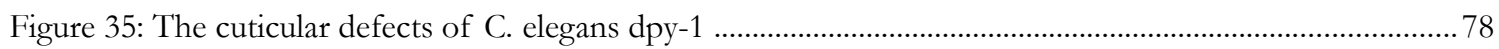

Figure 36: The cuticular defects of P. pacificus dpy-1 ………………………………………………………...... 79

Figure 37: Annular misspatterning progress after molting............................................................................... 80

\section{Chapter VI}

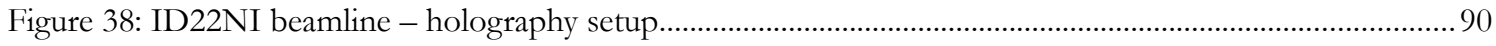

Figure 39: X-ray holography reconstruction of sectioned TEM samples................................................................91

Figure 40: Iterative reconstructions of sectioned TEM samples..........................................................................93

Figure 41: TOMCAT beamline - parallel beam tomography setup....................................................................... 94



Figure 43: Virtual slices of tomographic reconstruction....................................................................................95

Figure 44: Modeling of reconstructed volume visualizes the nematodes tissue anatomy …………..................96

Figure 45: ID21 beamline - microfluorescence setup...................................................................................... 97

Figure 46: Energy spectrum of an ultrathin epon-slice including a nematode cross-section................................97

Figure 47: X-ray fluorescence of a ultrathin epon slice including a nematode cross-section...............................98

Figure 48: Elemental distribution maps of a ultrathin epon slice including a nematode cross-section...............98

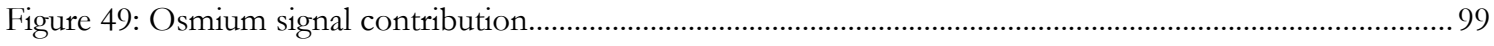




\section{Motivation}

Intracellular transport ensures intracellular communication among all organelles within a cell. Signals as well as materials are shipped via small vesicular carriers. The armada of transport related proteins is orchestrated by only one class of proteins which function as key regulators the family of small Rab-GTPases. These regulators ensure among other tasks the proper filling and budding of vesicles, coordinate the use of unidirectional working motor proteins and finally ensure fusion of the vesicle with their target membrane. They function as molecular switches and are thus able very rapidly to change the activity of their effectors. For a proper understanding of cellular transport processes a higher understanding of the Rab-proteins (RABs) and their cell spanning network is necessary.

The biggest problem in studying intracellular transport processes is resolution. Resolution in terms of its spatial understanding but additionally in terms of its temporal aspect. Up until recently the widely used high-resolution electron microscopy suffered from a rather poorly defined temporal component. Specimen, that were prepared for electron microscopic investigations were fixed with methods that preserved only very small parts of the sample tissue or the preservation procedure took very long - losing the temporal accuracy. In order to capture the intracellular trafficking events in the cell previous fixation methods were of limited use. However, the recent introduction of ultra-rapid high-pressure-freezing method solved this issue and the community is now able to capture and investigate the ultra-fast trafficking events of the intracellular transport machinery.

Using $C$. elegans as a model system, whose transport machinery resembles that of higher organisms and which can now be snap-frozen as a total multicellular organism, a deeper understanding of the network of RABs can be developed. The family of these GTPases is quite small and almost non-redundant in those nematodes. The gained knowledge can later be extrapolated to other more complex model systems were such a broad analysis is not feasible without any a-priori knowledge. Laying the basis for the final understanding of the intracellular trafficking machinery is therefore the ultimate goal of the presented investigations. 
Motivation 


\section{Chapter I. Introduction}

\subsection{Eukaryotes tightly regulate their intracellular transport}

One of the first milestones in the evolution of life was undeniably the encapsulation of life from the outside environment with the help of membranes. Those biological borders protect intracellular content against the extracellular environment and are able to enrich content beyond critical concentrations to facilitate otherwise unfavorable internal reactions (Liposwski and Sackmann, 1995). The introduction of this boundary however raises a very fundamental problem, it requires an effective system that facilitates transport across this membrane. The intracellular transport system of today's eukayotes is able to maintain the integrity and identity of the intracellular organelles and, at the same time, controls crosstalk between intracellular compartments. The transport system furthermore ensures the communication between neighboring cells, which are often highly differentiated (Liposwski and Sackmann, 1995). In order to maintain the desired transport functionality, a highly differentiated machinery has evolved. This machinery maintains organelle identity via a specific transport and recycling system of lipids and proteins. It uses small vesicular carriers that bud off from a donor compartment and it transports these vesicles to their target compartment, where the vesicles ultimately fuse and therby deliver their cargo (Stenmark, 2009). This system shuttles not only the cargo that is present within the lumen of the vesicle but also the lipids and the proteins contained within the lipid bilayer of this vesicle. In order to accurately control this flow of material, precision is needed in both the temporal and in the spacial process regulation (Fukuda, 2008).

The machinery that emerged for this purpose can be separated into roughly three parts. To control vesicle budding and cargo sorting, one part of the trafficking machinery generates a regulatory envelope of coat-proteins around the vesicle (Pucadyil and Schmid, 2009). The second part of the machinery facilitates the fusion event of a vesicle with its specific target membrane. The major contributors for this second system are SNARE proteins (de Wit, 2010). In final control of the spatial and temporal integration of the budding, transport and 
fusion events is the third group of proteins - the RABs. They coordinate all events of the transport processes and are widely accepted to be the key regulators of intracellular trafficking. The RABs belong to the RAS superfamily of small GTP-ases and have been extensively studied in the last years. They are involved in regulation of virtually all intracellular membrane trafficking events (Pfeffer, 2001; Seabra and Coudrier, 2004; Pfeffer, 2007; Pucadyil and Schmid, 2009; Stenmark, 2009).

In the following thesis these governing RABs, are discussed in context of their influence and roles in and on intracellular trafficking. To introduce the proper terminology a short description of the coating machinery and fusion mediating SNAREs is given in advance to display the networks in which RABs are acting. 


\subsection{Fission of membranes is supported and driven by multimolecular coats}

As already postulated by Palade in 1975 (Palade, 1975) the majority of the cell internal vesicular carriers form from coated membrane regions (Stenmark, 2009). In these regions the vesicular carriers bud off from a flat membrane patch with a coat that envelops the entire vesicle on the cytosolic part of the membrane. This coat is shed after budding is complete (see Figure 1).

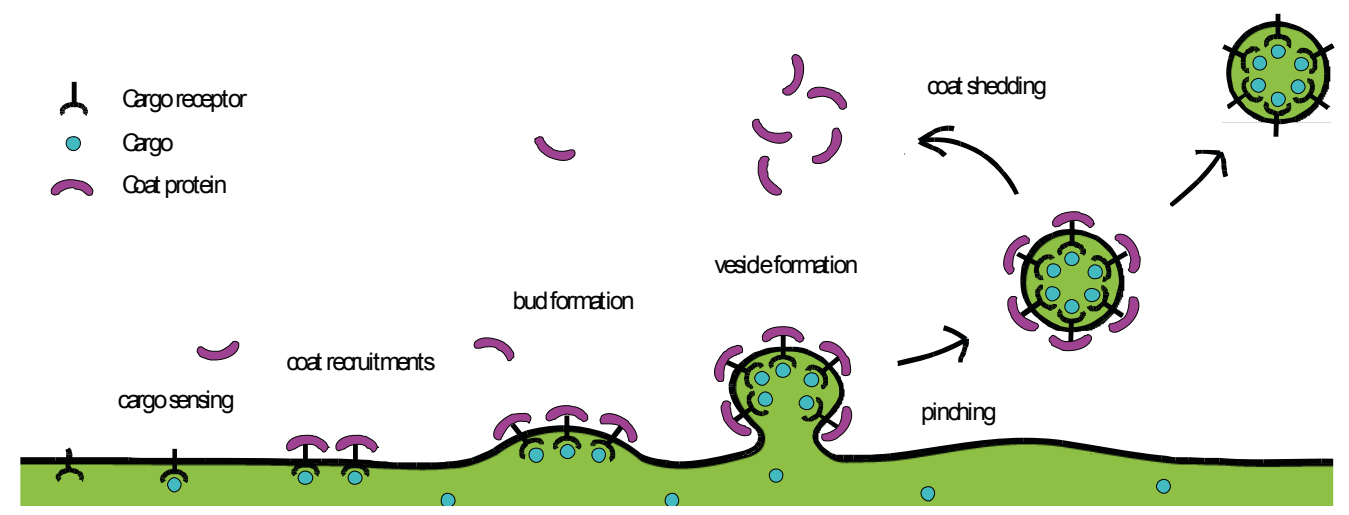

Figure 1: Intracellular transport uses vesicles as cargo shuttles

In the first step of intracellular transport coating proteins stabilize the curvature of newly generated protrusions and help to pinch of vesicles. After budding this coat disassembles from the cargo vesicle.

The coat proteins of the vesicle envelope have two functions. At first the membrane patch, which is covered by the coat, selects and concentrates molecules that are destined for transport and excludes unwanted proteins from the covered area. Secondly, the coat proteins assemble into a basket like envelope that molds and stabilizes the membrane curvature of an emerging bud. This outward protrusion can be easily pinched off. As a result of these two features, the emerging vesicle is loaded only with the appropriate cargo, while at the same time ensuring that it contains all necessary molecules for further processing.

\section{Eukaryotes evolved diverse vesicle coating systems}

In eukoryotes a number of different types of coat proteins have evolved to be used at different stations of the intracellular trafficking routes. The three most extensively studied coat 
proteins are clathrin, coat-protein 1 (COPI) and coat-protein 2 (COPII). Clathrin coated vesicles regulate the exocytosis through the Golgi apparatus and endocytosis from the plasma membrane (Pearse and Bretscher, 1980). The COPI and COPII coated vesicles mainly transport between the endoplasmic reticulum (ER) and the Golgi (Duden et al., 1991; Bednarek, Orci and Schekman, 1996). Less well understood due to their recent discovery are the coats of the retromer complex (Seaman, McCaffery and Emr, 1998) and the ESCRT complex (Katzmann, Babst and Emr, 2001). The retromer complex functions to mediate the retrieval of receptors from the endosome to the trans-Golgi network (TGN) (Seaman, McCaffery and Emr, 1998). The ESCRT complex internalizes membrane proteins at the endosome (Katzmann, Babst and Emr, 2001) to prepare them for degradation in the lysosome.

\subsection{Target-specific fusion of membranes is mediated via SNARE proteins}

To accurately proceed with the membrane traffic newly generated vesicles need to correctly identify the target membrane with which they need to fuse with (Figure 2). Although this process is coordinated by RABs the ultimate fusion event itself is driven by proteins which are collectively termed SNAREs. The soluble N-ethylmaleimide-sensitive factor attachment receptors (SNAREs) represent a highly conserved class of proteins that are comprised of

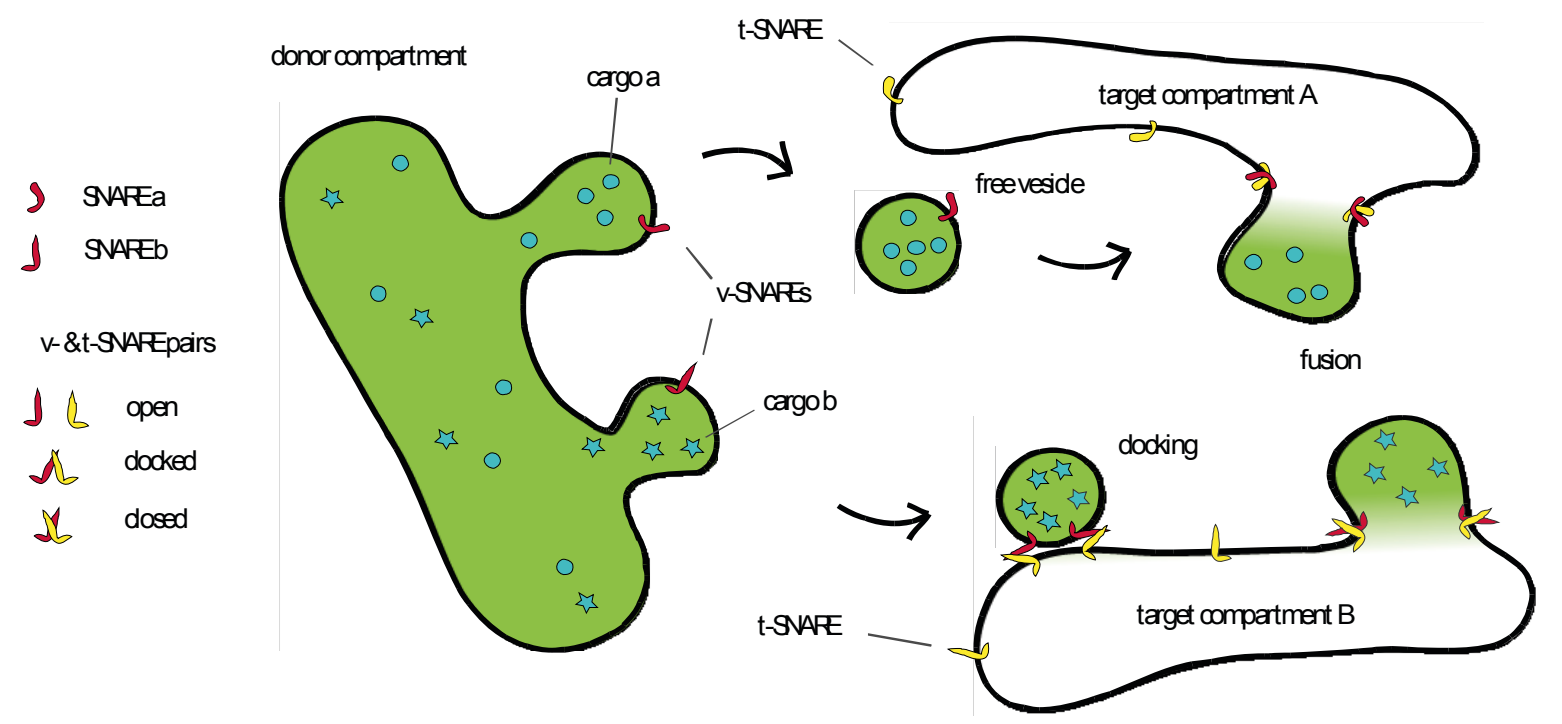

Figure 2: Intracellular transport uses vesicles as cargo shuttles

In the second step of intracellular trafficking free vesicles can be shuttled to their target membrane and undergo docking and subsequent fusion with the help of the appropriate SNARE proteins. 
several domains (Kloepper, Kienle and Fasshauer, 2007). The highly conserved SNARE motif, a sequence of 60-70 amino acids, defines all members of the family. It consists of eight ampiphatic, heptad-repeats which are prone to form coiled coil structures. At the C-terminus the majority of the SNAREs contain a hydrophobic transmembrane region. SNAREs lacking this transmembrane domain instead incorporate hydrophobic post-translational modifications for membrane binding. In order to fuse the vesicular membrane of an incoming vesicle with the target membrane of the acceptor organelle, a set of complementary SNAREs is presented on both the vesicular side and on the target membrane (termed v- and t-SNARE respectively). These SNAREs are able to recognize each other and will selectively bind their cognate partner to form a so-called trans-SNARE complex (Sørensen, 2005). About 35 different compartment specific mammalian SNARES enable large possibilities for very selective transport (Bock et al., 2001). Once a docking is established, the SNAREs undergo a conformational change that triggers the fusion event. The SNAREs anchored in both membranes closely intertwine their helical parts and this pulls the two membranes towards each other, squeezing the water molecules out of the cleft and fusing the membranes. The final result is vesicle cargo delivery into its designated target compartment. Untwisting of the coiled $\mathrm{v}$ - and t-SNARES and subsequent recycling enables SNARE retrieval, allowing for the start of a new round of trafficking. ${ }^{1}$ The molecular basis of the membrane fusion is extensively studied for the specific case of neurotransmitter release, when vesicle exocytosis occurring for the purpose of synaptic transmission events (reviewed in Südhof 2004).

\subsection{Rab-GTPases localize compartment-specific to establish trafficking routes}

The intracellular distribution of Rab-GTPases is very specific. The human genome encodes at least 70 Rab- or Rab-like proteins and all of them localize to specific compartments. Moreover, some of the rabs seem to be tissue specific while others are ubiquitously expressed (Chavrier, 1990; Zerial and McBridge, 2001).

1 For the rather functional notation of $\mathrm{v}$ - and t-SNAREs a renaming to R- and Q-SNARES has been suggested (Fasshauer et al., 1998; Kloepper, Kienle and Fasshauer, 2007). This notion is based on the amino acid residue that these SNAREs contribute in a conserved fashion within the twisted coiled coil (in the ionic 'layer 0 '). The notation, despite of the higher accuracy on the molecular level, will be neglected here to allow a easier association to the trafficking context. 
A general localization of the Rabs is illustrated below (Figure 3). Their detailed roles at their localization have been recently reviewed by Harald Stenmark (Stenmark, 2009).

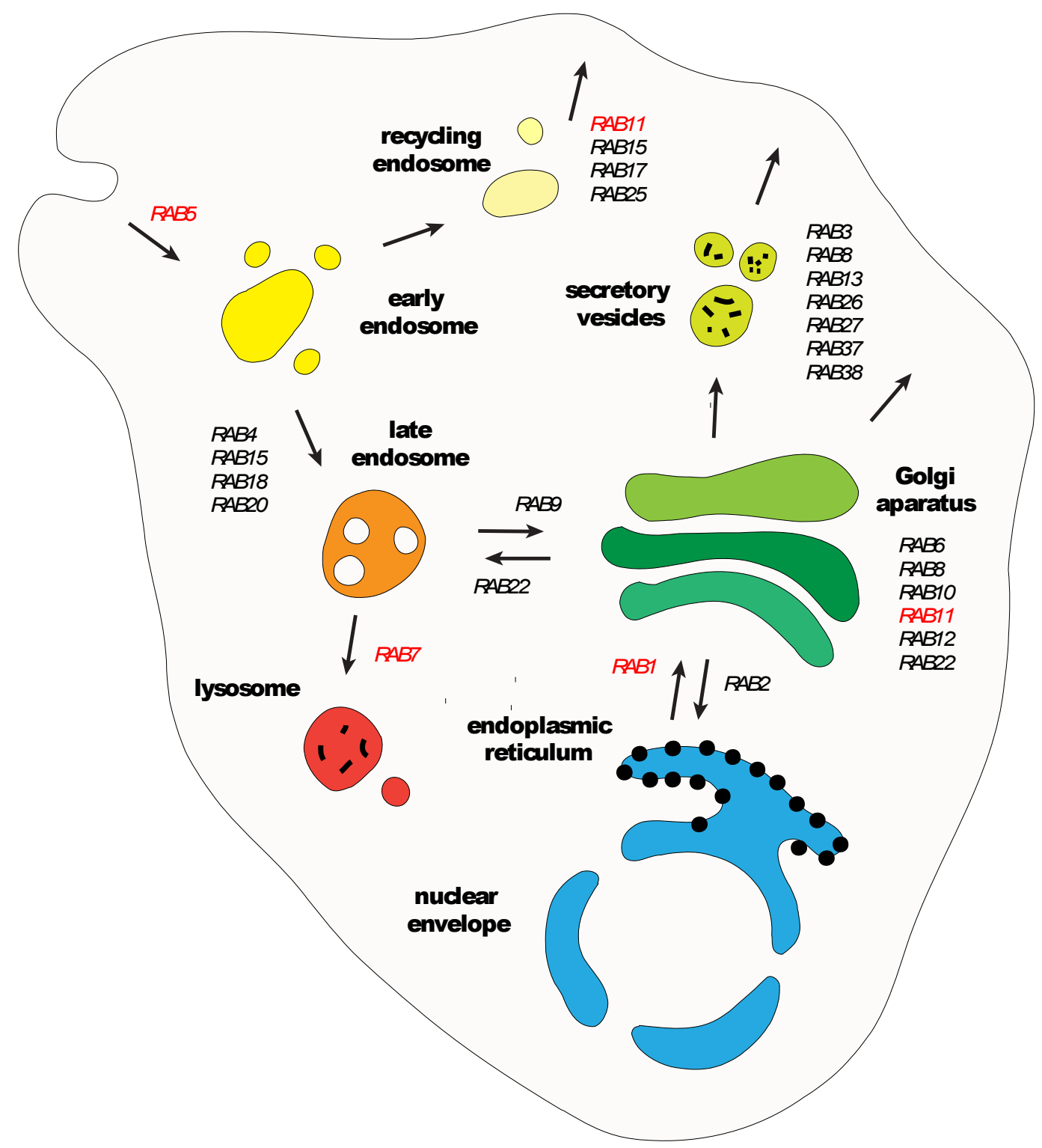

Figure 3: Rab-GTPases associate to intracellular compartments and trafficking routes.

The scheme summarizes the localization of RABs to intracellular organelles in the mammalian system. Canonical RABs - which lead to death upon deletion - are shown in red. Major trafficking routes are indicated with pointing arrows. In case a RAB can be directly linked to these routes they are indicated next to the arrows. 


\section{GDIs and GDFs position Rab GTPases}

To transport Rab-GTPases to their specific compartments a unique functional system evolved. The mechanics of this system where well-summarized recently (Goody, Rak and Alexandrov, 2005). In brief, Rabs are delivered to their destined membranes via GTPase dissociation inhibitors (GDIs). The GDIs bind to the geranylated, inactive Rabs and stabilize this form. They were originally thought to solely prevent the release of GDP from the Rabs (Matsui et al., 1990) but later studies revealed their additional roles in chaperoning, recycling and transport (Ulrich et al., 1993; Soldati et al., 1994). If the Rab is newly synthesized and not yet geranylated, the Rab escort protein (REP) is required to present the Rab to the geranylgeranyl-transferase (GGT) before the transport to the target can be initiated (Alexandrov et al., 1994; Seabra, 1996). The Rab-GDI complexes are recognized by membrane-bound GDIdisplacement factors (GDFs). These GDFs function to displace the Rabs from their GDI, in order to facilitate the release of the Rab to their destined membrane (Sivars, Aivazian and Pfeffer, 2003). Once the Rab is integrated, it can diffuse through the plane of the membrane and there it can be activated to fulfill its function. This key feature of the Rabs - a cycle between inactivation and activation - and the effect of Rabs on specific downstream effectors in intracellular trafficking events will be discussed in the course of this chapter.

\section{Initial Rab transport is not fully understood}

Noteworthy is that the localization of the Rabs is firstly governed by the GDI/DGF system however the DGFs are shuttled via Rab regulated transport events. Thus a question is raised: how does the Rab initially get to its target membrane? Such de-novo transport is never necessary since Rabs are always present in the cells. Previously as maternal effect, later though self generated supply. However, it is still an interesting issue and is currently envisioned to happen by hypothesized key effectors, which help to stabilize the Rabs in microdomains. However, issue far is from being understood and remains focus of current research (Pfeffer, 2005). 


\subsection{Rab-GTPases orchestrate trafficking through active recruitment of effectors}

The small Rab-GTPases have been found to be central regulators of vesicle budding, motility and fusion (Zerial and MCBride, 2001; Pfeffer, 2005; Stenmark, 2009). The regulatory GTPases can be considered to be molecular switches that are able to cycle between their 'active' GTP-bound (guanosine-triphosphate) form and their 'inactive' GDP-bound (guanosine-diphosphate) form. Their ability to rapidly change their effectors activity, together with their specific localization to individual intracellular compartments, allows the very precise spatio-temporal action that is needed for the proper regulation of the cellular trafficking network.

\section{Rab proteins cycle between their active and inactive state}

The cycling between the GDP- and GTP-bound form of the Rabs (Figure 4A) is catalyzed by guanine nucleotide exchange factors (GEFs). These GEFs function to recognize specific variable 'switch' regions in the Rabs in order to facilitate a conformational change of these regions, allowing GDP release from the Rab (Delprato, Merithew and Lambright, 2004). This GDP is then immediately replaced by GTP due to the naturally high cytosolic GTP concentration. In this GTP-bound form, the Rab is able to bind and associate with various effectors with their switch and interswitch regions, as it is discussed in the next section. To complete the regulatory cycle, the GTP needs to be converted to GDP, thereby releasing inorganic phosphate. Although the Rabs have an intrinsic constitutive but slow GTPase activity the hydrolysis from GTP to GDP is virtually always assisted by a GTPases activating protein (GAP). This GAP assists catalysis by providing an alanine- and a glutamine residue to accelerate the GTP conversion (Pan et al., 2006).

\section{Rab effectors influence a multitude of trafficking steps}

Evidence for the effector recruiting influence of activated (GTP-bound) Rabs can be found in virtually any step of vesicular transport. These effectors can be categorized into 5 groups according to the sequential role during the trafficking process (Figure 4B). 
A

GDI - GDPdissociation inhibitor shuttling \& recycling

GDF- GDI displacment factor

GF-Guanosine exchange factor

GAP-GTPase activating protein

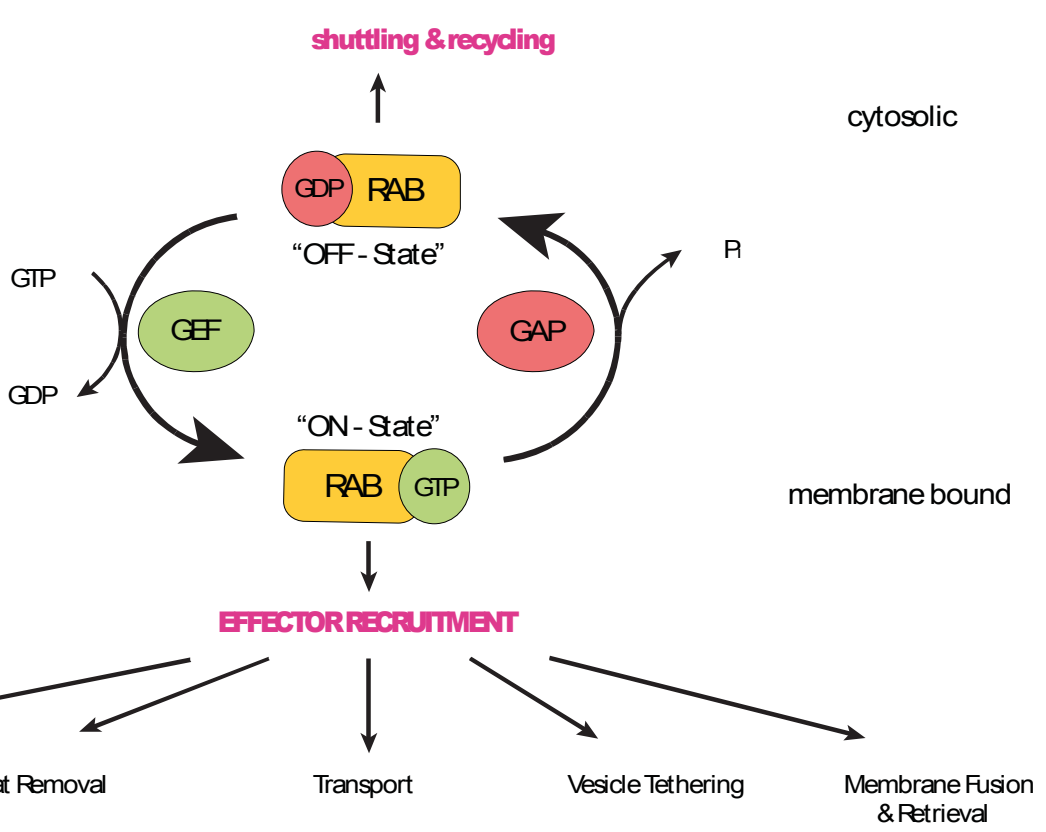

B

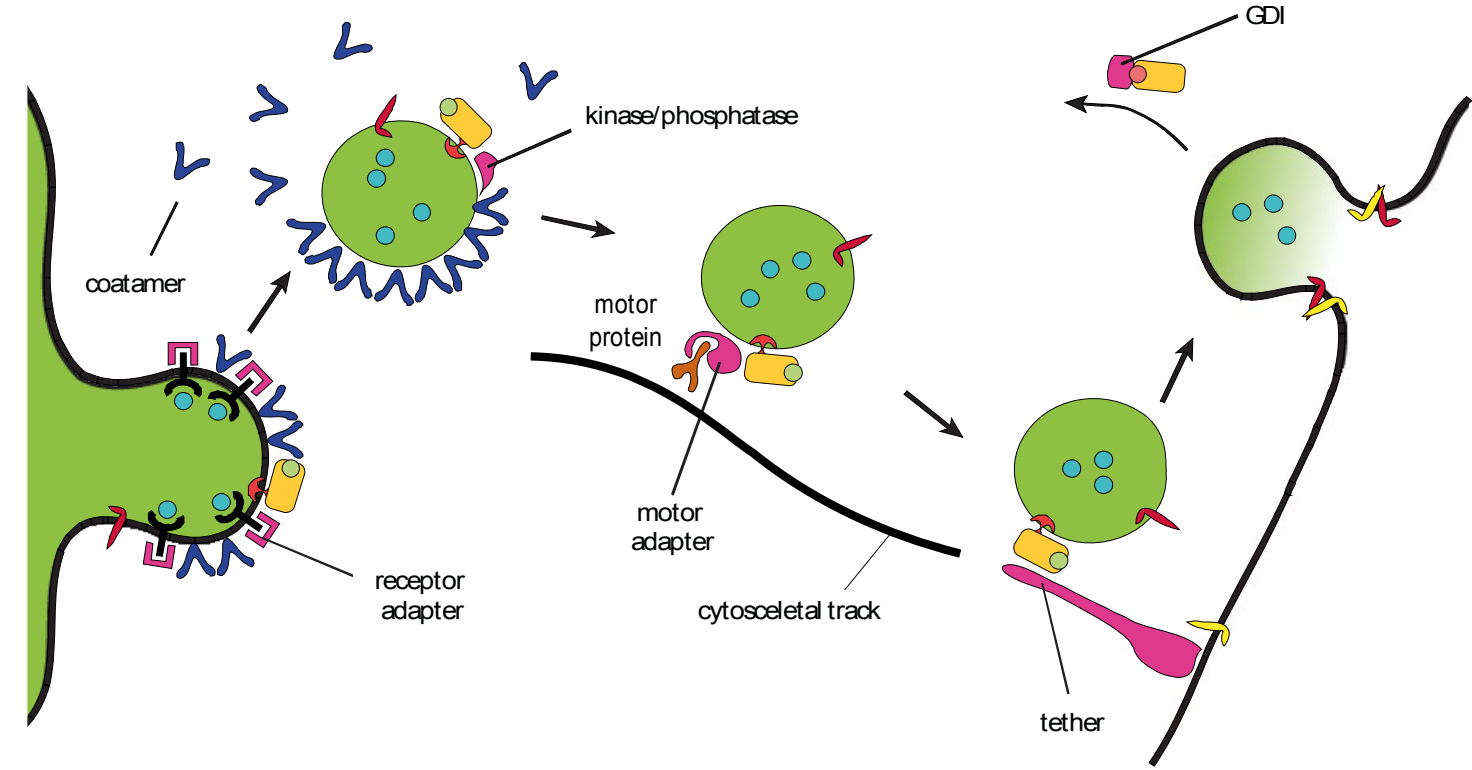

Figure 4: Rab-GTPases act as molecular switches in various steps of vesicle trafficking

A) The Rab-cycling between the GDP- and GTP-bound form is facilitated by GEFs and GAPs. The Rabs are shuttled to their target membrane via the GDI/GDF system. B) Integrated in their target membrane, upon activation, Rabs associate to various types of effectors that function to initiate a crucial step during trafficking.

\section{Rab effectors influence cargo-sorting and coat assembly}

Before a new vesicle is generated the maintenance of the trafficking system is already active to sort cargo material into the future vesicle. As an example, Rab-5 together with GDI, have 
been shown to facilitate the sequestration of ligands into clathrin-coated pits during clathrin mediated endocytosis of tranferrin (McLauchlan et al., 1998). Rab-9, as a second example, has been shown to enhance the sorting specificity for the mannose-6-phosphate receptors (M6PRs) in late endosomes via the recruitment of the sorting adaptor TIP47 (Carroll et al., 2001).

\section{Vesicles shed their coat after budding through the help of Rab-effectors}

Once the nascent vesicle detaches from the donor membrane the coat-proteins must be shed from the vesicles as they would interfere during fusion. This shedding is again regulated by Rabs. In the case of clathrin coated vesicles, Rab-5 together with its GEF and the VPS9 containing protein 1 (GAPvD1) promote coat shedding (Semerdjieva et al., 2008). The AP2associated-kinase-1 (AAK1) usually enhances the binding of AP2 to phosphatidylinositol-4,5bisphosphate (PtdIns(4,5)P2) by $\mu 2$-subunit phosphorylation. This AAK1 is displaced by the GAPvD1, leading to the dephosphorylation of $\mu 2$. The additional activation of the PI-3kinase and PI-phosphatase then lead to the increased turnover of PtdIns(4,5)P2 (Christoforidis et al., 1999; Shin et al., 2005). This dual action then destabilizes the adhesion of the AP2 coatamers. All effects in combination ultimately lead to the shedding of the vesicle coat.

\section{Motility of vesicles is directly and indirectly mediated via Rab-effectors}

Polar cytoskeletal tracks are made of cell spanning microtubules, while local networks consists of actin filaments. Motor proteins are able to attach to these proteins and are able move on these fibers in a uni-directional fashion.

Microtubule dependent traffic in the direction of the cell periphery is mediated by kinesins whereas transport towards the inner cell is governed by dyneins. Both of these motor families are regulated by Rab-GTPases. Kinesins can be direct effectors of Rabs, such as the Golgi associated Rabkinesin 6 (KIF20A) by Rab-6 (Echard et al., 1998) or indirect, such as KIF16B, which can bind to a Rab-5 controlled conformation of PtdIns-3-phosphates at endosomes (Hoepfner et al., 2005). Dyneins have not been shown to interact directly with Rabs but they are however indirectly regulated. Indirectly regulated proteins include the Rab-interacting lysosomal protein - a late-endosomal Rab-7 effector (Jordens et al., 2005) or the Rab-6 effector bicaudal D1, which mediates attachment of Golgi vesicles that are prone for transport 
to the ER (Matanis et al., 2002).

Similar to the microtubule-dependent motors, the activity of the actin-dependent motors (myosins) is also Rab-regulated. The class 5 Myosins, as an example, transport towards the cell periphery. Myosin 5A transports melanosomes to the cell periphery of melanocytes via Rab27A-regulated melanophilin adaptors. In this context, mutation of Rab27A has been directly linked to the causes of the Griscelli syndrome (Menasche et al., 2000).

Thus, the Rab-GTPases interact through their effectors with a vast range of motors. They play an important role in the proper attachment of vesicles to the motors that bring these vesicles to their destined target for both long and short range transport.

\section{The role of Rab-GTPases in vesicle tethering}

The fourth step in vesicle-mediated membrane traffic is called tethering. Tethering is a term used to describe the initial interaction between a vesicle and its target membrane. The tethering process precedes the pairing of SNAREs on opposing membranes. The pairing of these SNAREs was initially postulated to drive the specificity of vesicle targeting (Söllner et al., 1993). However, more recently it has become clear that tethers or tethering complexes mediate the initial contact of the vesicle and the membrane (Kummel and Heinemann, 2008). Currently two groups of tethering factors have been identified: large multi-subunit complexes and long coiled-coil proteins (Cai, Reinisch, and Ferro-Novick, 2007).

For the first group, as many as eight conserved complexes acting in exocytic and endocytic trafficking events have been identified to date. Five of them are involved in secretion: TRAPPI (ER-Golgi), TRAPPII (intra-Golgi/endosome-late Golgi), Dsl1 (Golgi-ER), COG (endosomeearly Golgi) and the exocyst (Golgi-plasma membrane; endosome-plasma membrane) (Andag et al., 2001; Cai et al., 2005; Munson and Novick, 2006; Reilly et al., 2001; TerBush et al., 1996; Whyte and Munro, 2001). The other three are required in sorting events: CORVET (late Golgiendosome), HOPS (endosome-vacuole) and GARP/VFT (endosomelate Golgi) (Peterson and Emr, 2001; Conibear et al., 2003; Peplowska et al., 2007).

The long coiled-coil proteins - as the second group of tethers - have been hypothesized to function as anchored fishing ropes. These proteins, often spanning across lengths bigger than entire vesicles are thought to reach out for incoming cargo-vesicles while bound to the target membrane. Classical examples for this group of tethers are the golgins, the early endosomal autoantigen EEA1 and Rabenosyn 5 (Sonnichsen et al., 1998; Simonsen et al., 1999; Barr and 
Short, 2003). Among these tethers severall have been shown to be under the control of Rabs (reviewed in Grosshans, Ortiz and Novick, 2006) and some Rabs even act in multiple stages of the endo- and exocytic pathways. Rab-1, for example, has been shown to be required for ERto-Golgi- and intra-Golgi traffic as well ass membrane recycling by the early endosome (Baker et al., 1990; Davidson and Balch, 1993; Jedd et al., 1995; Lafourcade et al., 2004).

\section{SNARE function during vesicle fusion is influenced by Rab-GTPases}

SNARE proteins are the key players for the fifth and final step of the vesicle cycle, that beeing vesicle fusion. However, even at this last step, the governing influences of Rab-GTPases are traceable because many SNAREs need to physically interact with previously Rab-dependent tethering complexes. Tethers act by pulling the vesicles closer in order to increase probability of $\mathrm{v}$ - and t-SNARE interaction and moreover they can actively promote generation of the trans-SNARE complex. As an example, the coiled-coil tether p115, a Rab-1 effector, functions in this dual fashion on COPII coated vesicles in ER-Golgi transport(Bentley, 2006).

\subsection{Characterizing the entire Rab-family}

As discussed above, Rab-GTPases function as central regulators of the intracellular trafficking networks in eukaryotes, a topic widely studied in recent years (Pfeffer, 2001; Zerial and McBride, 2001; Pfeffer, 2007; Stenmark, 2009). However, despite the fact that various studies utilizing a plethora of model systems (both in-vivo and in-vitro) have been performed, it still remains difficult to compare the emerging and sometimes contradictory results from different laboratories, different techniques and various equipments. For this reason, my thesis aims to generate a thorough analysis of all Rab-GTPases in a single experimental system. For severall reasons the nematode Caenorbabditis elegans was choosen as a suitable model system. Firstly, of all the nematode can be maintained and easily cultivated in the laboratory. Beyond its affordable and effective maintenance, the worm offers unique feature making it an ideal tool for investigations. Two of the most obvious, but at the same time essential, features is that the worm remains translucent throughout its life-cycle and it can be easily genetically modified. This renders the worm suitable for light- and fluorescent microscopy in combination with genetic engineering techniques. Additionally, the entire organism has been mapped on a cellular 
level (Riddle et al., 1997). Both the availability of good imaging techniques as well as comprehensive anatomical knowledge are of essential importance to the morphologic and genetic characterization approaches used in this study.

\section{The nematode genome is completely deciphered}

C. elegans was the first multicellular organism to have its genome completely sequenced (C. elegans Sequencing Consortium, 1998, Hillier et al., 2005). The genome sequence is approximately 100 million base pairs long and contains about 20000 protein-coding genes distributed on six chromosomes. About 30 of these genes belong to the family of small RabGTPases in C. elegans. The nematode therefore shares a rab complexity comparable to the human or mouse system but with reduced genetic redundancy (Pereira-Leal and Seabra, 2001). Due to the existence of the well curated databases for the C. elegans genome it was possible to locate the sequences of all rabs very accurately within the nematode genome as found in a preliminary analysis (Figure 5). The distribution is fairly random, with chromosome five carrying only one of the rabs.

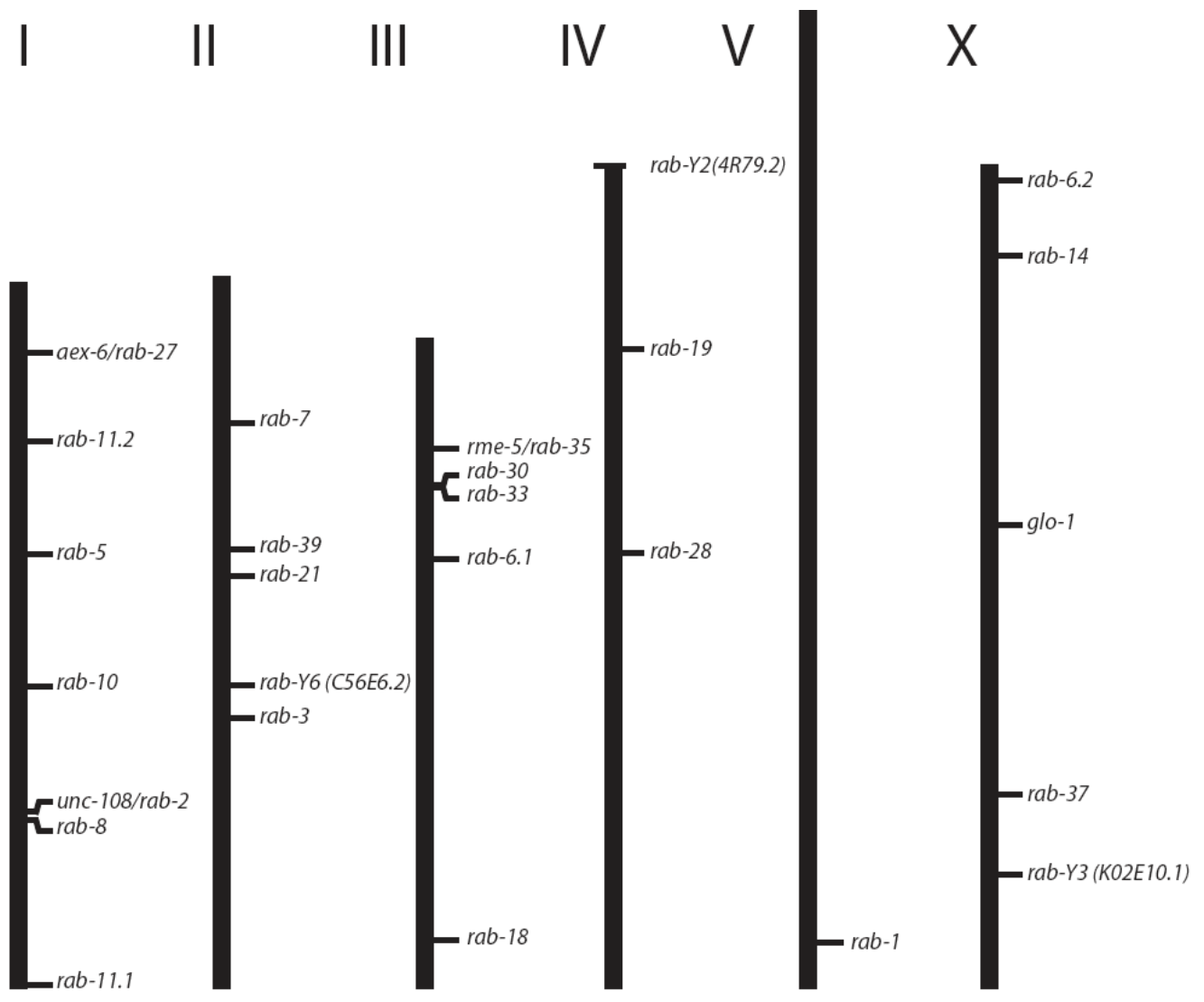

Figure 5: Chromosomal map of the C. elegans rabs

Rab locate on all of the chromosomes (linkage groups) of the C. elegans genome. 


\section{A Rab-Atlas will describe the entire family of Rab-GTPases}

The Eimer laboratory is working on a description for the entire rab family of the worm. This project will upon completion reveal the Rab-GTPases individual involvement in the worm trafficking network. While this group project is driven by all members of the Eimer-lab, my part of this study was the investigations on an ultrastructural level. In a first step, my project intended to describe the behavioral phenotypes of the worms and aims to generate a morphological description of the cellular- and sub-cellular anatomy of the worm. In the second step, mutants of all available rab family members are characterized in the same fashion and subsequently compared.

The generated morphological atlas - describing wild type and mutants - is intended to display high resolution representations of the complete tissue-, cellular and sub-cellular anatomy of the worm. Additionally the atlas will contain the results from the behavioral tests - which characterize motor programs of locomotion, defecation and feeding. These combined, current results represent an initial platform for further studies on molecular levels, that other group members will then concentrate on.

\subsection{The craft of preserving biological specimen for electron microscopy}

From microscopy studies with visible light and fluorescence techniques our understanding of cellular anatomy has been improved and has lead to the current understanding of the dynamics of cellular processes. Despite these advances the investigations with light microscopy are intrinsically limited to a resolution of down to $200 \mathrm{~nm}^{2}$. However to fully understand the nature of most cellular processes, one needs a resolution capable of resolving the ultrastructure of cells. Thus this study uses electron microscopy (EM), which allows for the visualization of structures within the nanometer range and thereby enables more accurate studies. Due to the fact that electron microscopy samples have to be observed under high vacuum and furthermore have to withstand the effects from electron beam radiation, special

2 Recent advances in light microscopy have shown to overcome this barrier. Techniques such as, STED, STORM, PALM or TIRF microscopy have lowered the given resolution limit by clever designed optics and ingenious approaches but will in the end face a final limit which is still far from the resolution which can be achieved imaging with electrons. As these techniques can be used for living systems they are however of fundamental importance to shift the boundaries of our understanding of cell architecture, structure and function. 
techniques of preservation were developed. However, as an electron beam is heavily absorbed by matter, it is therefore not able to penetrate through a thick specimen ${ }^{3}$. Thus, specimen samples are embedded in plastic and cut into very thin sections. These sections can then be imaged. In order to enhance the contrast of the biological samples in these sections, additional staining- or labeling procedures with heavy metals such as osmium, lead or uranium are typically utilized. The full process of the preservation, embedding and staining of biological specimen is presented in more detail below.

\section{Slow diffusion impairs the results of conventional sample preservation techniques}

Techniques designed to enhance the preservation of biological material have been developed for usage in a variety of scenarios, whether it be foodstuff preservation, specimen conservation from expeditions or even sacred burials found in ancient cultures. Up until recently, scientists believed that ultra-rapid freezing of specimen usually led to the best results - especially in the case of sample preparation for electron microscopy (McDonals and Auer, 2006). Conventional preservation methods for biological samples typically involve processing with a series of chemicals that must penetrate the entire sample via passive diffusion. However, intrinsic to the basic properties of biological samples is that they contain built-in diffusion barriers. Thus, chemical infiltration using passive diffusion methods is relatively slow and by the time the fixative reaches all desired structures, the cells are likely to have altered due to the consequences of cell death. Furthermore, most of the typically used fixatives are selective crosslinkers, which allows for washout effects of non-fixed molecules. Also, as most of the conventional methods use a dehydration step in their protocols, the remaining material is often moved within the cell during the dehydrative, deformative processes. The original cell architecture is therefore often distorted during these procedures. The classical methods here described are therefore not usable in nematodes, because the nematodes cuticle is a very effective diffusion barrier. Relying only on passive diffusion, it would take several hours for chemical penetration of the worm and thus many cellular components would have be degraded or moved away from their original location before fixative could reach them. Thus, fixation artifacts and the time-frame for fixation with these classical methods do not allow us to describe intracellular trafficking events within the worm with the desired accuracy. Thus, for

3 A more comprehensive explanation for the underlying absorption effects can be found in introduction to $\mathrm{x}$-ray chapter VII. 
nematode fixation, opening the worm with a blade or 'breaking' it into two pieces was considered to the best way to achieve fixation. However, these tricks cannot sufficiently compensate for the slow fixation that occurs within nematodes, such that preservation of the tissues is still unsatisfactory.

\section{Cryo-immobilisation can vitrify materials within milliseconds}

In contrast to the conventional methods, ultra-rapid freezing of the specimen allows a instantaneous cryo-immobilisation of all molecules within milliseconds. The extremely fast nature of this process minimizes the ice crystal formation. If ice crystals were to form, the cytoplasm would be distorted by the expansion of the crystals. Biological structures would therefore be excluded from the ice and then moved along with the advancing liquid-solid interface (McIntosh, Nicastro and Mastronarde, 2005). However, if the cooling rate is fast enough to prevent the growth of ice crystals the water molecules will form a so called amorphous, glass-like state (also referred to as non-crystalline, frozen-hydrated or vitreous). To therefore cool a sample with the desired speed, a small sample can be snap-frozen by for example, plunging it into liquid ethane, which has a cooling rate of $10000^{\circ} \mathrm{C} / \mathrm{s}$ (Sosa et al., 1994) or by slamming the sample onto a previously cooled metal block (Sitte, Edelmann and Neumann, 1987). For both methods plunge or impact freezing, several micrometers of the sample will be accurately frozen but beyond that ice-crystals will destroy the sample. This dimension is large enough for most cells and in the case of very large cell cryoprotectans such as sugar or glycerol can be used. In general these protectants are however avoided, if possible because of their "unnatural" influence on the cell structures (Dubochet et al., 1988).

\section{Sample size limitations in rapid freezing can be boosted by application of high- pressure}

Freeze plunging and impact freezing were the most used techniques until Hans Moor developed the idea to use high-pressure to slow down the nucleation and growth of ice crystals. By keeping the sample chamber under high pressure (2000 bar) the rapid freezing of 
samples as large as $0.5 \mathrm{~mm}$ was achieved while erasing the contamination with ice crystals (Moor and Riehle, 1968; Gilkey and Staehelin, 1986).

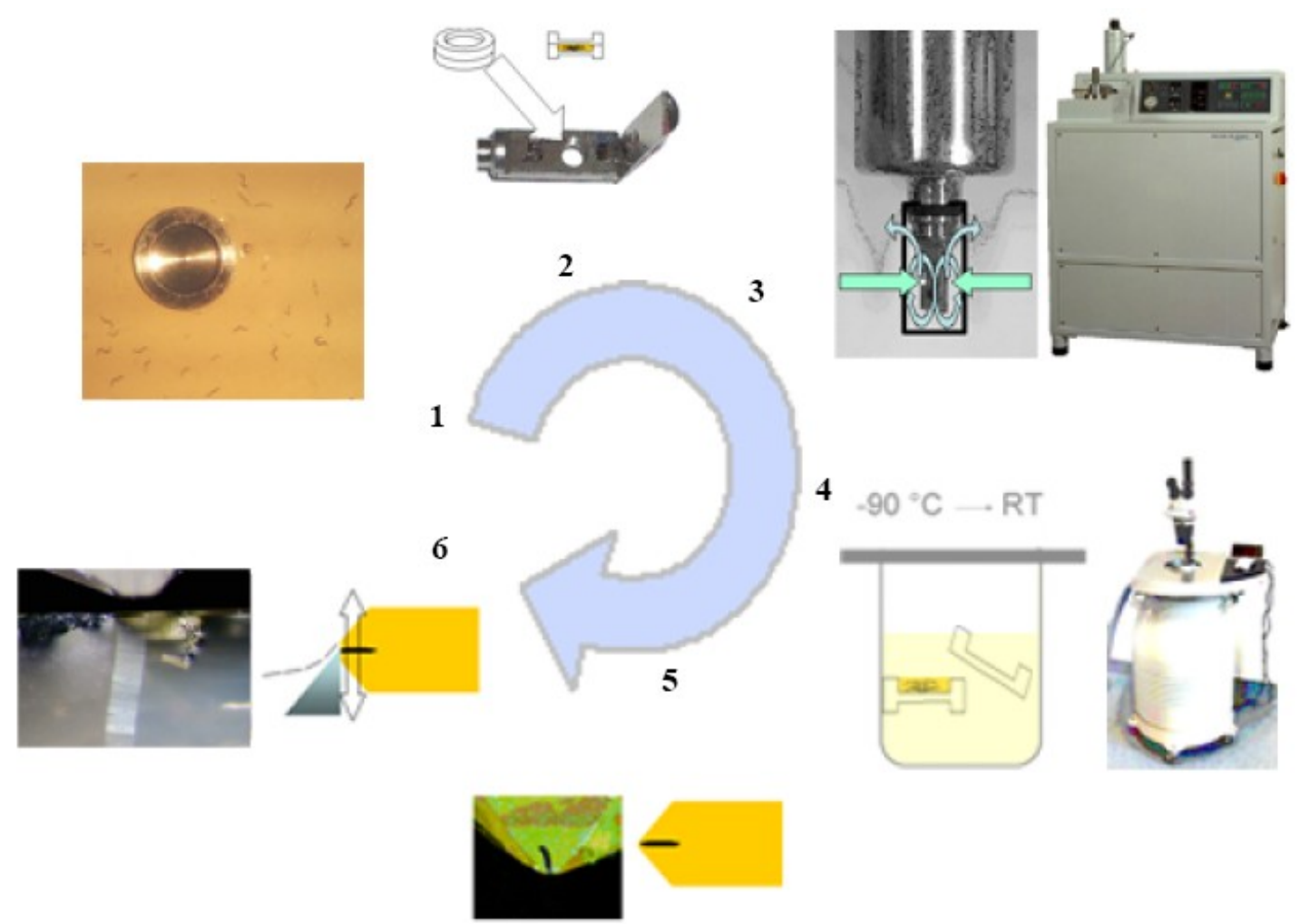

Figure 6: Stepwise preparation of sample for EM.

Up to 20 worms are picked and collected in a specimen carrier (1) filled with E. coli paste. The carrier is closed by a lid, without including air in the interior. A $100 \mu \mathrm{m}$ layer of E. coli paste containing living worms is enclosed between two metal plates. This so called "sandwich" is mounted in a bracket (2) and brought into a pressurechamber which is filled with alcohol at low pressure, followed by a high pressured ,shot" of liquid nitrogen, that expels the alcohol through a valve (3). By this procedure the specimen is already under high pressure just before it is cooled down rapidly. The frozen sandwich is cryo-transferred into a cooling chamber (4), opened and soaked with organic solvent, containing variable contrasting agents like osmium tetraoxyde, uranyl acetate, tannic acid. Osmium tetraoxyde causes optimal membrane contrast, but has to be omitted for immunolocalization. The vitrified water gets exchanged against the solvent during several days, while the contrasting agents penetrate the tissue. Finally the temperature is then increased to RT. Thus the samples become completely infiltrated with liquid resin which can thus be polymerized en-block (5). The blocks can be stored easily for long periods of time. Using a diamond knife ultra thin sections can be cut off the resin, including slices of the embedded worm (6). For more details the reader can refer to Rostaing et al.,2006.

This dimension allows the freezing of small specimen containers, filled with up to 20 adult worms simultaneously, and preserves these worms in a vitreous state as a whole organism. A schematic work flow is depicted above (Figure 6). Only very rapid freezing $\left(0^{\circ} \mathrm{C}\right.$ to $-100^{\circ} \mathrm{C}$ in $10 \mathrm{~ms}$ ) allows the capture of fast trafficking events such as the fusion of membranes, a feature important to the study of ultra-fast synaptic trafficking events in neurons. 


\section{Freeze substitution protocols allow for morphologic or immunolabeling studies}

After cryoimmobilisation the actual crosslinking of all material is performed by substituting an organic solvent for the water in the specimen at low temperatures. The process has been termed freeze substitution, as it is carried out at temperatures from about $-78^{\circ}$ to $-90^{\circ} \mathrm{C}$ (McIntosh, Nicastro and Mastronarde, 2005). In most cases, this solvent is liquid acetone, which will replace the water content of the specimen in a very gentle molecule by molecule fashion. As temperature is slowly raised the actual fixation is then completed by fixatives such as glutaraldehyde or osmium tetroxide. Their fixation is carried out at about at $-50^{\circ}$ and $-30^{\circ} \mathrm{C}$ respectively, a temperature where the proteinacious and lipid content of the samples is still completely immobilized. As temperature rises to a temperature where the intracellular material could move the specimen is already fixed. A final embedding is then carried out by replacing the organic solvent with either hard epoxy resins for a good morphologic preservation or methacrylate resins for immunolabeling studies (Litwin, 1985). These resins are able to maintain the antigenicity of the specimen but are a slightly softer. The resins are subsequently cured by heat or UV radiation and the resulting plastic blocks can then be cut into ultra-thin sections with a diamond knife.

The wild type and mutant worms embedded for the the Rab-Atlas study were all embedded in hard epoxy resins to firstly preserve the ultrastructure in the best fashion. Moreover, the hard epoxy resin allows for accurately and repetitively slicing of ultra-thin section in the range of 30 to $50 \mathrm{~nm}$ needed for the present studies. ${ }^{4}$

\subsection{Project description}

In order to generate a high resolution anatomic nematode description, the technique of highpressure freezing was combined with a low-temperature fixation to prepare entire nematodes for electron microscopy imaging. This preservation technique is currently the most advanced method available and allows for a high sample throughput. In a first step, wild type worms

4 It is possible to image the frozen-hydrated sections directly. This is considered to be the most native condition a sample could possible be imaged in, but this imaging requires a full work-flow at cryo-temperatures in combination with a cryo-electron-microscope. Furthermore the contrast for this technique is very weak while the applicable dosage is fairly low. Both problems can be overcome but raise the cryogenic imaging to complete craft that is unfortunately not suited for high throughput imaging attempts. These techniques are thus neglected here but they are well described in a recent review by Wiebke Möbius (Möbius, 2009). 
were imaged, and nematode tissues were categorized with a special focus placed on the most central trafficking organelle, the Golgi-apparatus and on the synaptic trafficking in neurons. Secondly, to gain insight into the individual roles of RABS, wildtype tissue results were compared to the tissues of the individual rab mutants.

To aid the evaluation of microscopic data (mostly originating from electron microscopic studies), a novel image analysis tool, XtraCOunt, was developed. The tool is designed for investigating synaptic features and during these studies it helped to characterize synaptic features of multiple rab mutants. Additionally, side projects of specific trafficking problems were investigated. The presented project describes $d p y-1$. Mutations in $d p y-1$ lead to alterations in the worm cuticle, which make the cuticle less flexible and ultimately lead to a dumpy worm phenotype. The understanding and description of this mutant worm strains was possible with the help of XtraCOunt and HPF-EM.

To conclude these studies, the possibilities of $\mathrm{x}$-ray imaging of the TEM samples were investigated. The methods of the $\mathrm{X}$-ray community have proven to be a promising technique for future correlative imaging studies.

\subsection{Structure of the thesis}

In the following result chapters, initial characterization from the rab analysis are presented together with the generated morphological atlas and its Wiki platform (chapter III). Secondly, the novel image analysis tool XtraCOunt, created for the analysis of neuronal trafficking during the Rab-study, is presented in chapter IV.

In three further chapters, the potential of the technique of high-pressure freezing is further explored. Two of these chapters additionally include use of the XtraCOunt imaging tool and one chapter attempts to overcome the limitations of classical EM studies with the help of Xrays.

Phenotypic aberrations of the nematode cuticle by mutations in $d p y-1$ are shown in chapter V. This, thanks to this study, finally classified mutant is the first ever described mutant nematode by Sydney Brenner - the godfather of C. elegans research. Here a first mechanistic explanation 
of this mutants phenotype is provided.

Finally, the preservation by high-pressure freezing was tested as an embedding for biological specimen in $\mathrm{x}$-ray imaging attempts. High resolution 2D-phase-contrast imaging, 3D holotomography and micros-fluorescence studies with embedded worms was performed. The results of these $\mathrm{x}$-ray studies are presented in chapter VI.

Due to individuality of each result chapter and the various aspects addressed within them, the obtained results of all studies are individually discussed in their own chapters for reasons of clarity. Furthermore, in order to be more concise, each sub-project is introduced briefly by itself. Each of the following chapters therefore exists as a single unit, that can be read and understood without needing to read all other chapters.

Brief concluding remarks finally frame this thesis work in a short summarizing section in chapter VII. 


\section{Chapter II. Material and Methods}

\subsection{Chemicals and nematodes}

\subsubsection{Chemicals and reagents}

The chemicals listed below were used in this study and obtained from the indicated sources. Standard chemicals were obtained from either Sigma-Aldrich (Steinheim, Germany), Roth (Karlsruhe, Germany), Merck (Darmstadt, Germany), Boehringer (Ingelheim, Germany), unless otherwise stated. All chemicals were of at least analytical purity.

\subsubsection{Growing conditions}

The strains were grown on nematode growth media (NGM) at $19^{\circ} \mathrm{C}$ and fed with the E. coli OP50 strain if not stated otherwise (Brenner, 1973).

\subsubsection{C. elegans strains and genetic crosses}

Crosses were performed using classical genetic approaches, and the progeny was genotyped by PCR and sequencing. Used and generated reference strains are listed below:

wild type (N2), $d p y-1(e 1), d p y-20(e 1281)$, fat-3(ok1126), tp-12(kaIs12[col-19::GFP]), dpy-1(e1)/tp12 (crossed), rab-2(nu415) [also known as unc-108], rab-3(js49), rab-6.1(tm2124), rab-6.2(ok-2254), rab7/Min1(ok.511), rab-8(tm2991), rab-10(ok1494), rab-11.2(tm2081), rab-14(tm2095), rab-18(ok.2020), rab-19(ok1845), rab-21/cyn-11(ok1879), rab-27(tm2306) [also known as aex-6], rab-28(ok3424), rab-33/taf-11.3(ok1561), rab-35(tm2058), rab-37.1(tm2089), rab-37.2(tm 2089), rab-39(tm 2466), rab-y1(ok 1356), rab-y3 (ok 1871), glo-1(zu391) [represents rab-32/rab-38].

In the following work strains are only named after their mutated gene if the reference strain was used: if strains carrying other mutations were used the allele is directly indicated. 


\subsection{Biomolecular Methods}

\subsubsection{Cuticular specific antibody staining}

C. elegans animals were prepared for immunofluorescence by freeze-fracture on polylysineslides followed by a methanol/acetone fixation at $-20^{\circ} \mathrm{C}$ (Miller, Shakes and Henry, 1995). Slides were processed with milk as a blocking reagent according to the standard immunofluorescence protocol described by (Miller, Shakes and Henry 1995). The DPY-7 antibody was a gift from Iain L. Johnstone (McMahon et al., 2003). Images were captured with the standard confocal microscopy protocol as indicated below.

\subsection{Microscopy}

\subsubsection{Confocal microscopy}

Young adult worms were immobilized in $50 \mathrm{mM}$ sodium azide. Images were obtained on inverted microscope (SP2; Leica) using a $63 \mathrm{x} \mathrm{NA}=1.32$ oil immersion objective at room temperature. Image stacks were captured and maximum intensity projections was obtained using Leica Confocal software (Leica).

\subsubsection{D-Transmission electron microscopy}

A $100 \mu \mathrm{m}$ deep aluminium platelet (Microscopy Services, Flintbek) was filled with E.coli OP50 suspension. About 20 young adult worms were transferred into the chamber and immediately frozen using a BalTec HPM 10. Freeze substitution was carried out in a Leica EM AFS or AFS2 (Rostaing et al., 2004.). Incubations were at $-90^{\circ} \mathrm{C}$ for $100 \mathrm{~h}$ in $0.1 \%$ tannic acid, $7 \mathrm{~h}$ in $2 \% \mathrm{OsO} 4$, and at $-20^{\circ} \mathrm{C}$ for $16 \mathrm{~h}$ in $2 \% \mathrm{OsO} 4$, followed by embedding in EPON at RT (all solutions w/v in dry acetone) (Rostaing et al., 2006). $50 \mathrm{~nm}$ sections were cut using a Leica UC6 ultramicrotome and transferred on formvar-coated copper slot-grids. The grids were placed for $10 \mathrm{~min}$ on drops of 4\% (w/v) uranyl-acetate in 75\% methanol and then washed in distilled water. After air drying the grids were placed on lead-citrate (Reynolds, 1963) for 2 min in a CO2-free chamber, and rinsed three times in distilled water. Recordings of ultra-thin section were performed with a Zeiss-EM 902A equipped with an in-column Henri-Castaing electron energy loss spectrometer. Images were recorded with a cooled 1024×1024 CCD 
camera (Proscan CCD512/1024; Proscan Electronic Systems) using the iTEM Software package (Soft Imaging Solutions).

\subsubsection{3-Dimensional reconstructions from electron tomography}

\subsubsection{Reconstruction from serial sectioning}

Serial recordings of ultra-thin section were performed with the Zeiss-EM 902A (equipped as mentioned above). 3D-data was assembled with the help of the software packages of Reconstruct, rendering was performed with Reconstruct or Amira.

\subsubsection{Reconstruction from electron tomography}

For tomographic reconstruction 10nm colloidal gold particles were applied on both of the sections surfaces (Hoppert, 2003). Single axis tomography recordings were performed with the Zeiss-EM 902A (equipped as mentioned above). Dual axis tomography recording were performed with a Philips $120 \mathrm{kV}$ BioTwin microscope equipped with a 2048x2048 pixel GATAN CCD camera. For single axis tomograms the imaging angle was incremented in a linear fashion $+60^{\circ}$ to $-60^{\circ}$, for dual axis tomography the specimen was tilted from $+60^{\circ}$ to $-60^{\circ}$ in the non-liniear fashion of Saxton (Saxton, Baumeister, and Hahn, 1984). 3D-data was assembled with the help of the software packages of Etomo or PyHST. Rendering was executed directly in Etomo or with Amira. [Philips BioTwin experiments were supervised by D. Riedel]

\subsection{Morphological Assays}

\subsubsection{General growth assays}

Animals were synchronized by alkaline hypochlorite treatment and hatching overnight in M9 buffer (Sulston and Hodgkin, 1988). Animals were then transferred onto freshly seeded plates, allowed to grow for a set length of time and analyzed for body size at maximum magnification under a Leica MZ16FA microscope equipped with a Leica DFC340FX Camera. Images were 
analyzed using the image analysis software XtraCOunt by measuring the length of splines along the midline of the worm. The software is freely available on request.

\subsubsection{Cuticular integrity assay - Hoechst staining}

Worms were washed off NGM plates with standard M9 buffer and incubated in standard M9 buffer containing $1 \mu \mathrm{g} / \mathrm{ml}$ Hoechst 33258 (Sigma) at room temperature for $20 \mathrm{~min}$, followed by several washes with M9 buffer. Worms were a imaged using the UltraviewVOX spinning disk microscope (PerkinElmer).

\subsubsection{Morphology preservation for electron microscopy}

Worms were frozen within the same day and stained with the same solution within the same batch of the freeze-substitution procedure to ensure the highest possible compatibility between the samples. All tissues were recorded at the same magnification and similar body positions. Length and size measurements were performed digitally with Xtra-COunt.

\subsection{Behavioral Assays}

\subsubsection{Movement assay}

Well fed young adults were analyzed for sinusoidal body movements on 1 day old freshly poured, unseeded NGM plates. Feeding animals were transferred and allowed to settle for 10 seconds. Sinusoidal body movements accountable to locomotion were counted for 3 min for each worm and averaged for each strain during forward and backward locomotion.

\subsubsection{Pharyngeal pumping assay}

Pharyngeal pumping rates of wild-type and rab mutant strains were analyzed by transferring L4 worms onto a freshly seeded plate NGM plates with incubation at $20^{\circ} \mathrm{C}$ overnight. 10 healthy adult worms were analyze and assayed twice for $10 \mathrm{~s}$. Counts were averaged for each worm and strain. Strains were declared as impaired when pumping less than three times standard deviation off the wild-type average. 


\subsubsection{Defecation assay}

Defecation cycle lengths of wild-type and rab mutant strains were analyzed by transferring L4 worms onto a freshly seeded plate NGM plates. Two hours after transfer 10 feeding adult worms of each strain were observed for minimum of $10 \mathrm{~min}$ each. Time in between two subsequent p-boc's was analyzed. Counts were averaged for each strain. P-boc's were noted as successful if an expulsion occurred maximal 10 seconds after p-boc. Efficiency rates were calculated as quotient of successful versus total p-boc's.

\subsection{X-ray imaging}

Samples for $\mathrm{x}$-ray imaging were prepared according to previously described electron microscopy standard protocols. X-ray experiments were performed at the European Synchotron Radiation Facility (ESRF) in Grenoble, France and at the Swiss-Light-Source (SLS) in Villigen, Switzerland.

\subsubsection{Experimental Setup - ID21 undulator station at the ESRF}

X-ray fluorescence microscopy of $500 \mathrm{~nm}$ thin sections was acquired by x-ray pointscanning with a Kirkpatrick-Baez (KB) focusing mirror pair, producing a focal spot-size of 330x330 nm at 7,2 keV. An energy resolution of $170 \mathrm{eV}$ was achieved using a energy dispersive detector (Roentec). Energies were recorded up to the energy of the iron k-alpha. Images were scanned with a dwell time of $150 \mathrm{~ms}$ with a step-size of $300 \mathrm{~nm}$. The samples were stained with osmium during the embedding procedures but no counterstain was applied after thin sectioning. Data was processed and visualized with PyMca provided by the beamline instruments software support BLISS at the ESRF. A more detailed description of used the experimental setup can be found in a paper by Tanja Ducic and colleagues (Ducic et al., 2009). A more detailed description of the beamline has been recently presented by Szlachetko and colleagues (Szlachetko et al., 2010.)

\subsubsection{Experimental Setup - ID22NI undulator station at the ESRF}

Holographic imaging was performed at the undulator beamline ID22NI with a $130 \mathrm{x} 140 \mathrm{~nm}$, KB- focused $x$-ray beam at an energy of $17,5 \mathrm{keV}$ (wavelength $=0,708 \AA$ ). The beamline was 
operated in pink mode, i.e. no monochromators were used, with a flux of $10^{11}$ photons per second in the focal spot. Sample position was $25 \mathrm{~mm}$, and detector position was $526 \mathrm{~mm}$ behind the focal plane, resulting a 21-fold magnification. Images were recorded using a FastREadout, LOw-Noise (FRELON) 2000 CCD, equipped with a $24 \mu \mathrm{m}$ thick LSO:Tb scintillator. The optical system was tuned to have a $0.96 \mu \mathrm{m}$ pixel size giving an effective pixel size of 45,6 $\mathrm{nm}$ for our specific imaging setup. A detailed description for the experimental setup can be found elsewhere (Beerlink, 2010).

\subsubsection{Experimental Setup - TOMCAT bending magnet beamline at the SLS}

Tomographic phase contrast imaging was performed with the parallel beam setup at the TOMCAT beamline, at energy output of $17 \mathrm{keV}$ (energetic bandwidth was two percent). Sample position was $54 \mathrm{~mm}$ in front of the detector, a $20 \mu \mathrm{m}$ LuAG:Ce scintillator imaged by a PCO 2000 CCD with including a optical 20-fold magnification objective. 1000 images were captured in a turn of $180^{\circ}$ in a continuous fashion with an acquisition time of $120 \mathrm{~ms}$ per image. Given the $7,4 \mu \mathrm{m}$ pixel size at the detector the effective pixel size was estimated to a final of $370 \mathrm{~nm}$. Reconstruction was calculated from flat- and dark-corrected originals using the modified-bronnikov-algorithmus (MBA) implemented at the beamline server (Groso et al., 2006; Stampanioni et al., 2010). Rendering was performed with the 3D modeling software package of AMIRA.

\subsection{Programming software}

\subsubsection{XtraCOunt}

XtraCOunt single executable file was compiled using the Borland Delphi7 developers environment.

\subsubsection{Rab-Wiki}

The internet-based Rab-Wiki was created with the open source MediaWiki application which was developed by the Wikimedia Foundation. 


\section{Chapter III: Morphological characterization of Rab-GTPases in the nematode $C$. elegans}

\section{Introduction}

To investigate the role of trafficking for each member of the family of small Rab-GTPases in C. elegans, the available null mutant worms had to be first characterized . A series of behavioral as well as functional tests was performed to gain insight into the possible roles and affected pathways of the individual RABs. The results of the tests function to both verify and postulate hypothesis for possible involvements of the individual RABs in trafficking events.

As the nematode genome is mapped and annotated, we first turned to bioinformatic and structural analysis. Having developed a very brought insight into the structural similarities of the Rab-genes and Rab-proteins, we could thus deduce possible redundant pairs of RABs with similar roles. With this description in mind, the worms were morphologically categorized on a light microscopic level to differentiate between severely- and moderately affected rab mutants.

Furthermore the movement (locomotion) of the worms, as well as their feeding and defecation motor programs, were analyzed to find mutants that might have defective muscles or impaired digestive properties, as both of these findings might suggest characteristic trafficking defects. As motor programs are under tight control of neuronal circuits, these functional assay additionally categorize neuronal features. Subsequently, by a gene expression study, the localization of the RABs was determined on fluorescent level with promotor::GFP fusion constructs to pinpoint the RABs to exact tissue involvements.

After combining results from all studies, hypotheses for most of the RAB roles were deduced and a final morphological classification on cellular level using electron microscopy was initiated. A general tissue catalog was generated for purposes of comparing mutants to wildtype tissue-, cellular- and subcellular structures. 


\section{The rab family is highly conserved}

Preliminary mapping of all rabs reveals a rather random distribution of all genes on the six chromosomes (compare Figure 5) but we furthermore wanted to compare their similarity as means for possible redundant roles. For this purpose the structure of the rabs exon/intron composition needed to be clarified. To ease this investigation the graphical tool X-on was developed. It allows display and comparison of the individual rabs. The analysis of sequencing

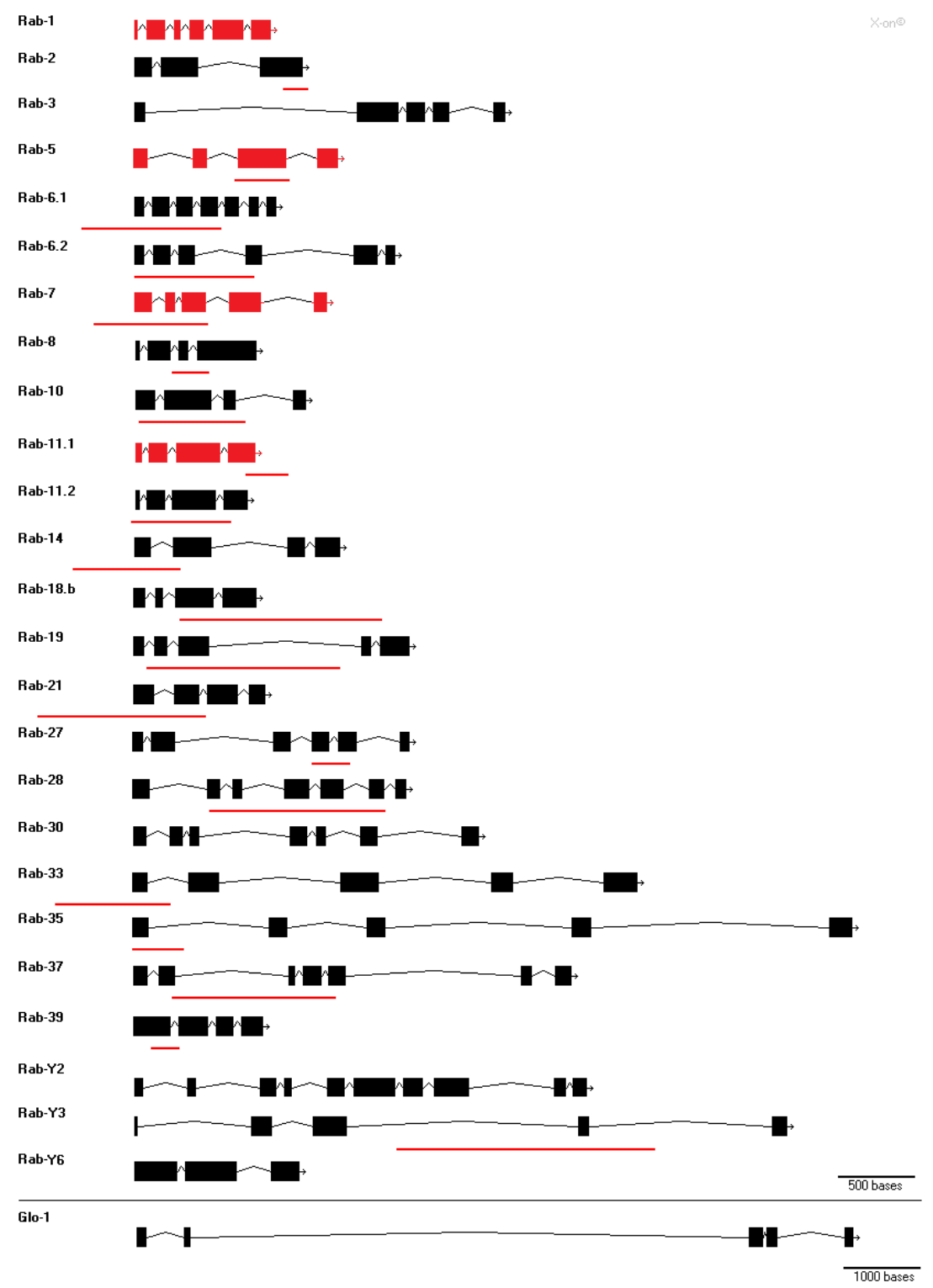

Figure 7: The exon/intron structure of $C$. elegans rabs.

All members of protein class of small proteases consists of a minimum of three but a maximum of 10 exons. The intronic regions cover as low as 10 but also up to 70 percent of the gene. Canonical lethal mutations are shown with red exons and available deletions are underlined red. Only rab-3 and glo-1 carry nonsense mutations. No mutants are available for $r a b-30, r a b-y 2$ and $r a b-y 6$. 
results reveals that the rabs are consisting of minimum of three to a maximum of 10 exons and the intronic regions cover as low as 10 but also up to 70 percent of the gene (Figure 7). As this analysis is purely in-silico, the sequences of all rabs were verified by expression analysis and molecular biological sequencing (Sasidharan, 2011). Twenty eight of the predicted 31 open reading frames showed detectable expression. The available deletion and non-sense mutations mutants, for the further analysis, are listed above.

Comparing the protein sequences alone, a phylogenetic analysis of all Rab-GTPases is possible. A phylogenetic tree displaying the multiple sequence alignment results used to visualize the similarities between the RABs (Figure 8). The multiple sequence alignment calculated by the alignment tool MUSCLE visualizes the result using a distance tree (Edgar, 2004).



Figure 8: Sequence similarity of RABs

Similarity between sequences are shown in a neighbor joining tree as calculated from a multiple sequence alignment. The tree reveals that the isoforms of RAB-6 and RAB-11 are most similar to their partner alleles. The group of RAB-8 and RAB-10, RAB-2 and RAB-14 as well as RAB-3 and RAB-27 show the closest non-allelic identities. Most distant to all other RAB is RAB-28. Similarity is measured as percent identity. Scale bar $=5$ percent identity. 
The analysis of the tree reveals that RAB- 6 and RAB-11 build the most similar clusters with their allele partners. The most non-allelic similarities are shared between the group of RAB-8 and $\mathrm{RAB}-10$ and the group of $\mathrm{RAB}-2$ and $\mathrm{RAB}-14$. A notable similarity is also present for the group of RAB-3 and RAB-27, however this similarity is not as strong as for the previous two pairs. No other RAB seem to share a significant bigger similarity as no other prominent clusters can be detected. Notably, RAB-28 qualifies as the most diverge RAB family member, a feature which interestingly seems to be common to other animal kingdoms possibly indicating the very early divergence of this family member (pers. comm.).

\section{The rab family is analyzed using the model system $C$. elegans}

Having completed a first Rab-characterization on sequence level, we shifted to the morphological classification of the rab mutant nematodes. Before this analysis, a general introduction to the worm, its tissue anatomy and its general body shape is both useful and necessary.

C. elegans is a small, soil nematode that lives in many parts of the world and survives by feeding on microbes, primarily bacteria. As an important model system in biological research, it is used in various fields including genomics, cell biology and neuroscience. The worm provides many research advantages such as short life cycle, compact genome, stereotypical development, ease of propagation and small size (Byerly, Cassada and Russell, 1976).

The adult body plan of the worm is anatomically simple with exactly 959 somatic cells in hermaphrodites and 1031 in males. Due to a very well defined cell linage, their progeny results in completely invariant individuals (Sulston et al., 1983). The nematodes transparency combined with the invariance of cell number and cell position (eutely) thus make the nematode an ideal specimen for microscopy.

The worm's appearance in a standard microscope along with a schematic overview of the tube like anatomy and the important tissues of the worms, is shown below (Figure 9). 

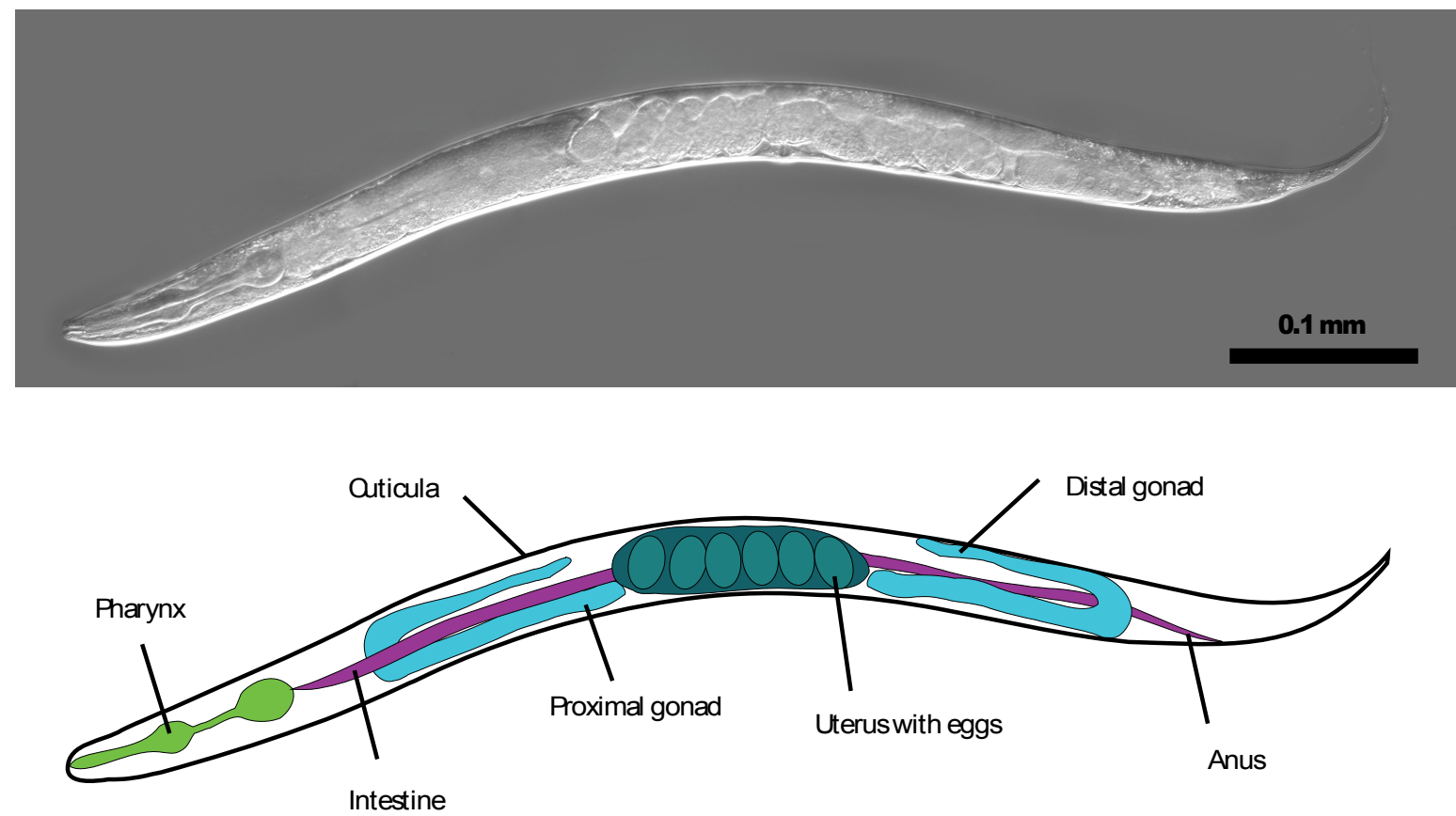

Figure 9: C. elegans body plan

Top: High resolution image assembly of DIC images of a paralyzed, translucent, adult hermaphrodite nematode enabling the identification and characterization of the basic organ structure of the worm. Bottom: Basic schematic anatomical structure of the nematode.

\section{The preliminary analysis of rab mutants suggest individual and common roles of Rab-GTPases}

\section{Two rab mutants are growth defective}

The first obvious morphological feature of the nematodes is their body length. Abnormalities of body length may therefore suggest feeding or digestion defects resulting from a reduction of growth rate $\mathrm{f}$. When comparing the length of the midbody spline of adult hermaphrodite worms (Figure 10), only rab-10 and glo-1 mutant strains display a significant reduction in growth. The size defect of rab-10 is moderate (10\% reduction), but glo-1 mutants are more severe (approximately 20 percent) matching almost the growth results for dumpy-appearing dpy-20 mutants (Hosono et al., 1982). The size investigations furthermore revealed a pronounced growth defect in rab-6.2 mutants but which is penetrant in less than 10 percent of the population and therefore not enough to reduce the averaged values significantly. 


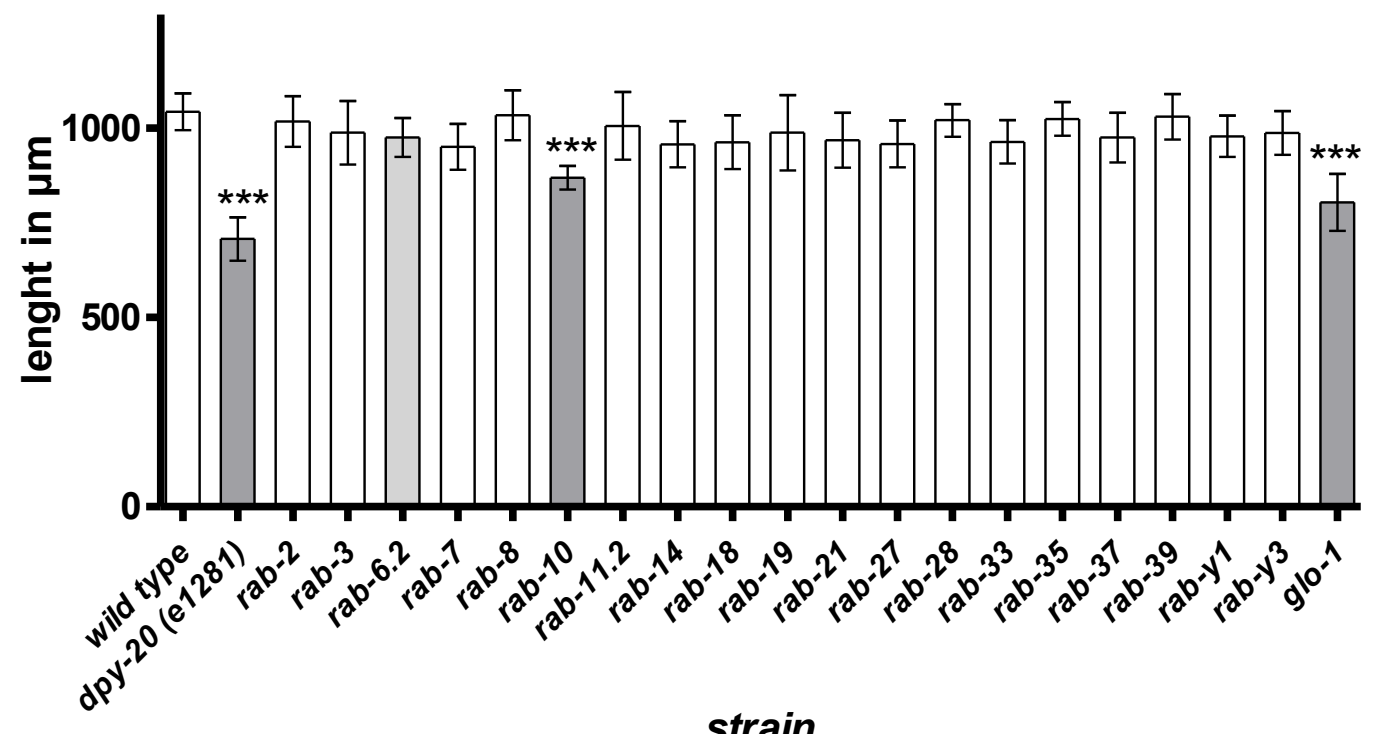

Figure 10: The body length of rab mutants

The average total body length of young adult nematodes was measured. Dpy-20(e1281) was used as a control. All mutant $r a b$-strains except rab-10 and glo-1 mutant animals are found to be like wild type in size. Effected strains are highlighted in dark grey: these rates differ significantly from the average wild type body size $(\mathrm{t}$-test, $\mathrm{p}<0.001)$. Error-bar $=\mathrm{SD} . \mathrm{N}=10$.

\section{rab mutants with pharyngeal pumping defects}

In order check whether the found reduction in growth may correlate with an actual feeding incapability defect, we analyzed the pharyngeal pumping rates of the rab mutant nematodes. The pharynx is the organ that sucks food into the worm, comparable to a vacuum cleaner. In the pharynx, bacterial food is ground up and subsequently pushed into the intestinal lumen. If the pumping of the pharynx is not functioning properly, this might cause a lack of nutrients in the worms and therefore indirectly result in other defects such as the previously found growth limitations. The proper functioning of this motor program requires exact neuronal and muscular control. The pharyngeal nervous system which is sufficient to controls this system is comprised three motor neurons: M3, MC and M4. M3 neurons control the timing of the end of a pump and the initiation of pharyngeal relaxation (Albertson and Thomason, 1976). The MC motor neurons control the excitation rate of the pharyngeal muscle and mediate rapid pharyngeal pumping in response to food (Avery and Horvitz, 1989). The M4 neuron is required for the peristaltic contraction that pushes the food into the direction of the intestine 
(Avery and Horvitz, 1989). M3 neurons function with $\gamma$-aminobutyric acid - GABAergic (Dent et al., 1997) and MC neurons function with acetylcholine - cholinergic (Raizen at al., 1995). M4 are cholinergic neurons but employ other neurotransmitters as they can not be fully blocked by cholinergic agonists (L. Avery, pers. comm. on www.wormbase.org). Assaying the pharyngeal pumping rate might thus reveal neuronally compromised rab mutants.

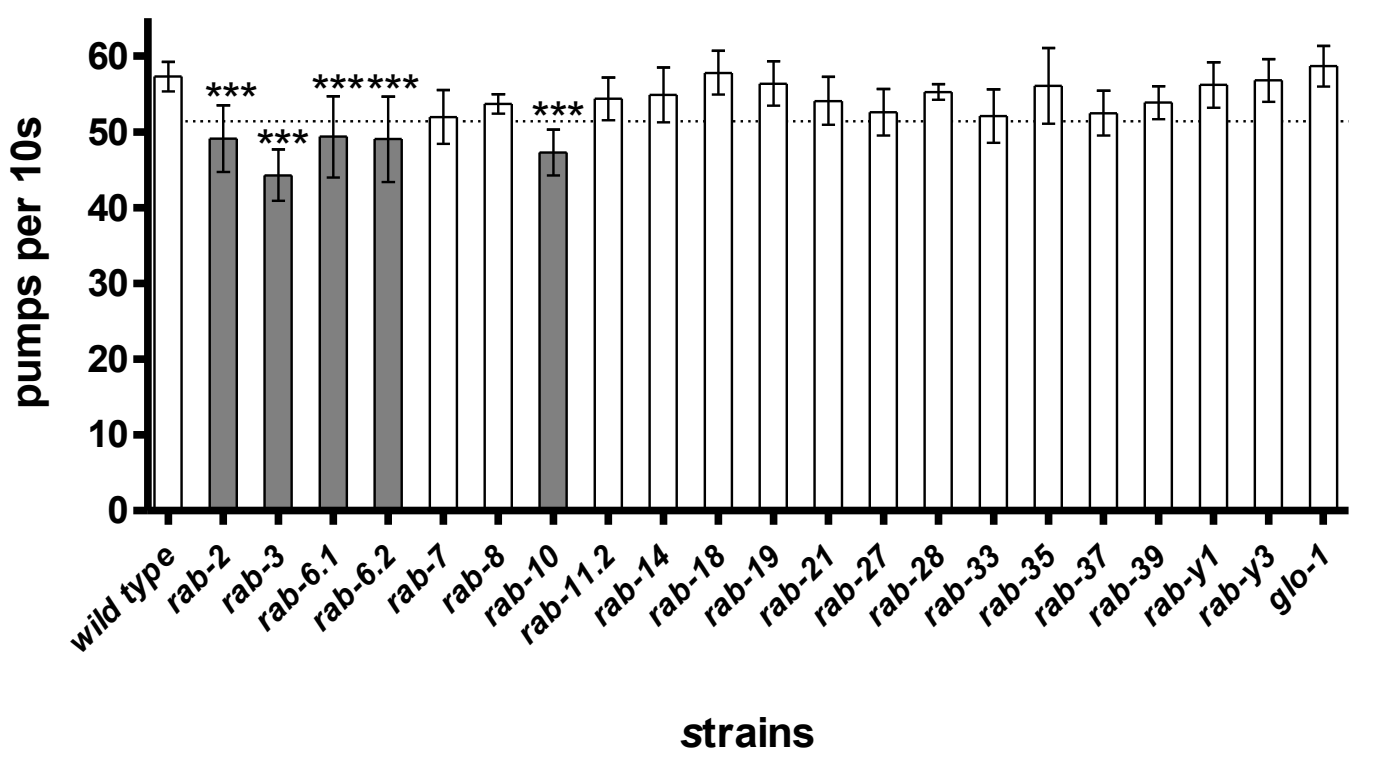

Figure 11: Pharyngeal pumping of feeding of rab mutants

The average pumping rates for feeding adult nematodes was examined and found to be severely affected in rab-3 mutant background. rab-2, both rab-6 and rab-10 are less affected. All other rab mutations seem to have no effect on the pharyngeal pumping rate. Affected strains are highlighted in dark grey, all these rates are significantly different from the wild type rate by more than 3 times standard deviation difference and $\mathrm{p}<0.001$ (t-test). Errorbar $=$ SD. $\mathrm{N}=10$.

When analyzing the pharyngeal pumping rates for well fed, undisturbed worms, a number of rab mutants displayed reduced pumping (Figure 11). A very severe pumping defect could be measured for rab-3, confirming previous investigations (Nonet et al., 1997). The pumping defect here results from the well- characterized malfunctioning of $r a b-3$ as a tethering factor for synaptic vesicles in synaptic transmission. In addition to rab-3, the mutant of rab-2, rab-6.1 and rab-6.2 as well as rab-10 showed a decreased pumping rate. Of these, only the rab-2 mutant has been previously connected to this function (Sumakovic et al., 2009). Interestingly, the sizeaffected rab-10 was among the group of affected mutant, while glo-1, previously marked with a size defect, was not. 


\section{The defecation motor programs are defective in a number of rab mutants}

While pharyngeal pumping regulates food intake, a second motor program regulates defecation. Underlying the defecation motor program is an oscillator which also functions in the intestinal cells of the animal (Teramoto, and Iwasak, 2006).

The rhythmic defecation cycle periodically repeats three consecutive steps roughly every 50 seconds. First, the contraction of the posterior body wall muscles (pBoc), which compresses the gut contents into the anterior part of the intestine. Second, the contraction of the anterior body wall muscles ( $\mathrm{aBoc}$ ), which pushes the gut contents to the posterior part of the intestine. The final step is the contraction of the enteric muscles and relaxation of the enteric sphincter, which results in the expulsion (Exp) of feces. AVL and DVB, both GABAergic neurons, together regulate this enteric muscle contractions (Liu and Thomas, 1994). This cyclic appearing mechanism involves periodic calcium release and subsequent intercellular calcium waves, which in turn regulates the muscle contractions that create the defecation action (Peters et al., 2007, Kwan et al., 2008). As many control and execution mechanisms have to be functioning properly for the accurate regulation of this defecation cycle a screening with the rab mutants has been initiated.

The assay was separated into two distinct investigation. In a first step, the cycle length (inter pBoc interval) was examined and in a second step, the expulsion efficiency was monitored (Figure 12). 


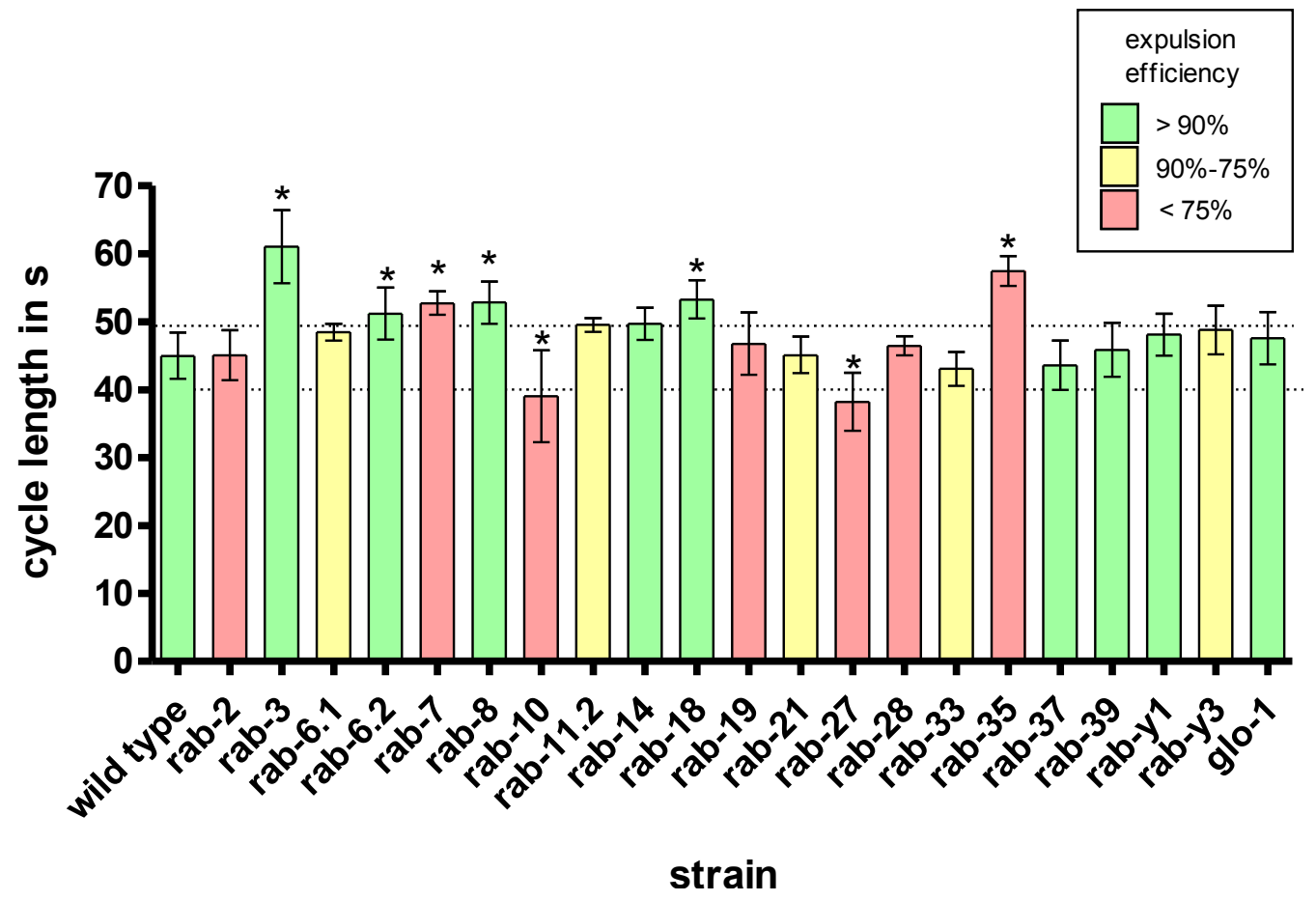

Figure 12: Defecation cycle length and expulsion efficiency of rab mutants

The average defecation cycle lengths for feeding adult nematodes was examined and found to be raised rab-3, rab6.2, rab-7 rab-8, rab-18 and rab-35 mutant background. rab-10 and rab-27 mutants exhibit faster rates. All other rab mutations display no drastic effects on the motor program of the defecation cycle. Timing from two subsequent pboc's was examined. Strains were declared as effected if the averaged cycle lengths is altered by $5 \mathrm{~s}$ or more. An asterisk marks all rates significantly different from the wild type (t-test, $\mathrm{p}<0.005)$. Expulsion efficiency is indicated by colors. Green indicates no defect, yellow a mild defect and red indicates a severe defect. Experimental study has been aided by N. Sasidharan and M.Sumakovic. $\mathrm{N} \geq 100$. Error-bar $=$ SD.

For cycle length, we measured prolonged intervals for rab-3, rab-6.2, rab-7, rab-8, rab-18 and rab35, where rab-3 and rab-35 are the most delayed. rab-10 and rab-27 showed an abnormally fast defecation cycle. Here again the rab-3 mutant suggested that impaired synaptic transmission may cause alterations to the motor programs. In addition to the cycle length, the expulsion efficiency of the nematodes was found to be severely reduced (smaller $75 \%$ - indicated in red) for rab-2, rab-7, rab-10, rab-19, rab-27, rab-28, rab-35.

For rab-27, also know as aex-6, the cycle length reduction in these mutants may result from an improper resetting of the calcium concentrations (Thomas et al., 1990).

Three other phenotypes that were remarkable were rab-3, which showed a radically slowed expulsion cycle with normal cycle lenghts and the two expulsion defect mutants rab-10 and rab35 which have a drastically faster or slower cycle length respectively. As mentioned previously, 
rab-3 has a well studied synaptic tethering defect. which explains the delay in execution of the motor program. However, the role of rab-10 and rab-35 in defecation regulation have not been investigated so far. Noteworthy is that rab-10 phenocopies rab-27 in its behavior, even if not as drastically, which in turn might give an explanation for this defect. Even more remarkable is that rab-35 shows a expulsion defect but has, counter-intuitively, a prolonged defecation cycle. The mechanistic explanation for this phenotype has yet to be determined.

\section{Only rab-2 shows drastic locomotory impairment}

As the most obvious worm behavior, movement capability was analyzed in the rab mutant worms. The nematodes move in a sinosoidal fashion. This coordinated movement requires accurate neuronal coupling and muscle function. 6 major classes of motor neurons, which are responsible for locomotion: VA, VB, VD, DA, DB and DD (Von Stetina et al., 2006). All Cell bodies of these neurons are located in the ventral nerve cord (VNC). The sinusoidal movement of the worm is mediated by the simultaneous contraction and relaxation of the ventral and dorsal muscles. VA, VB, and VD form synapses with ventral muscles, whereas DA, DB and DD send contralateral commissures to form synapses at the dorsal side (Riddle et al., 1997). The VA, VB, DA and DB motor neurons are cholinergic, whereas the VD and DD motor neurons are GABAergic. With this set of neurons an activation of one muscle side and contralateral inhibition of the other side can be achieved (Von Stetina et al., 2006).

Through testing of the locomotion efficiency of the worms, we found that only rab-2 mutants displays drastic reduction (50 percent). rab-2, originally described as unc-108 (uncoordinated), has been known to display this defect. However it was only recently that we could attribute this defect to a neuronal dense core vesicle defect (Sumakovic et al., 2009). As depicted below no other mutant displayed a quantifiable defect in locomotion (Figure 13). 


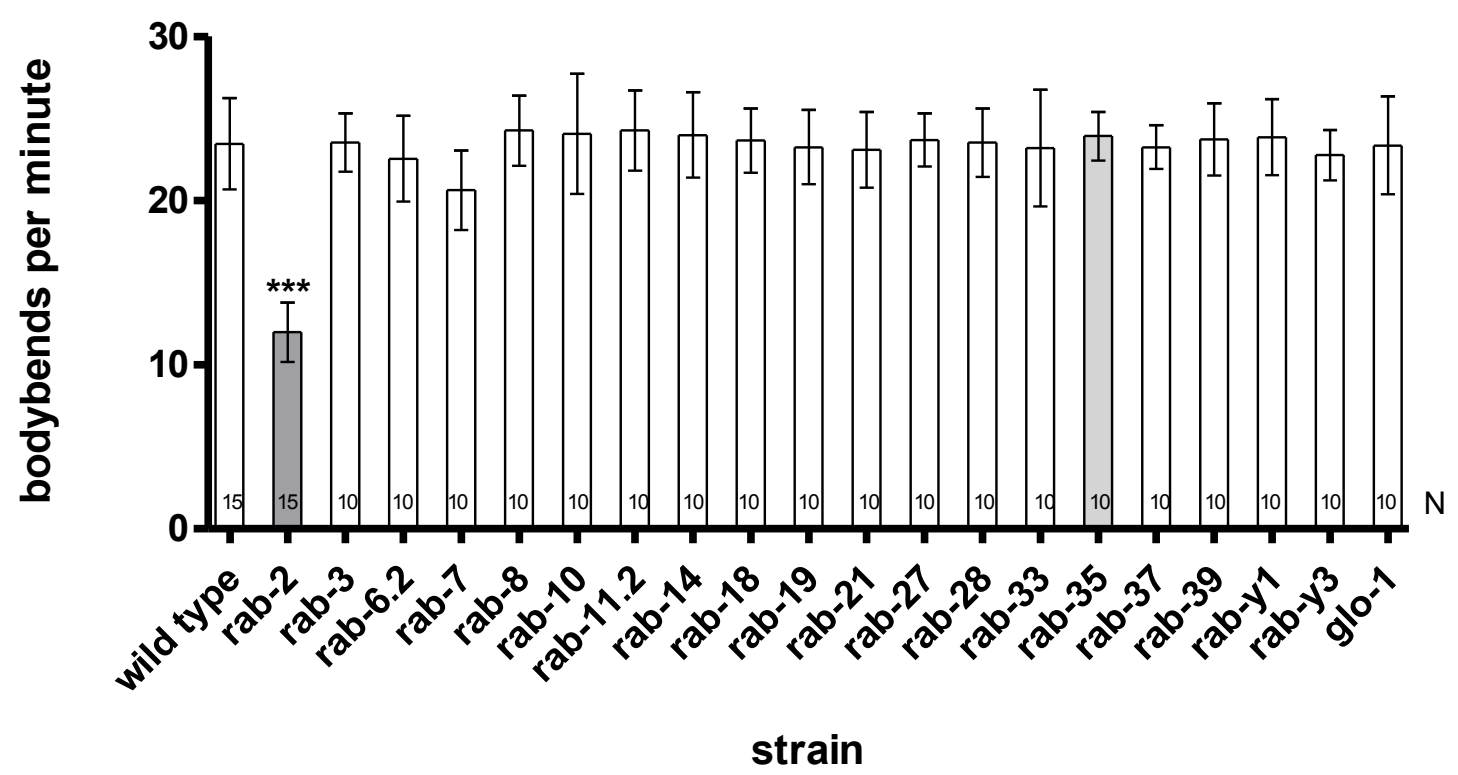

Figure 13: Movement analysis of rab mutants

Other than the already characterized rab-2 mutants (unc-108) no other rab mutant shows a significant (t-test, $\mathrm{p}<0.001$ ) decrease in body movements (bodybends). Note that despite its normal rate of bodybends the rab-35 mutant is impaired in proper forward locomotion. This mutant moves normally on seeded plates but is appearing to swim in position position when transferred to 1 day old unseeded plates. Error bars indicate standard deviation. Significance is marked with $* * *$ for $\mathrm{p}<0.001$. Experimental study has been aided by N. Sasidharan and M.Sumakovic. Error-bar = SD.

Interestingly, rab-35 mutant worms display a reduced capability to propel themselves on unseeded plates. Their entire body appears to swim over the ground and only the additional friction given by seeded E. coli allows them to properly crawl. Since the movement on seeded plates therefore appears no different from wild type this might hint to structural aberrations in the cuticle of these rab-35 mutant worms.

\section{Cuticle integrity is not maintained in rab-6-mutants}

To test the structural integrity of the cuticle of the rab mutants a slow-diffusing, intercalating, fluorescent dye was used. Mutants were bathed in the dye and imaged shortly thereafter. The presence of stained nuclei in the body wall muscles would therefore indicate that the natural, barrier function of the cuticle is impaired. The results of this assay are given below (Table 1). For deeper understanding of cuticle architecture the readers may refer to chapter $\mathrm{V}$, where a mutant carrying a cuticular defect is analyzed in more detail. 


\begin{tabular}{|c|c|c|}
\hline$r a b$ & mutant allele & Hoechst staining \\
\hline wild type & $\mathrm{N} 2$ ( - control) & - \\
\hline bli-3 & e767 (++ control) & ++ \\
\hline$r a b-2$ & nu415 & - \\
\hline$r a b-3$ & js49 & - \\
\hline$r a b-6.1$ & $\operatorname{tm} 2124$ & ++ \\
\hline$r a b-6.2$ & ok 2254 & ++ \\
\hline$r a b-8$ & $\operatorname{tm} 2991$ & - \\
\hline$r a b-10$ & ok 1494 & - \\
\hline$r a b-11.2$ & $\operatorname{tm} 2081$ & - \\
\hline$r a b-14$ & $\operatorname{tm} 2095$ & - \\
\hline$r a b-18$ & ok 2020 & - \\
\hline$r a b-19$ & ok 1845 & - \\
\hline$r a b-21$ & ok 1879 (\& cyn-11) & - \\
\hline$r a b-27$ & $\operatorname{tm} 2306$ & - \\
\hline$r a b-28$ & ok 3424 & - \\
\hline$r a b-33$ & ok 1561 (\& taf-11.3) & - \\
\hline$r a b-35$ & $\operatorname{tm} 2058$ & $+?$ \\
\hline$r a b-37$ & $\operatorname{tm} 2089$ & - \\
\hline$r a b-39$ & $\operatorname{tm} 2466$ & - \\
\hline$r a b-Y 1$ & ok 1356 & - \\
\hline$r a b-Y 3$ & ok 1871 & - \\
\hline glo-1 (32/38) & zu 391 & - \\
\hline
\end{tabular}

Table 1: Staining results after 20 min Hoechst 33258 bathing

Most mutant rab strains maintained the capability to exclude the dye, however rab-6.1, rab-6.2 were showing nuclear staining. Very evident in their late adulthood, most of these worms rupture rather than die of aging. A staining with antibodies that marks circumferential, body constricting fibers in these mutant strains lead to the preliminary and not yet verified hypothesis, that their circumferential fibers may not be properly connected. This imperfect radial constraint is likely the cause of the observed increased busrting rate during adulthood. A tendency of rab-35 mutants to acquire a weak stain has been noticed not comparably strong as the positive controls. Further tests in this area have not yet led to conclusive results. 


\section{Expression of Rab-GTPases is tissue specific}

To clarify the tissue specific roles of all $r a b$ promotor-GFP-fusion constructs were generated to visualize the tissue-specific expression pattern with fluorescent microscopy. The expression analysis reveals that most of the rabs are ubiquitously expressed, while some rabs are expressed only in certain tissues (Figure 14), such as among others the purely neuronal RAB-3 or the intestinal RAB-21.
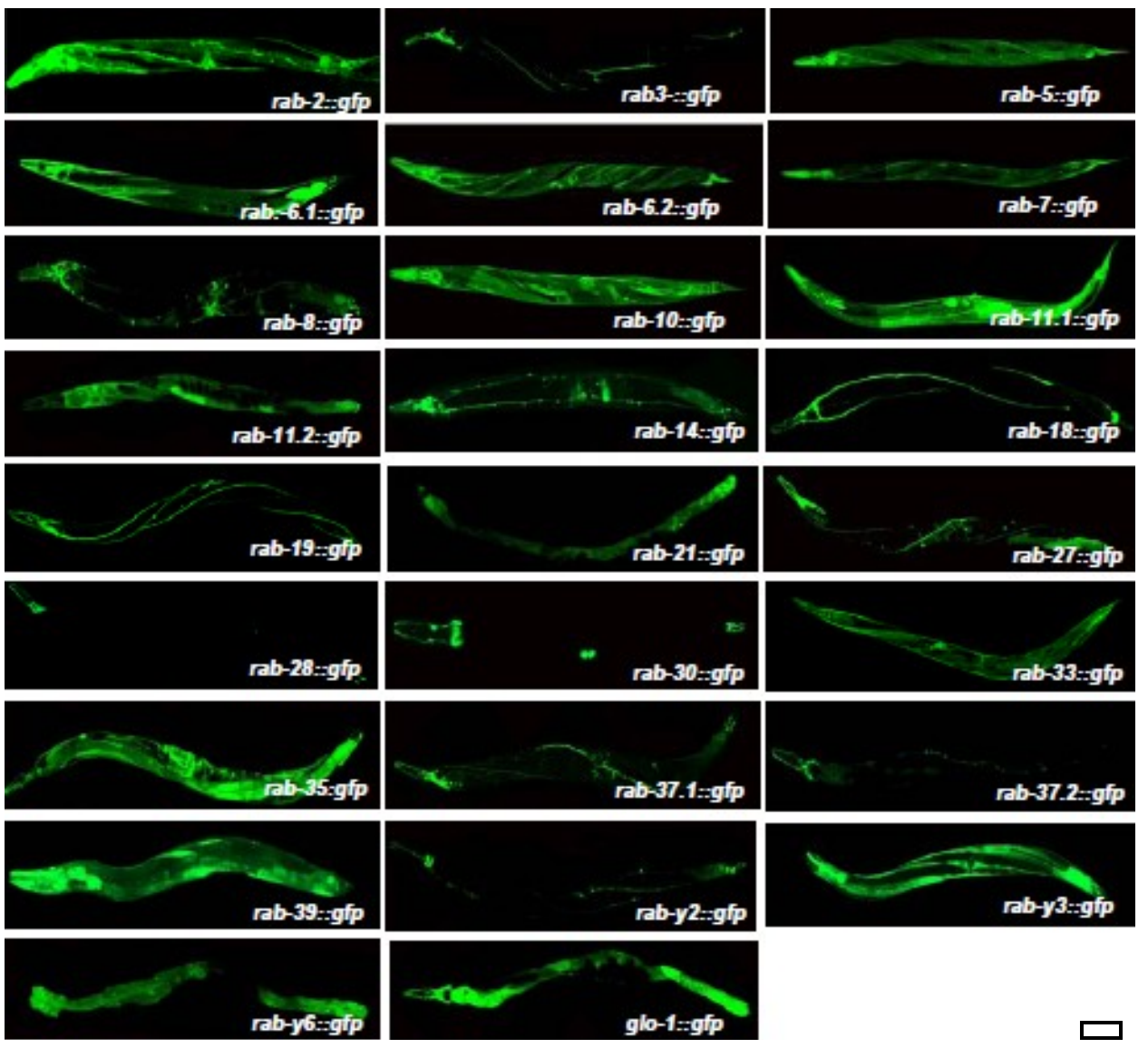

glo-1::gfp

Figure 14: Expression patterns of $C$. elegans rabs

Rab-GFP promotor fusions in combination with fluorescent microscopy allow the detection of the expression pattern of rabs in C. elegans. Some rabs are tissue specific and the majority of rabs are expressed preferentially in neuronal and intestinal tissues. Scale $=100 \mu \mathrm{m}$. [ Experiment was conducted by Nikhil Sasidharan].

With the help of this expression atlas a morphological analysis on subcellular level can now be initiated. The investigation is carried out with electron microscopy together with the most advanced, tissue preserving, embedding methods. The results of these experiments will be presented in the next section. 


\section{High Pressure Freezing preserves $C$. elegans tissues to an in vivo like state}

\section{Anatomy can be accurately revealed by electron microscopy}

The anatomical description of the nematode has been completed at the electron microscopy level, as for example the entire neuronal network and mapping has been categorized by this technique (White et al., 1986). The recent advances in this field have resulted in techniques which are called high pressure freezing and slow freeze-substitution. These techniques allow a in-vivo like preservation of the nematode tissues for imaging with electron microscopy (Rostaing et al., 2004). Having established this technique in our laboratory we have used it to investigate the native appearance of the tissues in C. elegans (Figure 15).

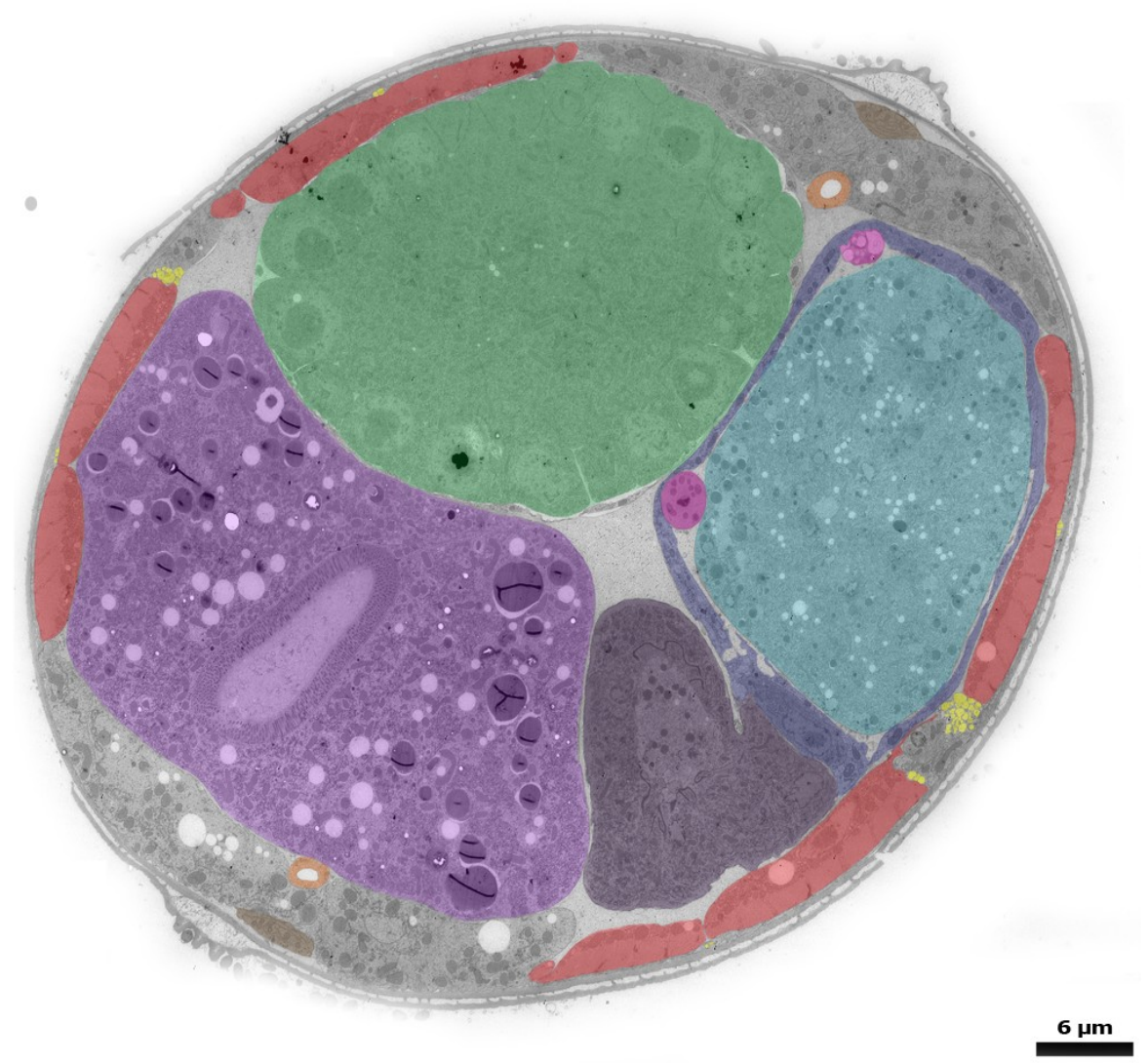

Figure 15: Cross sections through the midbody of the nematode

High pressure frozen and freeze-substituted C. elegans samples are preserved in an in-vivo like state. Internal organs can be clearly differentiated on thin section in the TEM. Red -Muscles; Yellow - Nerves; Green - Early gonad; Cyan - Late gonad; Blue -Uterine sheet cell; Dark purple - Spermatheca; Pink - Sperm cells; Purpe Intestine; Orange - Secretory $(\mathrm{H})$ cell; Brown - Seam cell; Gray - Hypodermis; Light gray - Pseudoceolomic (body) cavity. 
The cross-sections through the nematode (as depicted in figure 15) give insight into several distinct tissue of specific function. The preliminary steps of our analysis deal with a high resolution investigation of the major tissues in $C$. elegans. With the generated images a tissues atlas has been created to function as a reference for the native architecture and morphology (Figure 16). The invariant development and constant cell number and completely similar body morphology of each of the nematodes eases the detections of alterations that are due to gene mutations.
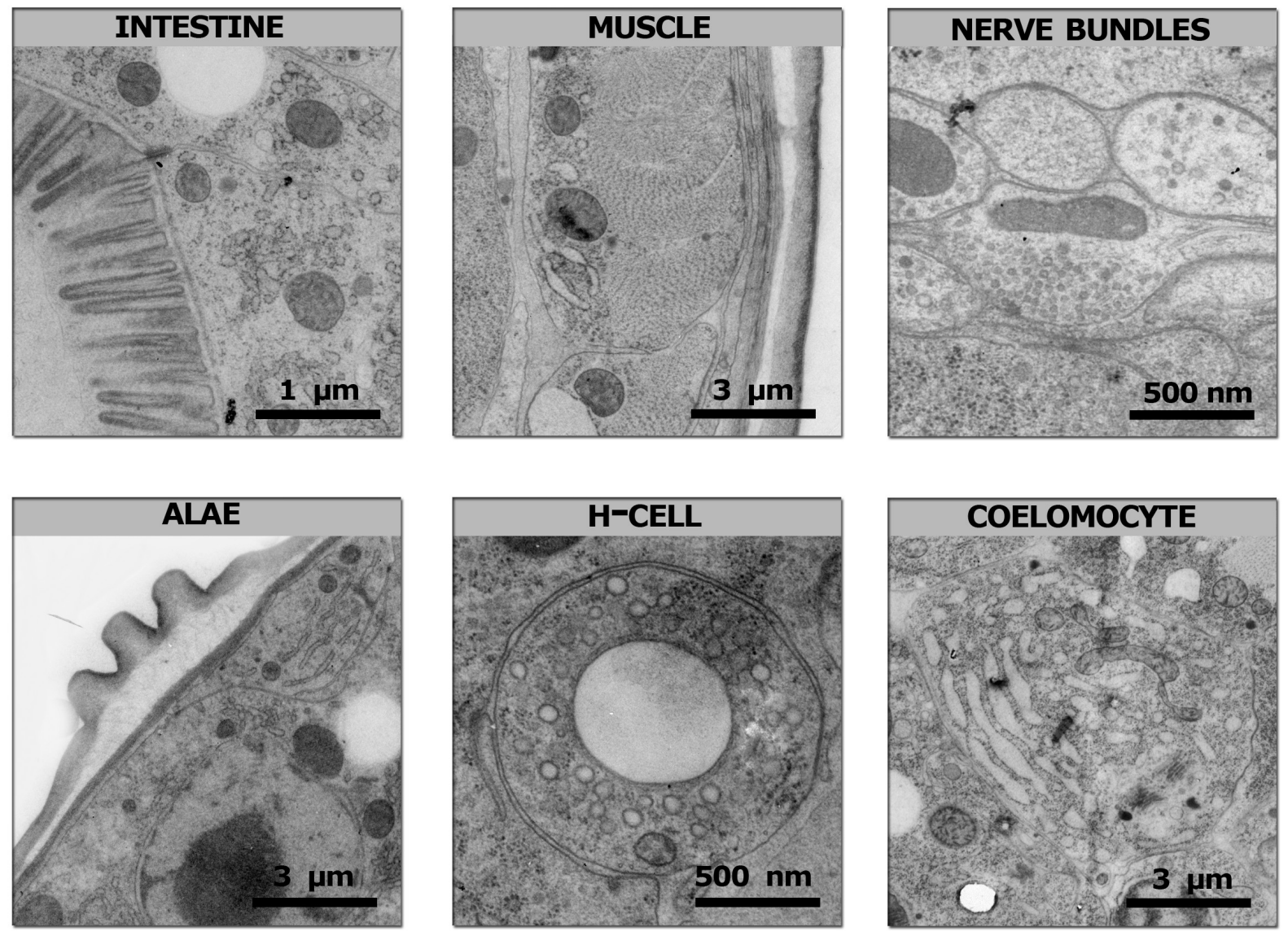

Figure 16: High resolution TEM tissue catalog

TEM images of high pressure frozen nematodes show the native, in-vivo like architecture of internal organs in the nematode. These images are used to create a catalog for the nematode tissues - tissue type is indicated on top. All pictures are cross-sections through the worm. Nerve bundles are imaged from longitudinal slices. 


\section{Tissue reconstructions can be achieved with the help of serial sectioning}

The sectioning of the embedded samples only allows a two dimensional (2D) view into the tissues. However, the reconstruction from multiple sections from a worm in a serial fashion allow for three dimensional (3D) tissue analysis. With the help of this technique entire organ structures can be revealed which would have been hidden in a pure $2 \mathrm{D}$ visualization.

As an example, reconstructions from serial section of a ceolomocytes (Figure 17) and a synaptic nerve terminal (Figure 18) are presented below to illustrate the advantages of a 3D tissue reconstruction.

Coelomocytes, are continuously working scavenger cells that non-specifically endocytose fluid from the body cavity (pseudocoelom) of the worm. Single sections of a coelomocyte show multiple vesicular elements of various shape but only the $3 \mathrm{D}$ reconstruction is able to reveal an interconnected network of tubular nature (Figure 17).
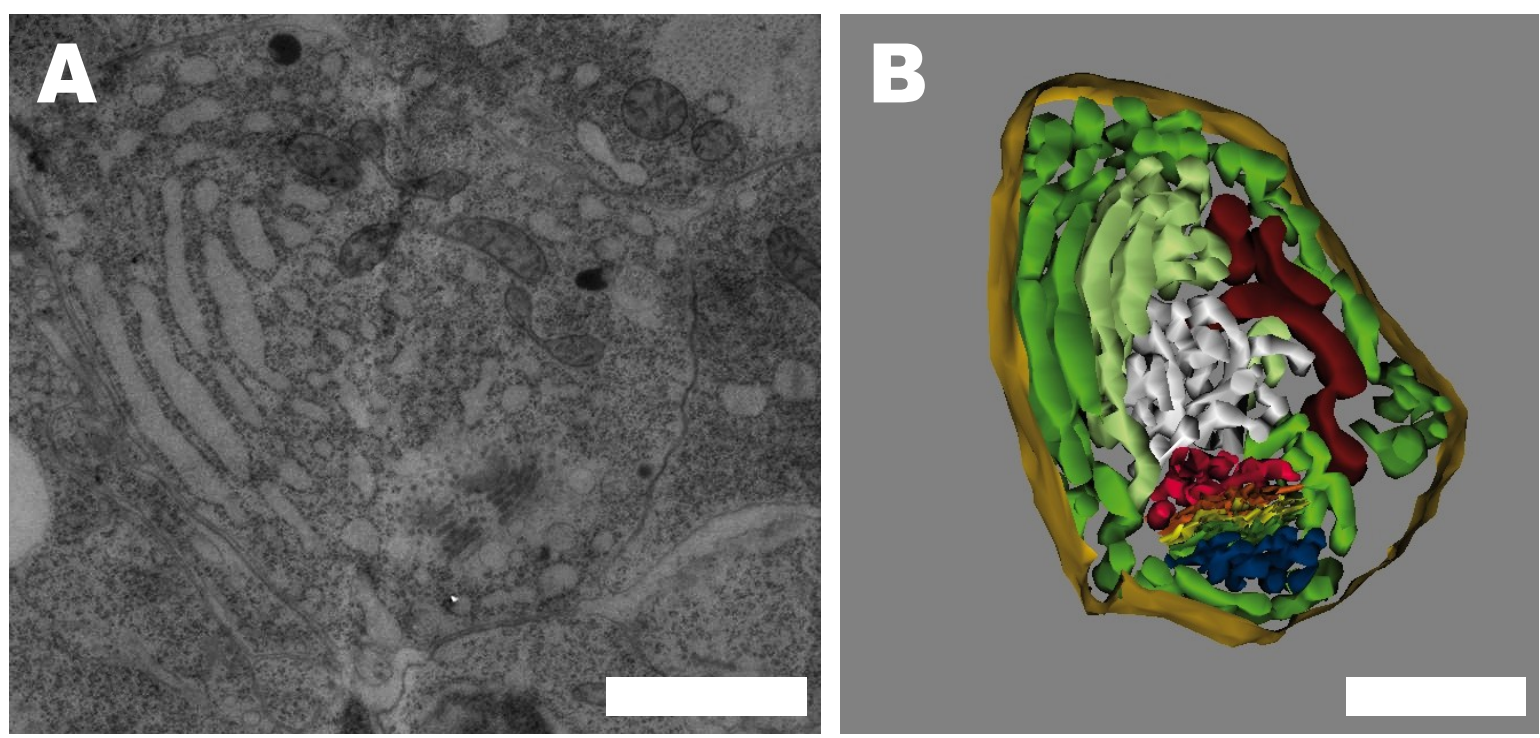

Figure 17: Serial section and $3 D$ representation of a coelomocyte

TEM images of serial sectioned coelomocytes (A) and other tissues allow the reconstructions of the 3D volumes of entire tissues or tissue sections (B). Scale bar $=3 \mu \mathrm{m}$. 
In reconstructions of nematode nerves using serial sectioning, the vesicle distributions within synaptic terminals (Figure 18) can be visualized.
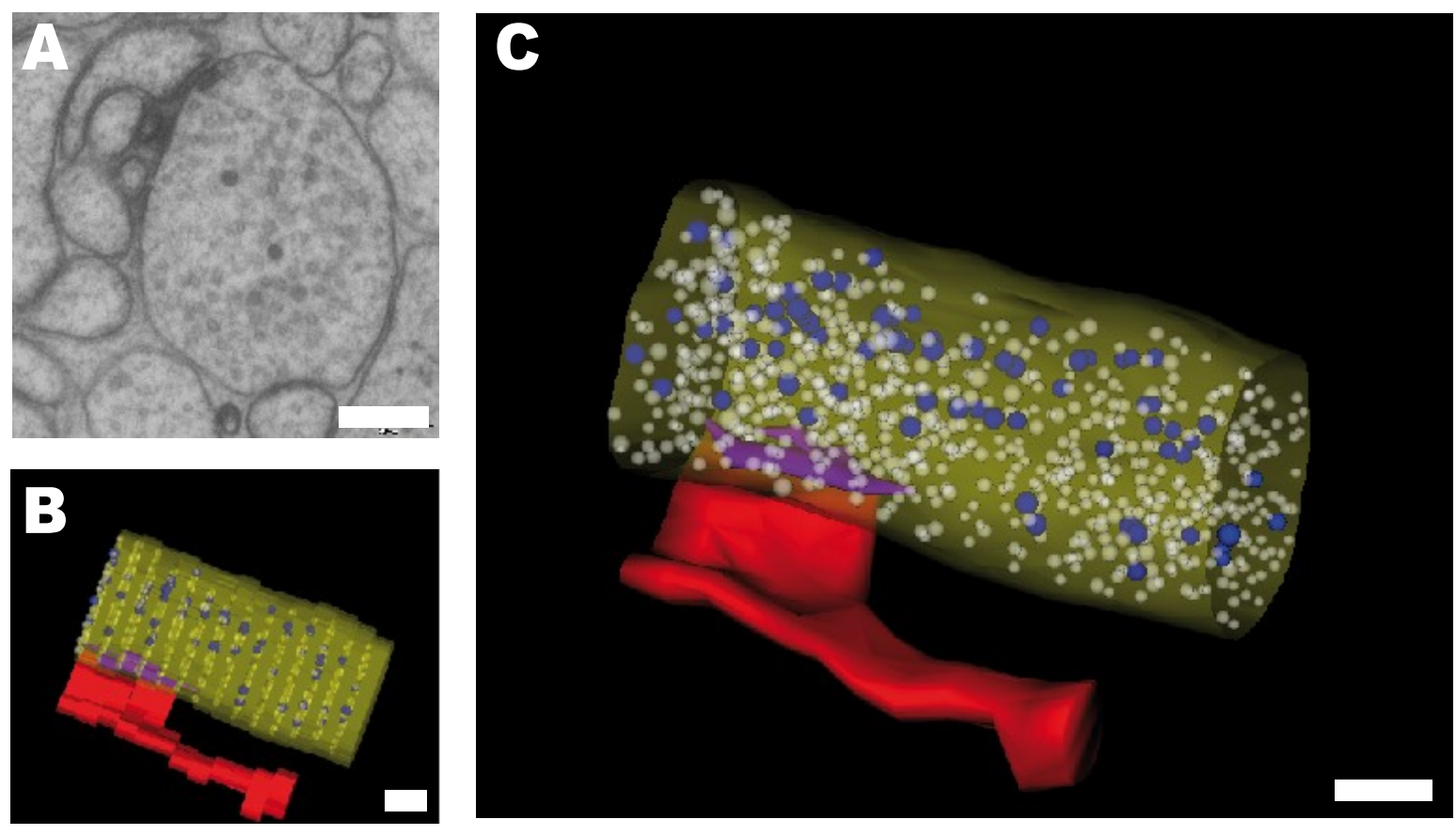

Figure 18: Serial section and 3D representations of a synaptic nerve terminal

(A) TEM images of serial sectioned nerve terminal. (B) The true slice representation illustrates the low z-axis resolution of the serial section approach. (C) Visually more appealing but not more accurate results can be achieved by data smoothing. Indicated tissues: red $=$ muscle arm, yellow $=$ nerve, purple $=$ synaptic density, white $=$ synaptic vesicles, blue $=$ dense core vesicles. Scale bar $=200 \mathrm{~nm}$.

For tissues with subcellular structures of sizes far greater ( $>100$ times) than the sectioning thickness, such as the tubular structures in coelomocytes, the reconstruction technique from serial sections enables the understanding of the $3 \mathrm{D}$ architecture of the tissue and is therefor superior to the plain 2D consideration. However, the limits of this reconstruction techniques are reached if the reconstructed tissues, such as in case of the nerve terminal, are of similar sizes ( $\leq 10$ times) to the section thickness (as indicated in Figure 18B).

However, the high-pressure frozen samples are of most interest at high-resolutions, where the structure of subcellular organelles can be investigated. In the next section we thus focus on the characterization of subscellular features in 2D and 3D using the example Golgi apparatus. 


\section{The Golgi machinery has tissue specific adaptations}

The subcellular anatomy can be analyzed with electron microscopy

In subcellular localization studies we have found multiple partial and full overlaps of RAB presence with Golgi marker. The Golgi represents the central trafficking organelle and is thus of great interest for trafficking studies in general but especially for our rab studies. During the analysis of various tissues, the Golgi apparatus was found to show distinct adaptation to the tissues which most likely hint to the overall functional activity of the apparatus. The architecture of these tissue specific Golgis is shown below (Figure 19).
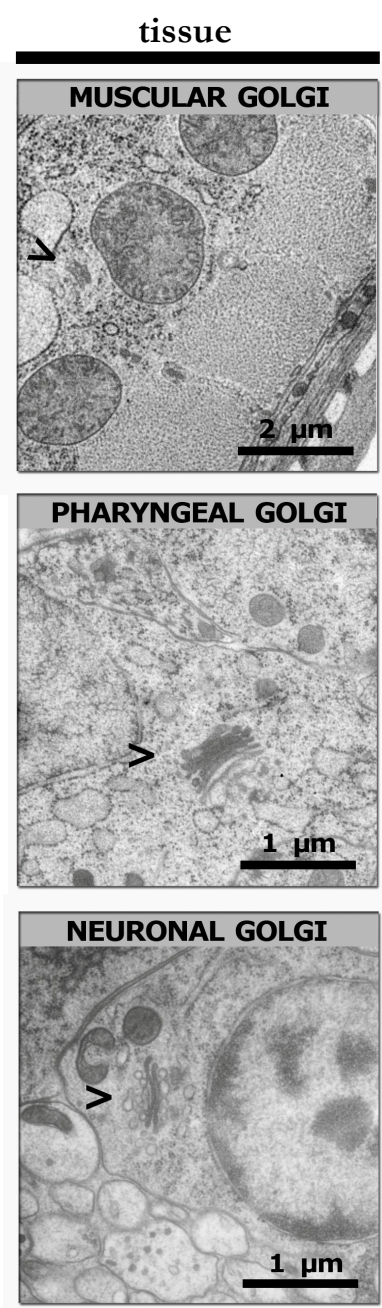
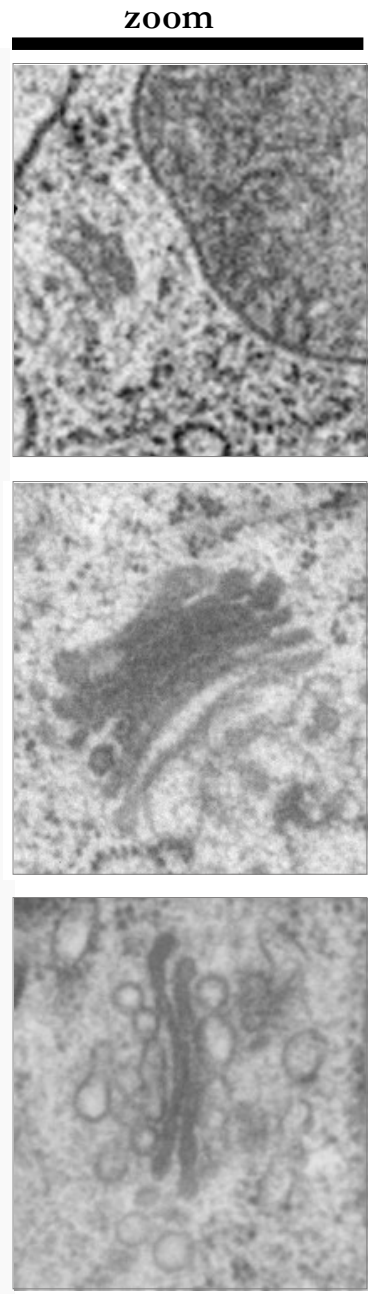
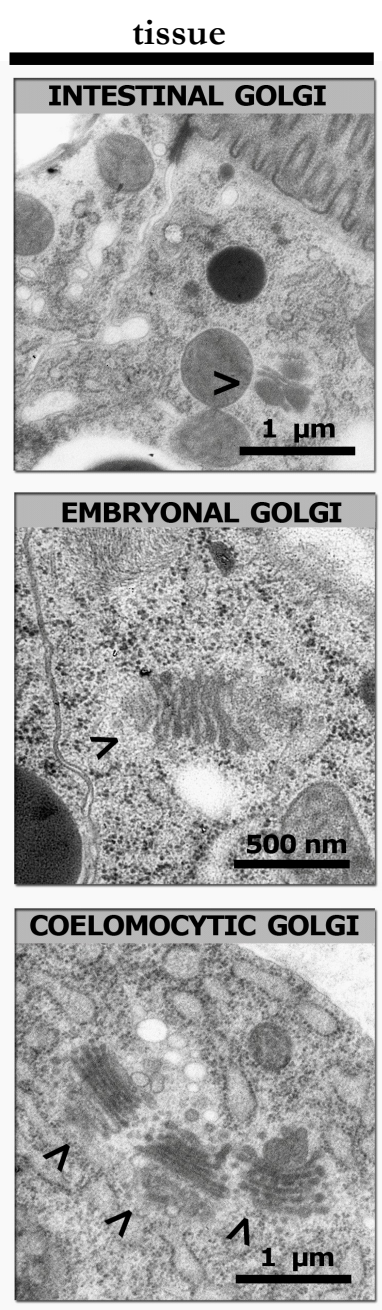
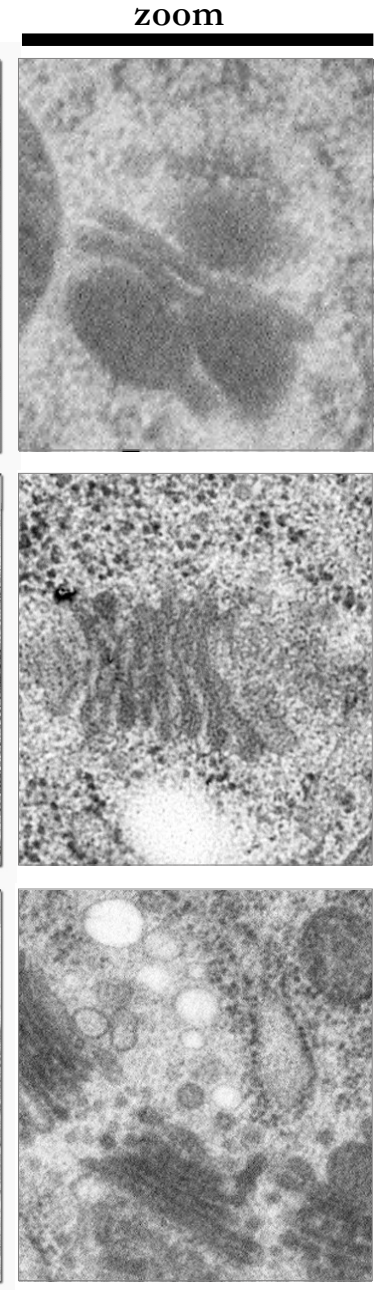

Figure 19: Shape and size of the Golgi in different tissues

TEM images of ultrathin sections with subcellular resolution reveal that the form of a Golgi is strongly dependent on its cell/tissue type. The intestinal Golgis consist of a 3-5 cisternae which show swollen ends. The muscular Golgi is consists of 3 vesicular cisternae but the pharyngeal Golgi shows 4-6 cisternae despite of its muscular tissue nature. The neuronal Golgis appear with 3-4 stacked cisternae text book alike while embryonal, seam cell, endothelial and coelomocytic Golgis have multiple cisternae. 


\section{The subcellular anatomy can be analyzed with electron microscopy}

As depicted in the figure above, the very abundant intestinal Golgis are consisting of a three to five cisternae that often show swollen ends. The rare muscular Golgi is consisting of three cisternae that appear like three big vesicles on a string. The typical two neuronal Golgis appear with three to four medial cisternae associated with a clear ERGIC and TGN. Embryonal, seam cell, endothelial and coelomocytic Golgis have multiple cisternae (often around 10 and up to 15) and the ceolomocytic Golgis are thereby often associated in groups of two, three or four individual Golgis. The pharyngeal Golgi despite of its muscular origin has four to six cisternae and is flattened in contrast to the vesicular architecture of muscular Golgi from the body-wall.

\section{Electron tomography allows subcellular reconstructions}

A 3D reconstruction of Golgis from serial sections is not feasible. The generation of ultrathin slices is working accurate down to about $30 \mathrm{~nm}$, a Z-resolution which is not feasible to reveal the entire anatomy of the pleomorphic Golgis. To access these structures a direct approach towards three-dimensionality is necessary. The technique of electron tomography enables a high resolution 3D view of small sized tissue samples. Despite of the fact that semi-thick sections of $250 \mathrm{~nm}$, a Golgi cannot be seen as a total but the resolution of the generated tomograms exceeds that of a serial-slice attempt by far. A reconstruction of Golgi with a zresolution in the nanometer range is possible (Figure 20).

In a first attempt wild type tissues were categorized. Having established these techniques of preserving, embedding and imaging an investigate all mutants of the rab family has been initiated - a procedure which is however still not completed. One third of all mutants have been imaged so far and the remaining mutants are currently processed. If the Golgi-located RABs alter the shape of the central trafficking organelle the results of this study will lead to the better understanding of how RABs integrate into the trafficking machinery. Similar studies with other organelles and their associated RABs are also conceivable. 

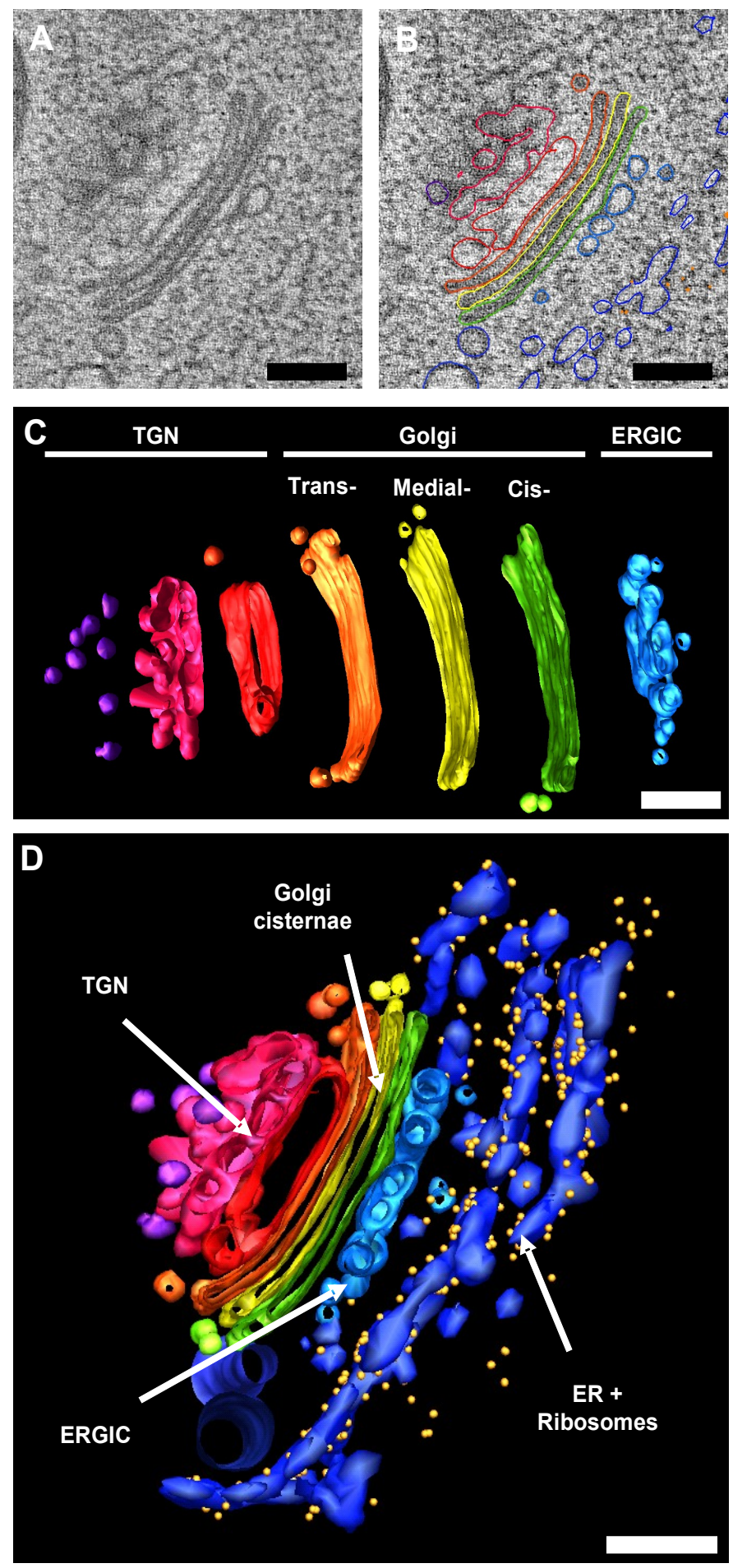

Figure 20: Electron tomograms and3D model of a Golgi

(A)Virtual slice and (B)model contours of a tomographic reconstruction from tomogram of a $250 \mathrm{~nm}$ thin EPON section of an intestinal Golgi. (C) Rendered 3D model of all Golgi stacks. (D) Colored full 3D model of the thin-section. Blue - ER; Gold- Ribosomes; Cyan - ERGIC; Green - Cis cisternae; Yellow - Medial cisternae; Orange - Trans cisternae; Red,Pink,Purple - TGN with dissociating vesicles. Scale bar $=120 \mathrm{~nm}$. The tomogram was recorded with Dietmar Riedel and a Philips $120 \mathrm{kV}$ BioTwin microscope at the MPI-BPC in Göttingen. 


\section{A open source Rab-Wiki comprises data from the Rab-Atlas study}

To readily display and access the data, a internet-based Wiki-platform was developed (www.enig.de/wiki_seimer). Here all primary data is displayed and can be compared between the individual mutants. As one representative example the atlas spreadsheet of rab- 8 is shown below (Figure 21).

The overall anatomy of this mutant shows no visible phenotypic aberrations on both lightand an electron-microscopic level. The size of the mutant adult worms is similar to wild type worms. The expression of rab-8 is driven mostly in neuronal cells, as we can deduce from fluorescent studies, which might explain the absence of visible morphological defects in the other tissues. As this subcellular morphology analysis is very specific, it needs hints and assistance by other hypotheses to explain possible defects caused by the individual rab mutations. We are therefore using the results of the expression studies and experiments which assay behavior and motor programs - depicted at the bottom - to focus and guide the investigations. The mutant shows almost no aberrations in locomotion or feeding. For the defecation assay and a small but significant difference in the defecation cycle length is detectable but the expulsion efficiency is similar to wild type. This data suggests a redundant role of rab-8 in the trafficking network and that defects in rab-8 may be compensated by a second rab. From the bioinformatic analysis a candidate for this compensatory role might be rab-10. rab-10 has not been thoroughly investigated for this hypothetical role but preliminary results from double-mutant studies using RNAi procedures strengthen this hypothesis (Sasidharan, 2011).

The Wiki, upon its completion, will grant open access for all data of rab mutants and will thus allow interested scientists to compare and share their own data with the community. The Wiki is intended to function as a platform that facilitates trafficking related studies. For the future it is conceivable to incorporate also the data from RAB-effectors, SNARE or other trafficking studies. 

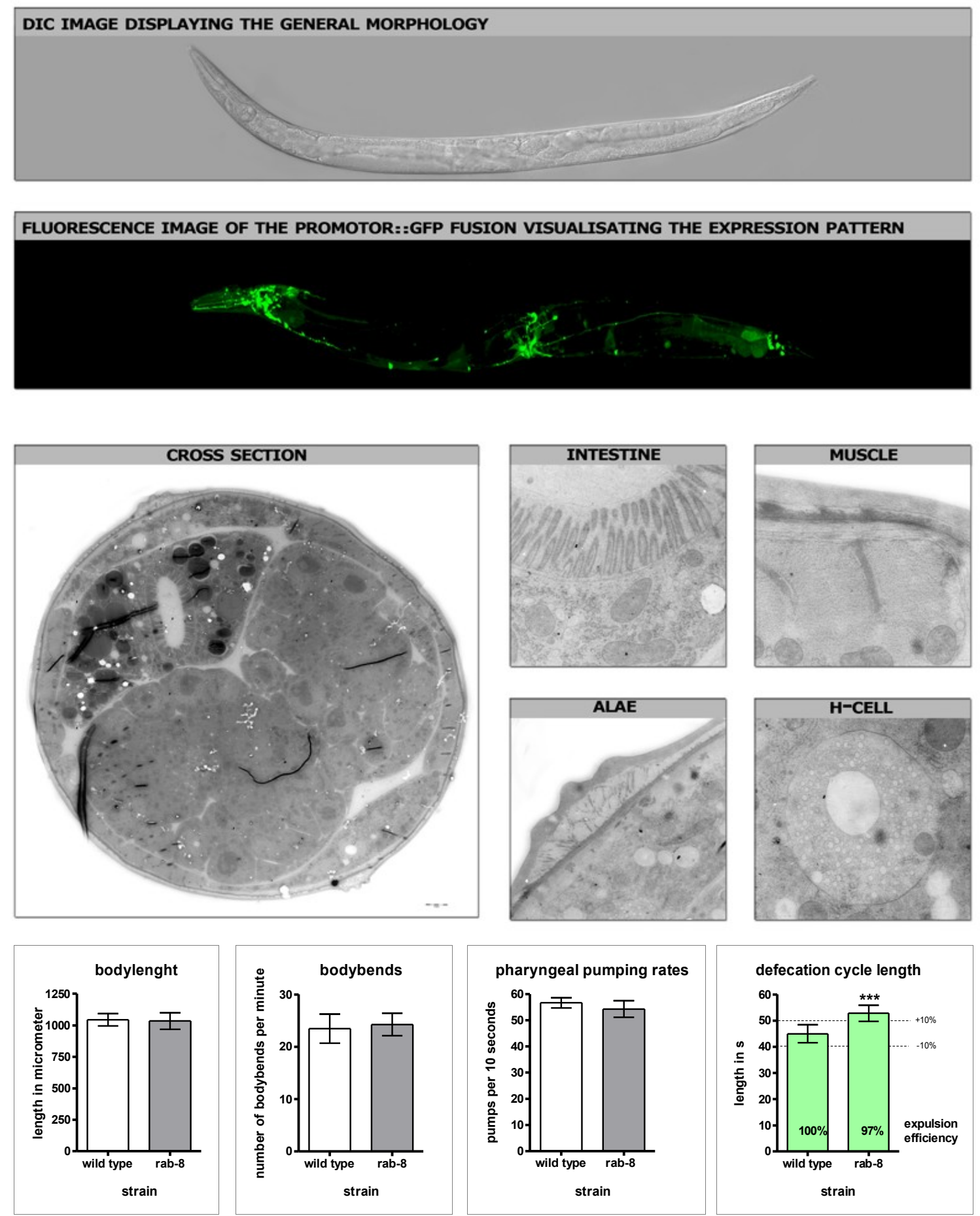

Figure 21: Wiki-Data of $r a b-8$ mutant nematodes

The morphology atlas is conceived to display general morphology of the rab mutant along with the expression pattern as shown by images from differential interference- and confocal-fluorescent microscopy (on top) and the subcellular anatomy of the worms major tissues - visualized by electron microscopy. For the rab-8 mutant general morphology appears not altered, possible due to the fact that mostly neuronal tissues express the protein.

The bottom displays the results of behavioral studies in comparison with wild type. Error Bar $=$ SD. Significance indicated by $* * *$ for $\mathrm{p}<0.001$ (t-test). 


\section{Discussion}

Here the rab family of small GTPases has been analyzed to describe the individual members of this gene family. The final goal of this investigation is a deeper understanding of the regulative roles of all Rab-GTPases in the network of intracellular transport. This broad-scale analysis uses the combined results of three individual approaches. At first, a bioinformatic analysis which examines gene- and protein sequences was initiated. Secondly, several behavioral tests which monitor motor programs and motor function were conducted. And finally, a phenotypical investigations which probes cellular- and subcellular anatomy was performed.

In a parallel investigation genetic and molecular biological test were performed by a colleague. Some of the findings of theses test are mentioned here, but for a in-depth discussion please refer to the according thesis work (Sasidharan, 2011).

\section{Bioinformatic analysis of the rab family is initiated}

To gain an overview about complexity of the rabs we have conducted a sequence based comparison of all genes, their exon- and intron-contributions and the resulting proteins sequences. From the analysis we can directly see that the worms has a very simple Rabome with low redundancy, as has been shown before (Pereira-Leal and Seabra, 2001). Protein sequence that were most identical were the pairs of RAB- 8 and RAB-10, RAB-2 and RAB-14, as well as RAB-3 and RAB-27. From the predicted 31 open reading frames (www.wormbase.org) only 28 showed detectable expression levels in a promotor::GFP assay (N.Sasidharan, 2011). The three announced pseudogenes are rab-18.1, rab-y4 and rab-y5. Interestingly the missing rab-like proteins $\mathrm{RAB}-\mathrm{y} 4$ and $\mathrm{RAB}-\mathrm{y} 5$ show high degree of similarity with RAB-2 and may represent the results of a potential gene duplications of the recent evolution. This hypothesis has not been investigated any further, but may represent a ideal situation for the study of the elevated rate of evolution that has been detected in C. elegans (Gamulin, Muller and Muller, 2000).

It may be mentioned here that in a further collaborative studies with the bioinformatics group of D. Fasshauer (University of Lausanne) we are currently performing phylogenetic studies that investigating the Rab-evolution across multiple animal kingdoms. The analysis has been started with about 20 species and 400 rabs (given by the author) and after data base searches 
with intelligent hidden-markov-model (HMMs) based routines the current numbers of species is well beyond 100 and more than 1000 rabs are integrated (internal communications). The analysis of this amount of data has however not been completed so far but is expected soon. As this group has already performed a similar evolutionary analysis of SNARE-proteins (Kloepper, Kienle and Fasshauer, 2007). A combination of the newly generated results with the previous findings might lead to an insight in co-evolution of RABs and according SNAREs - a topic of great interest for the trafficking community.

\section{Functional assays of rab mutants strongly suggest individual roles of rabs}

Several behaviors of the worms are regulated by motor programs. While body movement was only impaired in rab-2 mutants, a defect which has recently been traced to dense-core-vesicle defect (Sumakovic et al., 2009; Marza et al., 2008) the other test revealed multiple effecting rabs. Feeding is impaired in rab-2, rab-3, both rab-6 alleles and rab-10. For rab-2 (DCV defect) and the rab-3 (SV defect) the neuronal impairment already explains this effect, but for rab-6 and rab-10 this has not been investigated. In further studies we have seen a DCV defect in rab-10 mutant worms and thus a neuronal impairment may be the reason for this defect - similar to rab-2. But for rab-6 no such neuronal alterations was detected. An action in concert with eat-17 which leads to grinder defects has been reported (Anselmo, and Avery 2004). This effect is mentioned to be due to improper collagen trafficking. Grinder defect were not detected in both of the rab-6 alleles by our studies, but both mutant strains show an increased tendency to burst during adulthood. This hints towards a cuticular misstructuring and one of the main cuticle components is collagen. A weakened cuticle for rab-6 mutants was furthermore confirmed in the Hoechst staining assay, indicating that rab-6 is indeed likely to be linked to proper collagen shuttling, but not only in pharyngeal tissue as reported before but in a more tissue unspecific fashion. Further studies will hopefully determine the exact role of rab-6.

From the Hoechst staining which checks for cuticle integrity and where the rab-6 alleles showed a very strong phenotype, rab-35 showed very weak staining. rab-35 mutants was furthermore aberrant to show locomotion defect on non-seeded plates where it appeared to swim in one place rather than gliding and propelling. Both results might hint to a possible deformation of cuticular structures, such as alaes or annulies, which lessen the friction that the worms can impose on the ground. Further studies in this respect are necessary to reveal the 
exact role of rab-35 in cuticle shape and locomotion.

As a last test for motor programs the defecation cycle length and defecation efficiency of the $r a b$ mutants were analyzed. In addition to the neuronaly impaired mutants, $r a b-2$ and $r a b-3$ as well as the previously published defecation deficient mutants rab-27 (aex-6 - Thomas, 1990) several mutants were defecation deficient. Expulsion efficiency was lowered than 75 percent in $(r a b-2) r a b-7, r a b-10, r a b-19,(r a b-27) r a b-28$ and $r a b-35$. As $r a b-27$ functions to facilitate the actions of SV exocitosis it may regulate the neurotransmitter release in the neurons responsible for enteric muscle contraction EMC ( the DVB and AVL neuron - Schuske, Beg and Joergenson, 2004). Here the other rabs may function to fine tune this process but this hypothesis remains to be tested.

Prolonged cycle times were measured for (rab-3), rab-6, rab-7, rab-8, rab-18 and rab-35 while a significant reduction in cycle length was seen in rab-10 (and rab-27) mutants. This cycle is not under simple neuronal control but it is known that the defecation cycle length is controlled by a calcium transients in the intestine (Branicky and Hekimi, 2006). ITR-1 mutants, which lack the ER localized IP3-receptors, display a loss of intestinal calcium transients and show a increased cycle length. Overexpression of ITR-1, on the other hand, leads to shorter cycles (Dal Santo et al., 1999). Furthermore, it has been implicated in genes that are involved in the generation of IP3, such as phospholipase-C (PLC) are capable of regulating the cycle length (Espelt et al., 2005) . As Rab-GTPases directly interact with (phosphoinositide) kinases and phosphatases Fukuda et al., 2008) they are capable to modulate the phosphoinositol pools in the cell. It is thus possible that rab mutants change the levels of Phosphoinositol $(4,5)$ bisphosphate (PI45P2). Since PI45P2 is a precursor for the formation of IP3, changes in PI45P2 levels may indirectly affect the length of the defecation cycle though altered PI3 levels. Interestingly the mammalian orthologes of rab-3, rab-6, rab-8, and rab-35 - which are among the mutants that displayed an increase in cycle length - were shown to bind the inositolpolyphosphate 5-phosphatase (Fukuda et al., 2008).

To measure the effects of changed pools of phosphoinositols an lipid extraction (König et al., 2008) from whole worm lysates was performed by S. König in our lab. I have reprogrammed a version of my microarray analysis tool XtraCAT (Klein et al., 2009) in order to analyze the data of the lipid extraction with standard hirarchical clustering algorithms. In the preliminary and not yet robust analysis of this data we have found that altered pools of lipids (PI45P2, DAG, PIP and PI) in several rab mutants (rab-2, rab-3, rab-10, rab-11.2, rab-14, rab-18, rab-19, 
rab-27, rab-28, rab-33, rab-y3, glo-1). Unaltered level of lipids were detected for PA, PE, PC, PI3P, PI4P and rabs that not alter the lipid levels were rab-8, rab-21, rab-35, rab-38, rab-39 (wild type as reference - For specific notation please refer to the original method paper). However, this study was performed with full worm lysates a direct access to pure intestinal concentrations can be controversially discussed in respect to the results of the defecation assay. However, what we can deduce is that, RABs can indeed alter the phosphoinositol pools in the worms but further studies have to clarify their action.

\section{Tissues and cells in $2 \mathrm{D}$ and $3 \mathrm{D}$}

In addition to the functional analysis we classified the rab mutants morphologically. As rabs show tissue specific expression patterns and since RABs show specific subcellular localization in C. elegans (Sasidharan, 2011) an analysis of tissues and subcellular compartments was necessary in order to classify the mutant strains. This EM based study used the near-native preservation method of high pressure freezing in combination with slow substitution embeddings in order to accurately reveal the architecture of the different tissues and subcellular compartments in worms.

From the comparison with a data set for wild type it became obvious that no major defects are detectable for the rab mutants. This is a preliminary result since the study has analyzed only one third of the mutants so far but we expect no major aberration in the rest of the mutants too. The result of this study are combined in a so called Rab-Atlas. We hypothesize this due to the fact that several of the RABs share a high similarity in structure. By cooexpression of these pairs in the same tissue they may thus compensate for each others loss. Furthermore this option allows a more distinct fine-tuning of certain RAB actions through rab-cascades. Experiments that perform quasi-double-knockouts of rabs, by using mutants strains and RNAi, have already lead to the finding (among others) that $r a b-8$ and rab-10 are indeed synthetically lethal (Sasidharan 2011). Note here that the close interaction of both genes was already postulated by the bioinformatic analysis. This demonstrates that the trafficking machinery is build very robust and that the loss of one rab can be compensated in a redundant fashion by other rabs. The rabs that are canonically (alone) lethal the bioinformatic analysis showed large divergence towards the other genes. The action of these rabs seem to allow no alterations. The rab-7 mediated protein degradation or the rab-5 mediated endocytosis (Stenmark, 2009) 
illustrate this idea. In higher organisms the Rabome is often more complex as for example humans were over 60 rabs are present. rab-3 is present in four alleles in the human system and its action on SV tethering is still conserved. Here we speculate that only the presence of multiple alleles opens the opportunity to proper finetuning in the numerous different neuronal cell types.

Because no alterations were visible on cellular level we focused on the mapping of intracellular compartments with a special focus on the Golgi, as it represents the most central trafficking organelle. While reconstruction from serial sections was good enough for tissue reconstructions we had to turn to the tomographic approach to directly resolve the 3D architecture of the Golgis. In this study we show that the Golgis are not similar to each other but show a tissue and cell specific shape. This shape most likely resembles the functional states of these Golgis. While muscular Golgis seems to be small present and present in moderate numbers the intestinal Golgis are swoolen and present very noumerous. Additionaly we find that both tissues evenly distribute the Golgis in their cytoplasm. This is indicating that active muscle Golgis are needed less for the maintance of proper muscle function while the intestinal Golgis are of huge importance to the proper functioning of the intestine. Similarly the coelomocytic Golgi are centralized in a block of Golgis to form a super-Golgi to maintain the heavily active endocytotic action of these scavenger cells. In other systems the merging of Golgis has been reported in similar situations, a mechanism that seems not to have evolved in the nematode system. The orientation and location of Golgis seems thus to be of great importance. A very intuitive example for this is given for the long and polarized neurons. Here only two large Golgis are located adjacent to the nucleus of the cells, one pointing into the direction of the axon the other one to the neurite. For the synaptic regions of the neurons no further Golgis are needed as these regions remain to be functional with the help of proper vesicle recycling mechanisms. Since only a few DCV have to be replenished this could explain why only two Golgis are present in neurons. In heavily active tissues such as embryos and hypodermal cells (which massivly secrete the cuticle components that are necessary for the subsequent larval molds and their associated cuticle regenerations) the very abundant Golgis a very large and consist of multiple stacks this also reflect the highly active nature of these tissues. As our preliminary study has revealed these distinct Golgi-shapes we are currently investigating the rab mutants that were shown to partially or fully colocalize to golgi markers. The three-dimensional investigation which has been set up for this purpose will help to resolve 
the pleomorph architecture of these Golgis in detail.

In addition to the subcellular characterization of Golgis we have focused on the subcellular anatomy of the neuronal en-passant synapses of the worm because many rabs show strong neuronal expression patterns (Sasidharan). The general nature of this process is individually presented and discussed in the next chapter together with the tool that has been developed to perform the actual synaptic comparisons. For further details the reader may thus refer to the next chapter. It however remains to be briefly mentioned that only the high-pressure freezing method could preserves the ultra-fast trafficking events in those synapses. This method is thus of the most central importance to the entire rab classification.

\section{The open access Rab-Wiki}

In order to accurately display and combine the plethora of results of the performed studies of N.Sasidharan, the author and other coworkers and collaborators I have furthermore designed a Wiki-based-platform that contains all generated information in an intuitive fashion (www.enig.de/wiki_seimer).

As we have already seen in our study, the individual test may not characterize novel phenotypes. However, the integrative merit of all test is far more conclusive than the pure sum of the individual results. And, since the ultimate goal of this study is to lay the basis for a fundamental trafficking atlas, this Rab-Wiki probably represents the biggest achievement of this thesis work. This open access Rab-Wiki will, upon full completion of all experiments, function as the basis for the trafficking community in C. elegans and all other fields of science. It is intended to facilitate the propagation of trafficking based knowledge and experiences and it will hopefully help to foster future collaborations. If this venture succeeds an extension to other species of the worm and other model organisms is possible just like a connection of this Rab-Atlas into the already existing databases Worm-Atlas (www.wormatlas.org) or Worm-Base (www.wormbase.org). 


\section{Chapter IV. The image analysis tool XtraCOunt}

\section{Introduction}

\section{Neuronal trafficking is RAB-dependent}

In recent studies we found that the majority of rabs are expressed in the nervous system (Sasidharan, 2011). The nervous system consists of cells that are highly polarized cells, with highly specialized membrane domains. The membrane trafficking in neurons involves transport of proteins necessary for synaptic transmission and remodeling, as well as lipids necessary for neurite growth. Since neurons have their unique cellular morphology and their specific trafficking requirements the assumption that neurons use the same secretory machinery as the other eukaryotic cells has been challenged. The role of Rab-GTPases in several aspects of membrane trafficking has thus been analyzed in detail for neurons. RabGTPases have been shown to be involved in neuronal development (Di Giovanni et al., 2006), endocytosis and retrograde transport (Deinhardt et al., 2006). At synapses, Rab-GTPases perform specific functions in synaptic vesicle (SV) exocytosis and receptor recycling. An involvement of $r a b-3$ in parallel with rab-27 for tethering of SVs to the presynaptic specialization and also in the final stage of exocytosis has been well established (Mahoney et al., 2006). Interestingly, a recent proteomic analysis of highly purified SVs revealed that, in addition to RAB-3 and RAB-27, a large set of other Rab GTPases can be copurified with SVs (Takamori et al., 2006, Pavlos et al., 2010). This suggests that multiple Rab-GTPases might be needed for correct SV-trafficking and regulated release of SVs. Although some of these rabs have been functionally characterized in non-neuronal cells, their specific roles in neuronal membrane trafficking remain to be determined and for this purpose we started to study synaptic transmission at the ultrastructural level in C. elegans.

\section{The neuromuscular junction is a model system for synaptic vesicle release studies}

The neuromuscular junction of $C$. elegans is a well- studied and well- described model for studying the synaptic transmission and neuronal function. The 302 neurons of the worm are 
invariant in location and connectivity, and each has been mapped by electron microscopy (White et al., 1986). The neuromuscular junction of C. elegans is morphologically different from the mammalian junction in the sense that the muscles, instead of the neurons, send projections to the nerve cords which present "en passant" synapses to muscle arm (compare Figure 18). Similar to the mammalian system, the contraction and relaxation of body wall muscle is controlled by the two neurotransmitter systems: acetylcholine and GABA. By alternating the contraction and relaxation of opposing body wall muscles, sine wave locomotion propels the worm forward.

In motorneurons, neurotransmitters are packaged and released from SVs. These vesicles dock at the active zones, where they subsequently undergo a priming step, an event which prepares the SVs to respond to the calcium influx through voltage gated calcium channels, which are activated by depolarization of the neuron via an arriving action potential. Upon calcium influx, SVs fuse to the plasma membrane and release their neurotransmitter into the synaptic cleft. After complete fusion, vesicle proteins and membrane are retrieved by clathrin-mediated endocytosis. In the lumen of the synapse, these retrieved vesicles are recycled and refilled, therefore ready for another cycle. The nature of this cycle has been thoroughly investigated in recent years (reviewed in Südhof, 1995, 2000, 2006 ) but although the involvement of rabs in docking, priming and fusion has been reported, the exact molecular integration of all rabs in this mechanism is not fully understood.

\section{XtraCOunt is a versatile image analysis tool}

Neuromuscular junctions have been studied in various model organisms to investigate the molecular machinery that regulates synaptic the vesicle exocytosis. Such experiments are conducted by the analysis of the vesicle distribution within synaptic terminals of electron micrographs in cross-sectioned synaptic terminals. On these micrographs, the distance of each vesicle to the presynaptic density is measured. Vesicle-distance histograms can be thus be plotted to reveal changes among wild type and mutant vesicle distributions. Furhermore, in order to measure the size (area) of objects, the micrographs can be cut out and weighed on fine balances while circumferences of object were traditionally measured using string length, which is wrapped around pin needles that were previously stuck into the trace of the object on the micrograph. Thus, in the past, the analysis of synaptic vesicle distributions were performed 
manually - a time-consuming, labour-intense and thus error- prone task.

To decrease the human bias of this method, we developed a semi-automated image analysis tool XtraCOunt which facilitates the analysis of synaptic vesicle distribution studies with electron micrographs (Figure 22A). The program greatly enhances the speed of the analysis and automatically exports data sheets for all measured features to enable data processing with statistical software. Although designed to aid Caenorbabditis elegans studies, it can also be used for neuroscientific studies of other model organisms like Drosophila melanogaster or Mus musculus. Additionally, to offer enhanced readouts, previously not accessible by the manually performed studies, we included automations that enable focusing on specific vesicle populations in the periactive zone of synapses (Figure 22B) and we integrated features to measure linear- and non-linear distances (Figure 22C\&D) and also to enable vesicle clustering studies. Although XtraCOunt is generally conceived to facilitate vesicle distribution studies, it is actually a more widely applicable toolbox due to the variability of measurements achievable. It can be applied to image analysis studies in other scientific fields, as we show here for vesicular size analysis or nematodes growth assays. The following chapter introduces the general features of the program and demonstrates how XtraCOunt helps to quickly produce more reliable and less biased results. 
A

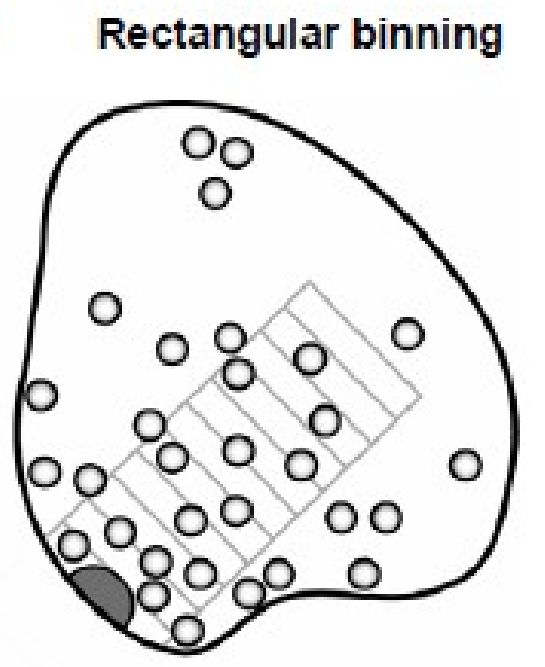

C

\section{Linear distance measurements}

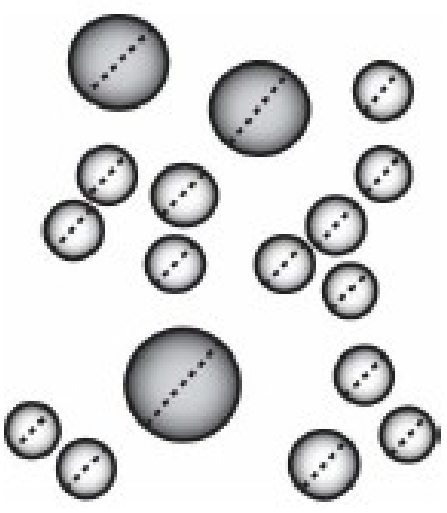

$\mathbf{B}$

Periactive zone readout

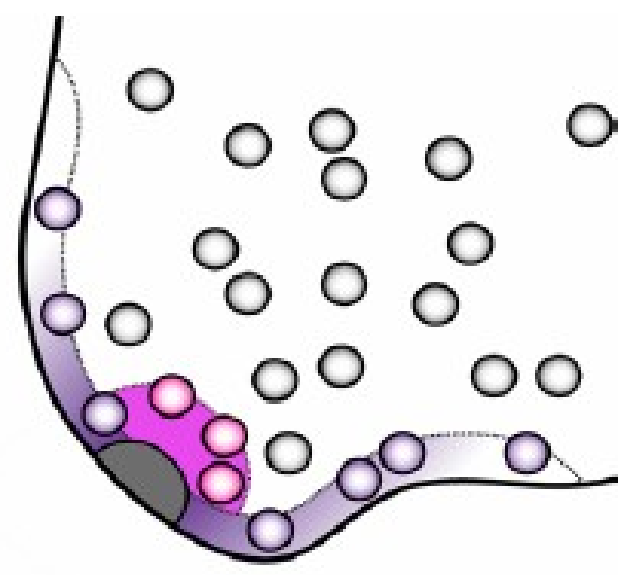

D

Irregular distance measurements



Figure 22: XtraCOunts novel features and versatility

(A) XtraCOunt enables the analysis of vesicle distributions in synaptic nerve terminals. To account for the mostly irregular shaped nerve terminals of nematodes, an optional, novel rectangular grid analysis was integrated into the program. (B) The user is furthermore enabled to monitor the amount, position and thus gradients of the subpopulation of vesicles located in the periactive zone of the nerve terminals. As additional features the user can perform distance measurements along with the distribution analysis. (C) Linear distances of vesicle diameter or (D) irregular distances like nematodes lengths can therefore be both be measured with XtraCOunt.

\section{XtraCOunt supports vesicle distribution studies}

The analysis of a vesicle distribution in nematode neuromuscular junctions (Figure 23A) with the help of the program XtraCOunt consists of four basic steps (Figure 23B). 


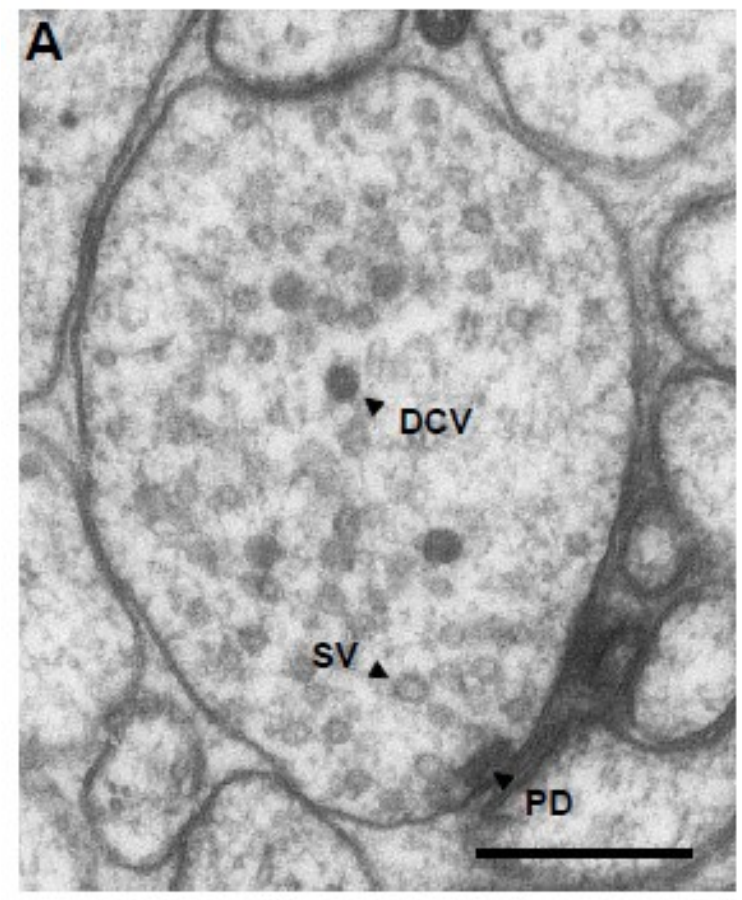

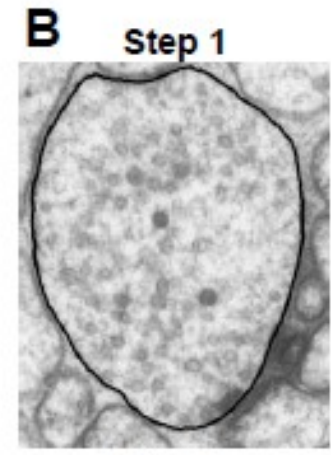

Step 3

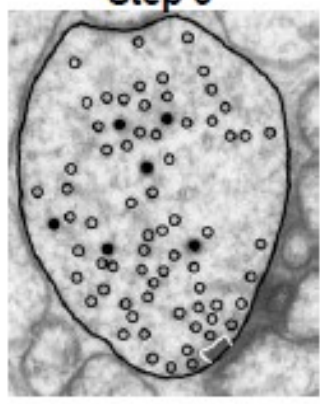

Step 2

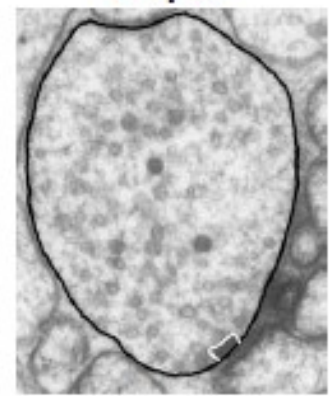

Step 4

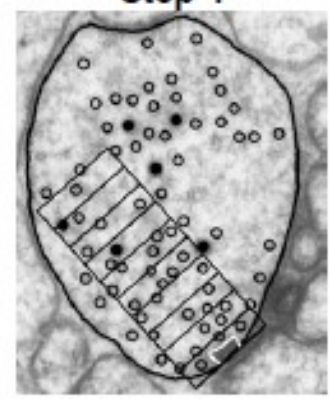

Figure 23: Individual steps of a vesicle distribution analysis with XtraCOunt.

(A)Original electron micrograph showing a 50nm cross section of a C. elegans neuromuscular junction. PD Presynaptic Density, SV - Synaptic Vesicle, DCV - Dense Core Vesicle. Scale $=200 \mathrm{~nm}$. (B) Stepwise analysis procedure in XtraCOunt. Step 1 - Loading the micrograph, scaling and encircling the plasma membrane of the presynaptic terminal. Step 2 - Circumscribing of the intracellular border of the presynaptic density. Step 3 Marking of vesicles in the terminal. The user can differentiate SVs and DCVs. Step 4 - Output data is generated with standard settings automatically. Optionally a rectangular grid can be added to bin the distribution relative to the PD.

In the first step, after loading, labeling and scaling of an image, the user encircles the plasma membrane of the synaptic terminal. The program functions to record all pixel coordinates that were labeled as a membrane point. The enclosed surface area of all these point is displayed after the user is finished with the assignment. This calculated value can be used for size normalization purposes. It can be useful for comparing total vesicle counts of terminals with different sizes. In case of a mitochondrion or other large organelle being present in the terminal, the user able to subtract up to one large ellipsoid shaped structure from the total surface area.

In the second step the user circumscribes the internal boundary of the heavily stained presynaptic density and again all the coordinates of each pixel are stored for this object. In the third step of the procedure all synaptic vesicles and dense core vesicles are assigned by the user by simply clicking in the middle of the vesicle on the micrograph. The membrane and density points together with the stored coordinates for each vesicle are the basis for all later 
calculations. XtraCOunt calculates the minimal, average and maximal distance towards the plasma membrane and the presynaptic density automatically for each vesicle.

XtraCOunt calculates summary reports in addition to the raw data. The fully automatic procedure calculates a heuristic report displaying the total vesicle counts located in circular bins around the presynaptic density. The size of the bins is defined by the user. For a rectangular binning strategy the user has to set the position of a grid in an optional acquisition step. For a more detailed description this procedure is included in the manual of the program in the supplementary information. The manual includes technical and algorithmic information, hints and caution warnings and also presents a sample work flow of XtraCOunt. 


\section{The program features of XtraCOunt are designed to be intuitive}

The program XtraCOunt is packed into a single file which is executable with any standard IBM computer operating a Windows platform. No additional software is needed to install the tool. The graphical user interface (Figure 24A) is structured in an intuitive top to bottom fashion to

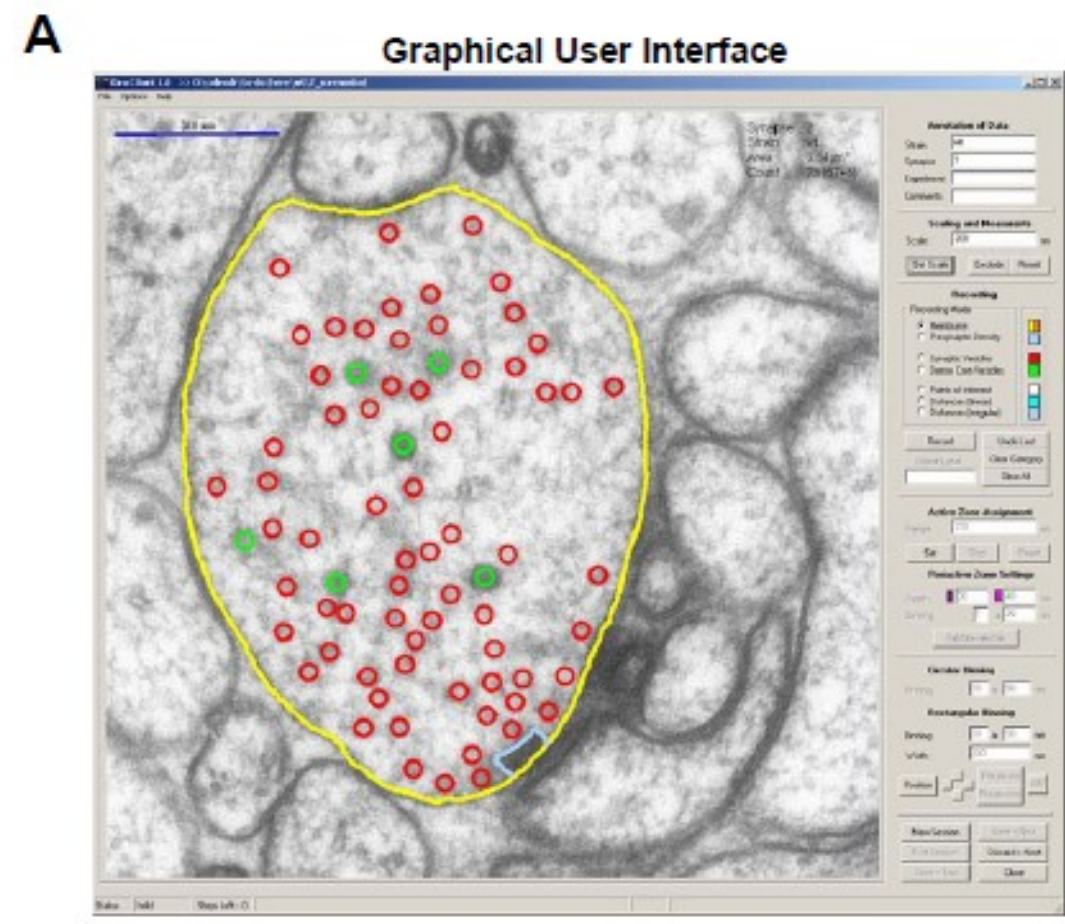

B

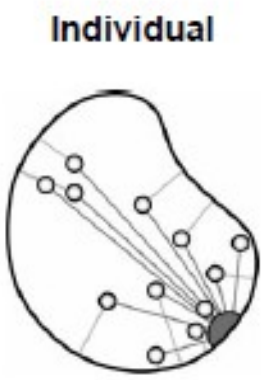

Supported analysis strategies
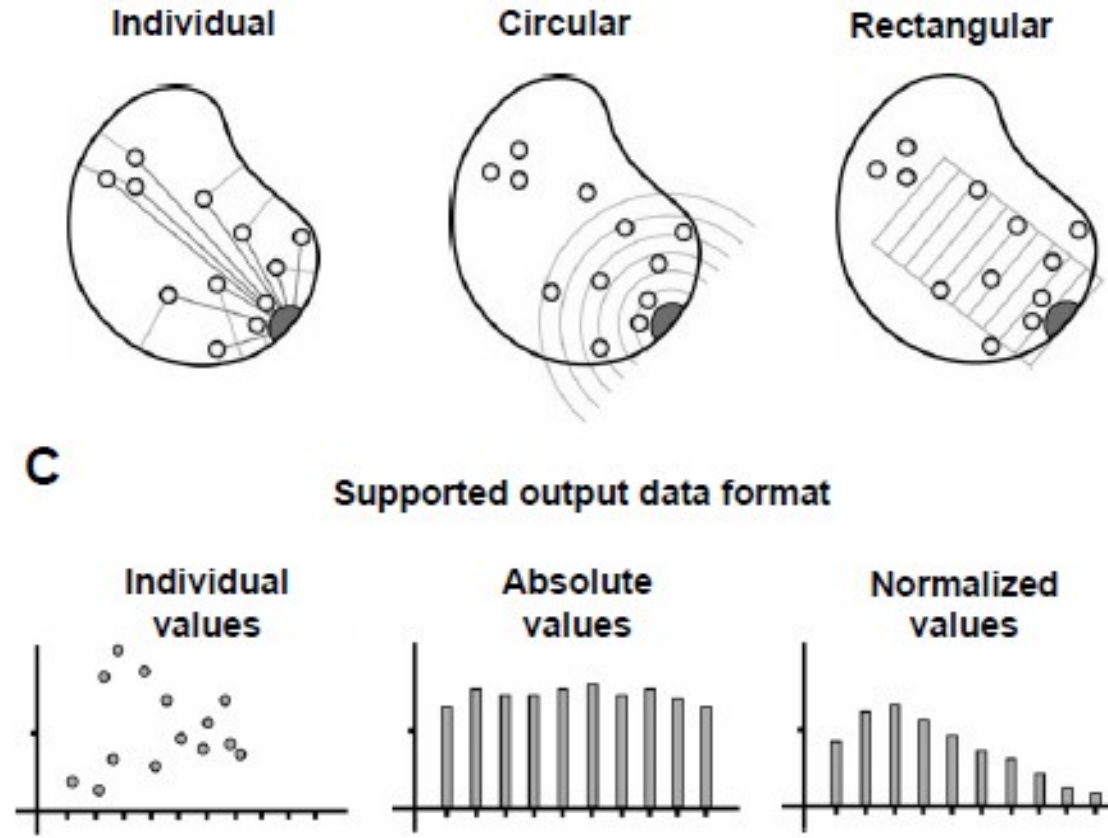

Supported output data format
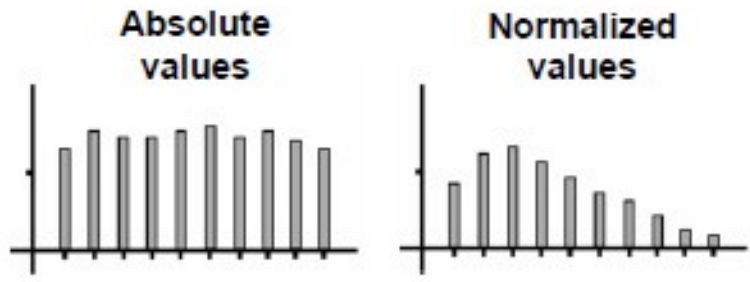

Figure 24: XtraCOunt automates the classical analysis procedure.

(A) The User interface of XtraCOunt is easy to use and arranged in an intuitive fashion in order to empathize the individual steps of the manual analysis procedure. The user can choose among different analysis methods (B) and a comparison of these methods is possible with the help of different automatically generated output data formats $(\mathrm{C})$. 
enable use without previously acquired computing skills.

XtraCOunt supports the analysis of vesicle distributions using three different strategies (Fig. 24B). Vesicles can be analysed individually or in the classical circular heuristic fashion. In addition to the standard methods a novel rectangular heuristic can be used.

All data is finally exported in the form of summary reports along with all raw data. The use of tab-delimited text files ensures that output files can be imported without conversion problems into standard statistical software like Excel, Matlab or R. Three individual outputs are generated by XtraCOunt (Figure 24C). All the raw data is exported. For each vesicle, SV or DCV, all distance parameters are listed in a table. This includes the distances to the membrane, the PD and the AZ. A second table comprises the counts of vesicles located in circular bins around the active zone and a third table shows a summary for the optional rectangular grid binning analysis.

These three tables get stored in individual reports for each image along with the original picture and the final GUI screenshot, enabling a retracing of every single measurement. In order to facilitate the analysis of multiple images data-combining files are exported at the end of each session. The user has thereby a quick access to any desired data subsection.

\section{Analysis of distribution patterns in different model organisms with XtraCOunt}

The tool XtraCOunt was designed to be a robust aid that facilitates and standardizes vesicle distribution studies. It was developed to help analyse the irregular shaped synaptic nerve terminal of the nematode C. elegans, D. melanogaster and $M$. musculus. Therefore the program offers all classical methods of the standard (manual) analysis procedure. rab-3 mutant nematodes are defective in vesicle tethering to the PD. The tethering defect is already visible by eye on electron micrographs when comparing wild type (Figure 25A) and rab-3 mutant terminals (Fig 25B). This defect has been well studied and characterised in the nematodes and other model systems in the previous years (Nonet et al., 1997; ). 
When analysing the effects of a disruption of the protein with XtraCOunt we can recapitulate these findings (Figure 25C). rab-3 mutations cause synaptic terminals to exhibit a clear
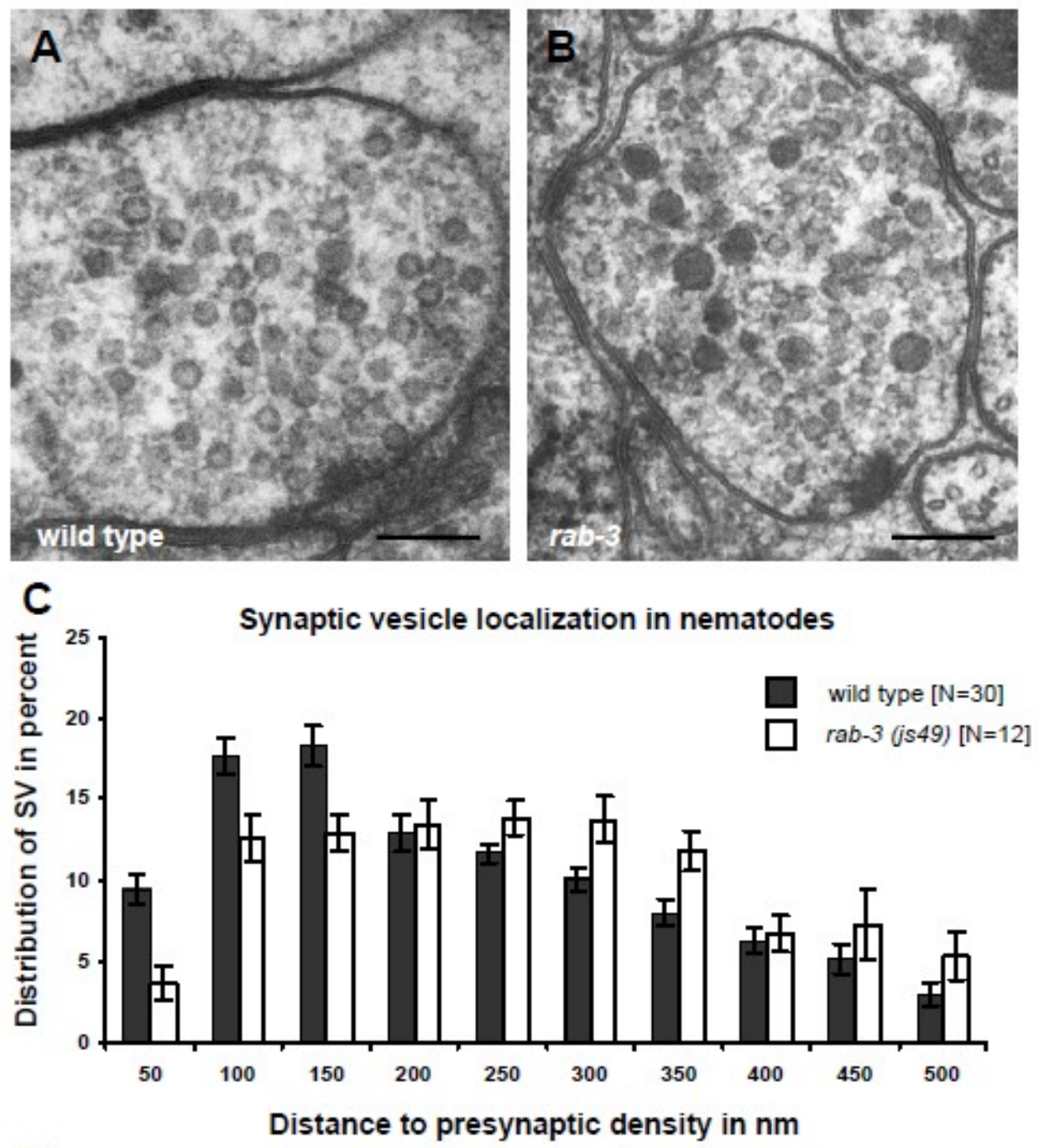

\begin{tabular}{|l|c|c|c|c|c|c|}
\hline \multicolumn{1}{|c|}{ Strain } & $\begin{array}{c}\text { Average } \\
\text { cell area } \\
\text { in } \mathrm{nm}^{2}\end{array}$ & $\begin{array}{c}\text { Average } \\
\text { SV count } \\
\text { per profile }\end{array}$ & $\begin{array}{c}\text { Average } \\
\text { SV diameter } \\
\text { in nm }\end{array}$ & $\begin{array}{c}\text { Average } \\
\text { DCV count } \\
\text { per profile }\end{array}$ & $\begin{array}{c}\text { Average } \\
\text { DCV diameter } \\
\text { in nm }\end{array}$ & N \\
\hline wild type & $0.24 \pm 0.03$ & $35.4 \pm 2.4$ & $27.6 \pm 0.9$ & $2.0 \pm 0.2$ & $45.2 \pm 1.1$ & 30 \\
\hline rab-3 (js49) & $0.23 \pm 0.03$ & $37.3 \pm 3.4$ & $27.8 \pm 0.7$ & $3.2 \pm 0.5 *$ & $41.5 \pm 1.1 *$ & 12 \\
\hline
\end{tabular}

Figure 25: Analysis results of rab-3 mutants

EM pictures of $50 \mathrm{~nm}$ thin cross sections of motoneuron presynaptic terminal of (A) wild type and (B) rab-3(js49) mutant animals, defective in tethering to the PD. Scale Bar $=50 \mathrm{~nm}$. (C) The average percentage of vesicles found in $50 \mathrm{~nm}$ bins is plotted against the distance from the electron-dense presynaptic density. Synaptic vesicles were counted and mapped for rectangular 500nm long grids with XtraCOunt. (D) XtraCOunts additional readout from the acquisition procedures. The tool enables to measures cell sizes, track the count for individual vesicles pools and supports linear as well as nonlinear distance measurements. Error \pm SEM. Statistical differences marked with $*$ if $\mathrm{p}<0.05$. 
tethering defect by showing a distribution of vesicles with no gradient towards the PD. Along with the gradient analysis we were able to show that the synaptic terminals in nematodes are comparable in size and SV abundance (Figure 25D). However, the amount of DCV is slightly increased accompanied by a slight decrease in size of these DCV. The defect of tethering strong enough to affect the membrane associated vesicle pool in rab-3 mutant (Figure 26).

A



B

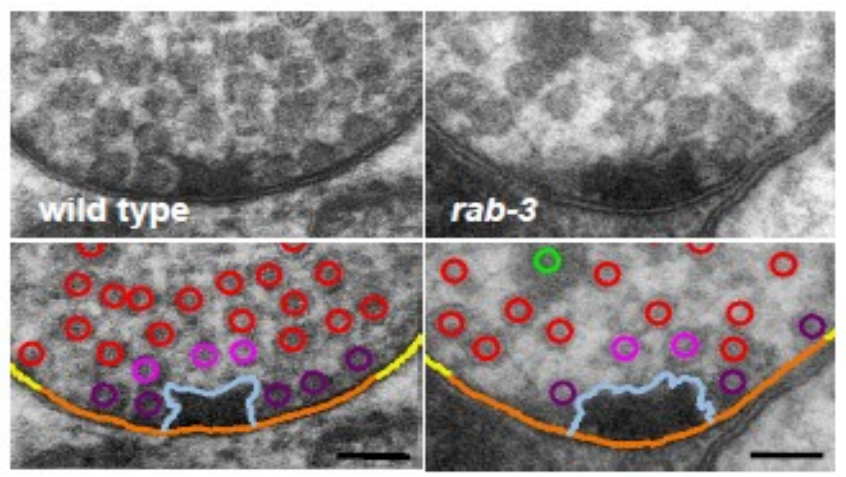

C

Synaptic vesicle localization in the periactive zone of nematodes

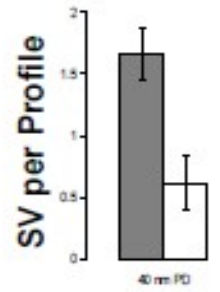

0

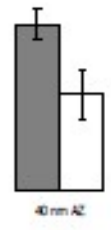

I

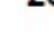


Apart from adjustments made to apply this tool for C. elegans we have maintained the all steps for a standard analysis procedure. An investigation of synaptic terminals of wild type $D$. melanogaster (Figure 27A) shows that this is standard procedure (Figure 27B) and displays the acquired data in all standard output formats (Figure 27C) of XtraCOunt.
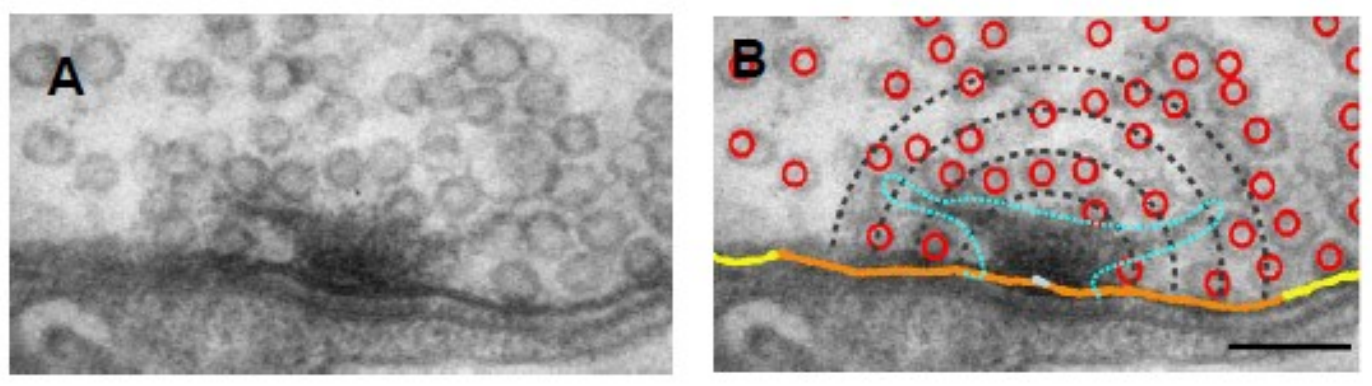

C

Synaptic vesicle localization in flies

Individual distance measurment

Circular binning heuristic

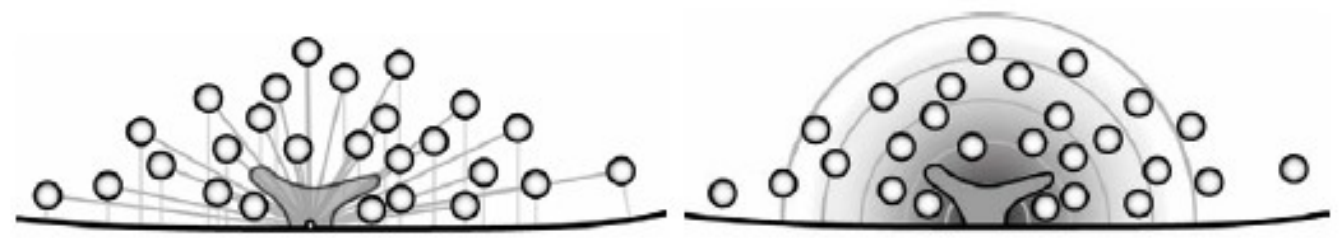

Cloud diagram

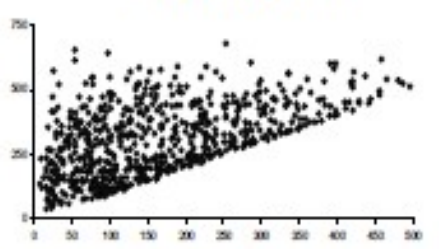

\section{Total count}


\section{Distance to presynaptic density in $\mathrm{nm}$}

Figure 27: Synaptic vesicle localization in drosophila.

(A) Vesicle distribution at neuromuscular junctions in D. melanogaster. Serial section electron micrographs of neuromuscular junctions of wild-type fly larva, were (B) analyzed using XtraCOunt. Synaptic vesicles were counted and mapped individually as well as for circular grids (virtual bins are indicated as dashed, black lines). Section thickness $=60 \mathrm{~nm}$. (C) The vesicles distances are plotted and analyzed with different methods. The cloud diagram presents the raw data information depicting each vesicles distance to plasma membrane and presynaptic density. For binning strategies one can use the total counts approach. All vesicles found in $50 \mathrm{~nm}$ bins are plotted against the distance from the root of the presynaptic density. Alternatively the values of the each bin can be normalized to compensate for the increasing size of radial bins (normalized count). The output format can be specified by the user to account for their individual needs. Error bars \pm SEM. 
When analysing the synaptic function the size of the synaptic vesicles is often at the focus of vesicle-related studies. These distance measurments can also be performed with XtraCOunt too. In previous studies mutations affecting the protein FAT-3, a fatty-acid desaturase, have been traced to affect the size of SVs (Marza et al., 2008). When compared to wild type (Figure 28A) nerve terminals of mutant fat-3 nematodes (Figure 28B) show a clearly visible size increase of synaptic vesicles. Here we show that with XtraCOunt we were able to recapitulate the findings of increased SV diameters for fat-3 mutant nematodes. The entire population of synaptic vesicles is shifted in size in fat-3 mutants (Figure 28C). As this analysis can now easily be integrated into any study we are optimistic that subtle changes can not be overlooked.

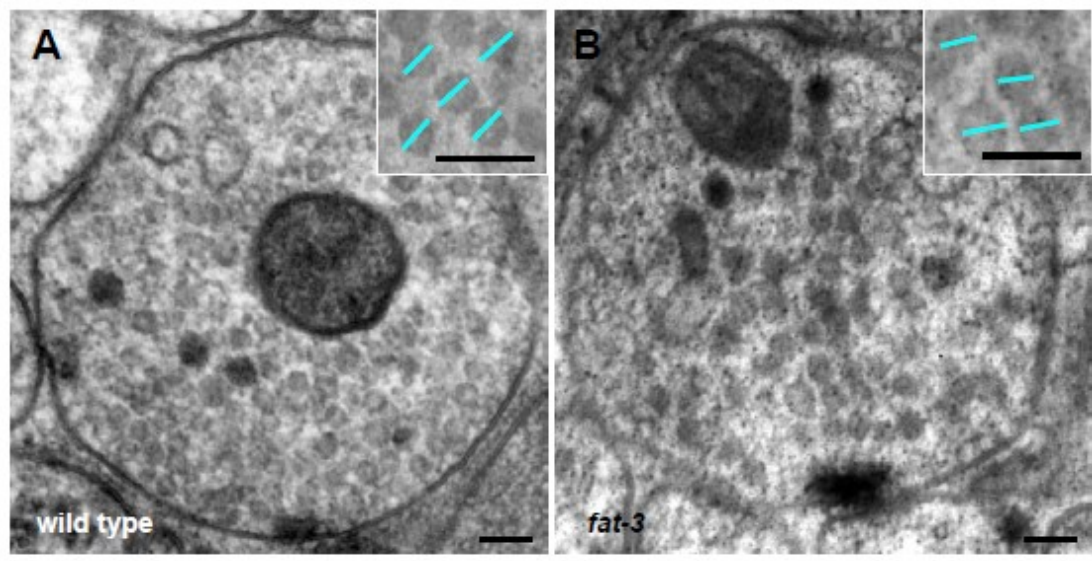

C

Synaptic vesicle size variety in nematodes

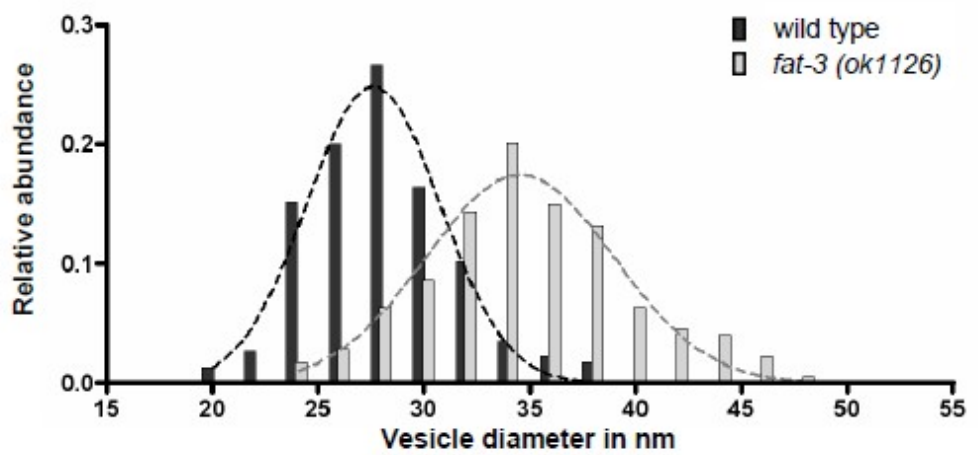

Figure 28: Linear distance measurement tool of XtraCOunt.

$50 \mathrm{~nm}$ thin section electron micrographs depicting the synaptic vesicle pools for (A) wild-type and (B) fat-3 (ok1126) mutant nematodes were examined. Insets magnify representative vesicles measured with XtraCOunt. Scale Bar $=50 \mathrm{~nm}$. (C) The diameters of individual vesicles are plotted against the frequency of their occurrence. A gaussian fit (dashed line) approximates the maximum of occurrences for wild type or mutant vesicle size at $27.6 \mathrm{~nm}$ and $34.5 \mathrm{~nm}$ respectively.

In contrast to this simple diameter calculation, which is performed with a straight line connection of two points, a second distance measurement tool is enhancing the versatility of 
XtraCOunt. The user is offered to trace individual splines in an image. The lengths of the acquired splines are then calculated by XtraCOunt. This feature can be used to measure circumferences of irregular shaped entities such as Golgi cisternaes or lengths of pleomorph objects such as axonal neurite outgrowths.

To demonstrate this feature we classified the size of nematodes suffering from a growth and shape affecting mutation of $d p y-20$ in C. elegans. When comparing synchronised growing adult nematodes the dumpy phenotype is obvious for the dpy-20 mutant worms (Figure 29A). Analysing the body length of the nematodes with the irregular distance measurement feature XtraCOunt, we are now able to accurately quantifies this finding (Figure 29B). For irregulardistance measurements subtle changes will thus be easily monitored using XtraCOunt.
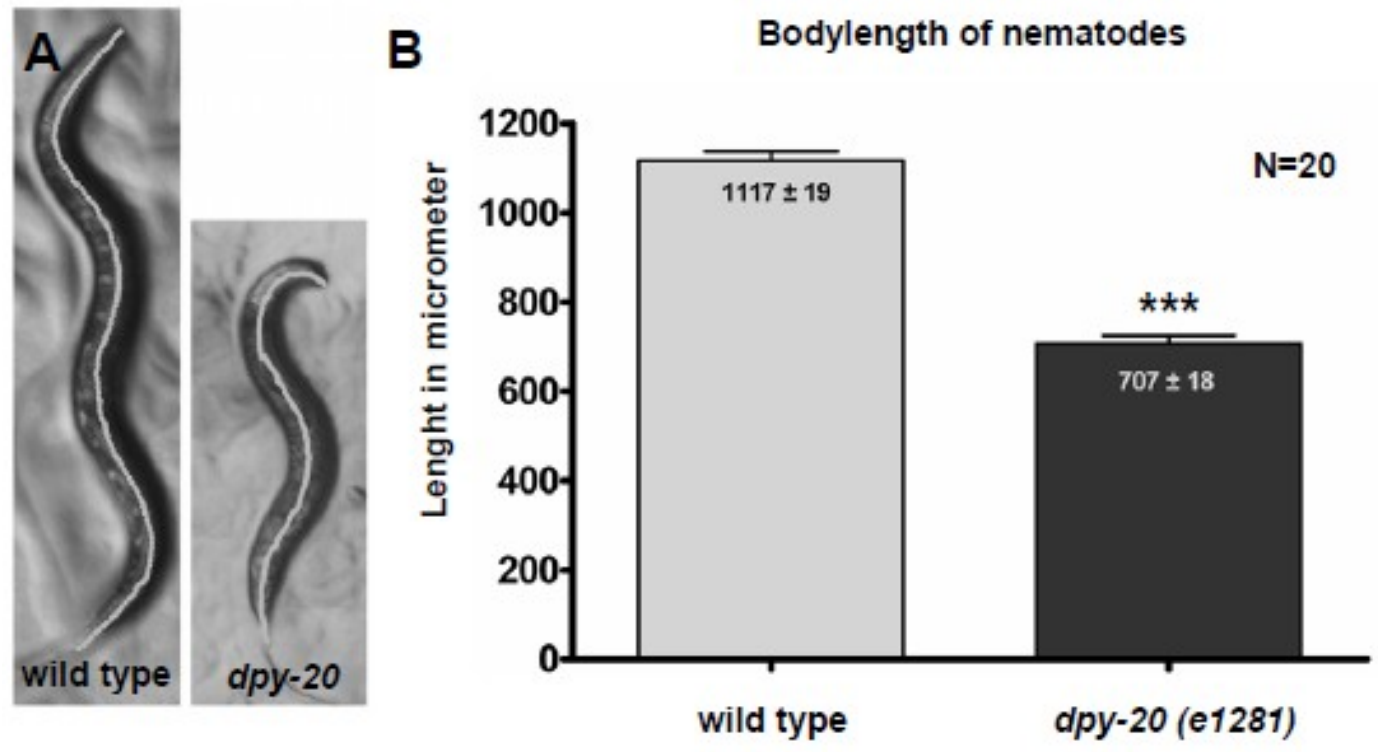

Figure 29: Nonlinear distance measurements with XtraCOunt.

(A) Representative nematodes (scaled equally). (B) Body length of adult nematodes for wild type and dpy20 (e1281) mutant animals are analyzed with XtraCOunt. $\mathrm{N}=20$. Error bar $=$ SEM. Statistical significance of student $\mathrm{t}$-test for $\mathrm{p}>0.001$ is indicated by $* * *$. 


\section{XtraCOunt enhances the speed of rab-10 vesicle distribution studies}

Having shown that all features of the novel program can assist imaging and vesicular distribution studies the program was then tested in the analysis of the most promising candidate of the Rab-Atlas studies. rab-10 mutans revealed several shortcoming in the neuronally triggered behavioural test but showed no obvious cellular- or subcellular morphological defect (apart from the appearances of gut granules in large amounts). Based on highly suggestive preliminary results from our Rab-Atlas studies and the localization results (Sasidharan, 2010), we initiated detailed rab-10 analysis on synaptic level to look for a possible defects that would explain the obvious shortcomings of rab-10 mutants in most of the motor programs.

\section{Dense-core vesicles in rab-10 mutants show reduced size}

The general morphological appearance of synapses appears wild type like in rab-10 mutant strains (Figure 30A). The overall distribution of of synaptic vesicles (SVs) is not altered (Figure 30B) and their size is conform with wild type SVs. (Figure 30C and D). However, the rab-10 mutants show slightly smaller dense core vesicles (DCVs) (Figure 30C and E). Further investigations to clarify the novel implication are currently trying to determine the exact involvement of rab-10 in DCV biogenesis or maturation. Ongoing studies suggest and involvement of rab-10 endocytic recycling of vesicles, but for further information please refer to the results of my colleague Nikhil Sasidharan who apart from the molecular biological aspects of the Rab-Atlas focused his work on rab-10 (Sasidharan, 2011).

With the help of XtraCOunt this analysis of 50 synapses was performed within several hours while the manual procedure would have taken days. In addition the subtle change of densecore vesicle size might not have been traced if manual measuring strategies would have been used. 

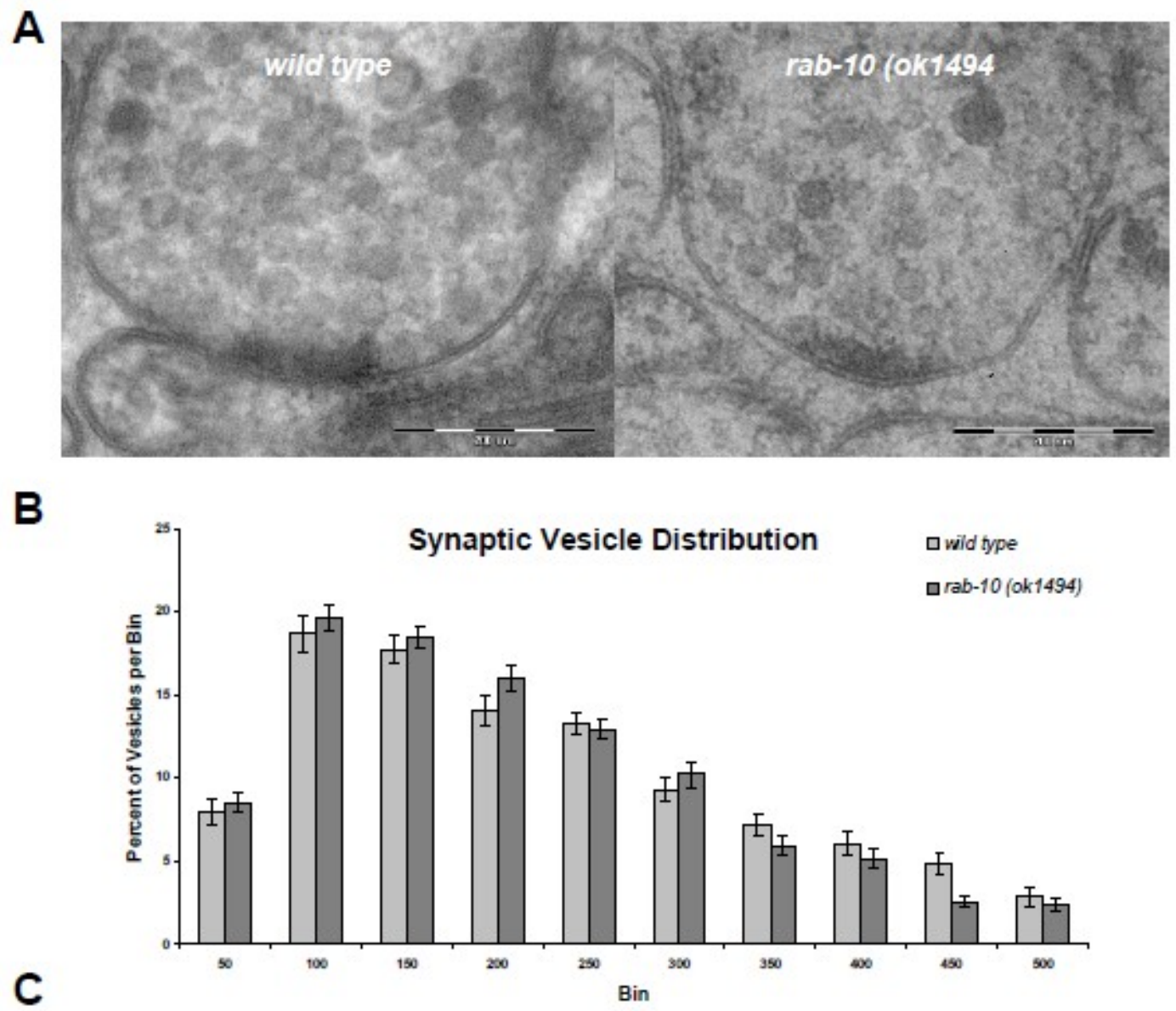

\begin{tabular}{|c|c|c|c|c|c|c|}
\hline Genotype & $\begin{array}{c}\text { Pronles I } \\
\text { animals }\end{array}$ & $\begin{array}{c}\text { Average synaptic } \\
\text { Area } \\
\text { I pronle }\left(\mathrm{\mu m}^{2}\right)\end{array}$ & $\begin{array}{c}\text { Average } \\
\text { sV number I } \\
\text { proflite }\end{array}$ & $\begin{array}{l}\text { Average } \\
\text { SV dlameter } \\
\text { (nm) }\end{array}$ & $\begin{array}{c}\text { Average } \\
\text { DCV number } \\
\text { I pronle }\end{array}$ & $\begin{array}{c}\text { Average } \\
\text { DCV dlameter } \\
\text { (nm) }\end{array}$ \\
\hline wild type & $53 / 8$ & $0,24=0,03$ & $33,4 \pm 2,2$ & $27,6 \pm 0,3$ & $2,1 \pm 0,2$ & $45,26 \pm 0,7$ \\
\hline $\operatorname{rab}-10(0 k 1404)$ & $51 / 7$ & $0,21=0,04$ & $31,2 \pm 1,9$ & $27,9 \pm 0,3$ & $1,9 \pm 0,2$ & $41,39 \pm 0,4$ \\
\hline
\end{tabular}

D



E

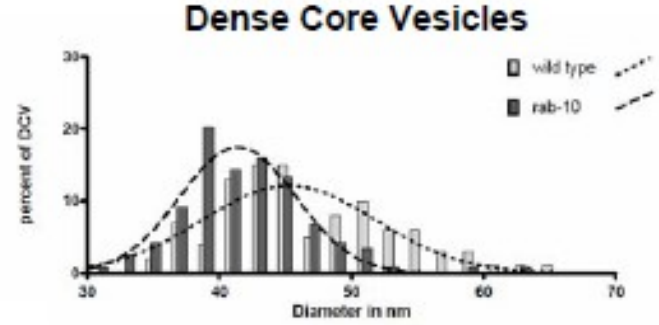

Figure 30: Ultrastructual analysis of rab-10 mutant synapses

A) TEM images showing the wild type like appearance of $r a b-10$ mutant synapses. Scale bar $=500 \mathrm{~nm}$. B) The synaptic vesicle distribution profile demonstrates no aberration in rab-10 mutants. Error bar $=$ SEM. C) The statistics from XtraCOunt reveal a DCV size defect in the Rab-10 mutants. D) The entire population of SVs shows similar size distributions for rab-10 mutants and wild type. E) The size distribution of DCVs is shifted towards smaller vesicles in rab-10 mutants. (dotted lines $=$ gaussian fit) 


\section{Discussion}

In the recent years, the desire for automatic image analysis tools or algorithms has been increasing and has so far been only partially satisfied. More and more labs thus create their own applets or plugins using commercially available programming languages like Matlab or with free software such as ImageJ. To use or to create these tools however often requires a well founded background in programming skills and mostly the features are bound only to very specific questions, as they are not conceived to serve any other purpose. In contrast to these programs, our novel image analysis tool, XtraCOunt, provides a widely accessible, free and easy to use platform that functions to semi-automate and standardize the analysis of light- or electron micrograph studies. No programming skills, special computer setups or software are needed to run our software and it can be applied to virtually any in-vivo model system such as mouse,flies, worms or yeast, and even in-vitro systems such as cell-free assays. It reduces the human bias and accelerates the speed of analysis immensely and thus enables the scientist to spend more of their valuable time for the interpretation of their, now more robust, data. In internal benchmark tests we have seen that the time spend for the analysis with the help of the program was decreased by up to $90 \%$. XtraCOunt should therefore provide the basis for more comparable studies and is intended to standardize, enhance and accelerate image analysis studies in a wide range of scientific fields.

As a novel feature to facilitate comparison of the morphologically diverse synapses in a standardized fashion, we integrated a rectangular binning feature that can be used in addition to the standard circular method. We propose the use of this feature in order to account for the irregular shape of C. elegans nerve terminals, as this grid is intrinsically normalized and serves thus as a better base for comparisons.

In the past, the main focus of vesicle distribution studies has been the gradient of vesicles through the entire nerve terminal from the presynaptic density. With increasing understanding of synaptic function, scientists have found the need to shift this focus to the active zone of synaptic terminal. Since the term of active zone could only be loosely defined in the past we have included a function in XtraCOunt that is able to generate reports solely summarising the regions close to the active zone and presynaptic density by omitting the rest. We term this combined region 'Periactive Zone'. The vesicle population closely associated with membranes only represents a fraction of vesicles in every terminal. However, what specific role these 
vesicles play and whether they represent the 'readily releasable' vesicle pool has been investigated but still remains to be determined in detail and in these future studies, XtraCOunt can play an important role .

Current limitation of the program will remain to be under future development to drive improvements where needed. So far only Tagged-Image-Files and Bitmaps can be analysed with XtraCOunt. Since these files are a given standard and most cameras and imaging software tools are supporting these file types we will certainly work to support more file types. Possibilities to include files spanning more or less that 1024 pixels in each dimension are currently at the debate. Since smaller files get to blurry and bigger files can not be displayed on most of the currrently available computer screens we nevertheless find that this resolution is a sufficient compromise for the current state of technical possibilities. To include a zoom option could help with the resolution problem but is in terms of developmental costs and complexity currently too far out of scope. However any desired changes and add-ons that would be broadening the scope of XtraCOunt will be considered for realisation as it is intended to facilitate trafficking studies for all scientists. 


\section{Chapter V. The cuticle of $d p y-1$ mutant nematodes}

\section{Introduction}

The cuticle of nematodes is a complex structure of highly cross-linked proteins and is mainly composed of collagens and cuticulins. In our studies we investigate the Caenorhabditis elegans dpy-1 gene and its orthologous gene from the satellite model system Pristionchus pacificus. DPY-1 proteins have a signal sequence, a von Willebrand Factor A (vWFA), a Zona Pellucida (ZP), a transmembrane domain and several regions of conservation.

A


B
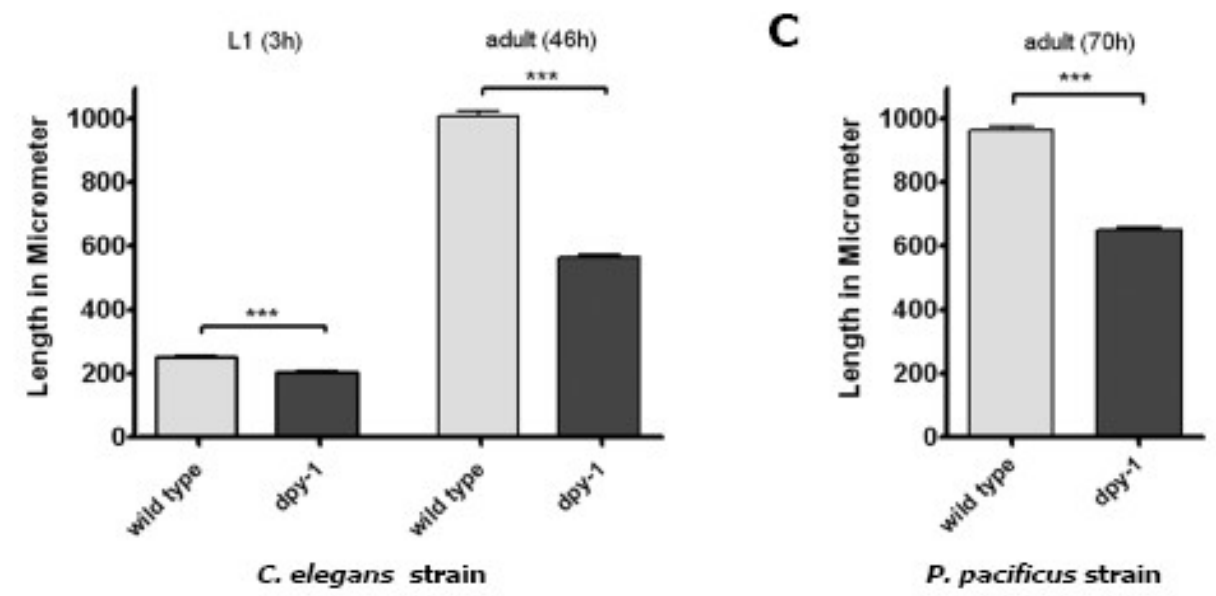

P. pacificus strain

Figure 31: The growth defect in dumpy worms

A) Bright field images of: (a) N2, (b) dpy-1 young adults (46 hrs), (c) wild type P. pacificus and (d) Ppa dpy-1. B and C) Lengths of staged animals at the L1 $(3 \mathrm{hrs})$ and young adult stage $(46 / 70 \mathrm{hrs})$. dpy-1 animals are significantly shorter than normal at all developmental stages. This is most obvious in adults but can be seen even in L1s. As the diameter of dpy-1 animals is close to normal (data not shown) they appear fatter than normal. 
Upon disruption of the gene the nematodes show movement problems and difficulties in maintaining a fully elongated form (Figure 31). Mutations in the C. elegans and P. pacificus dpy-1 genes both cause a similar strong dumpy phenotype. The measurements were carried out using the irregular distance measurement feature of XtraCOunt.

\section{dpy-1 expression correlate with molting events}

Expression analysis revealed a strong rise in DPY-1 production before the molting events of the worm (Figure 32), when it synthesizes a new cuticle and sheds the old one.

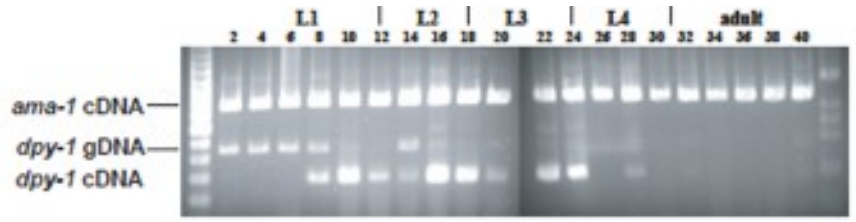

Figure 32: Expression of dpy-1 before molting events

The expression of $d p y-1$ transcripts from RNA from staged animals every two hours at $25^{\circ} \mathrm{C}$. Semi-quantitative PCR was used to detect products specific for $d p y-1$ cDNA (ama-1 control). Products amplified from trace genomic DNA are noted with gDNA. A peak before the indicated the molting events is clearly visible. (courtesy of B. Lakowski)

Although $C$. elegans can synthesize seven distinct cuticles and the cuticle of each stage has differences, the general structure of each cuticle is similar (Page and Johnstone 2007). The cuticle is synthesized by both the hypodermal(HYP) and the seam cells which lie just under the cuticle. The cortical layer over the seam cells has large longitudinal ridges, known as alae, and over the Hyp cells this layer is divided into narrow radial bands known as annuli and their dividing radial valleys, the annular furrows (Figure 33).

To examine further the effect of $d p y-1$ mutations on the structure of the cuticle, we used two established cuticular marker systems, a COL-19::GFP construct and a DPY-7 antibody. In the wild-type col-19::GFP is expressed during the late L4 stage and is incorporated into the

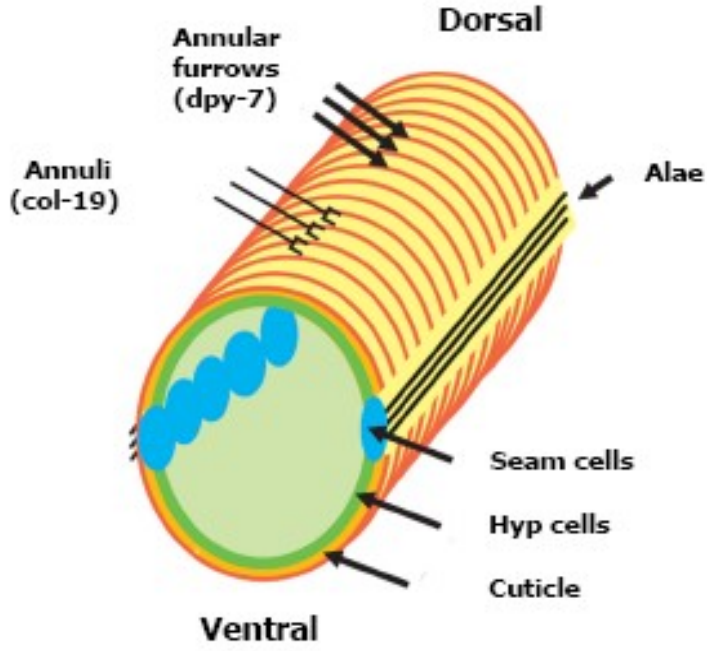

Figure 33: Schematic of the $C$. elegans cuticle

A section of the adult C. elegans body showing the annuli in yellow, the annular furrows in red, alae in black, seam cells in light blue and Hyp cells in green. (courtesy of B. Lakowski) 
adult cuticle in radial stripes along the dorsal and ventral sides of the worm that correspond to the annuli as well as in the longitudinal alae (Thein et al. 2003). Using a DPY-7 antibody the cuticle of wild -type N2 animals is stained in narrow stripes that underlie the annular furrows and the alae (McMahon et al. 2003).

\section{dpy-1 mutant nematodes display cuticle formation defects}

In $d p y-1$ mutants, the pattern of COL-19::GFP is very diffuse. col-19::GFP is expressed across both the dorsal and ventral surfaces of the worm (Figure 34). The COL-19::GFP does not appear in a regular pattern of parallel stripes divided by gaps as in wild type worms. Furthermore, using a DPY-7 antibody, the cuticle of N2 animals is stained in narrow stripes

wild type
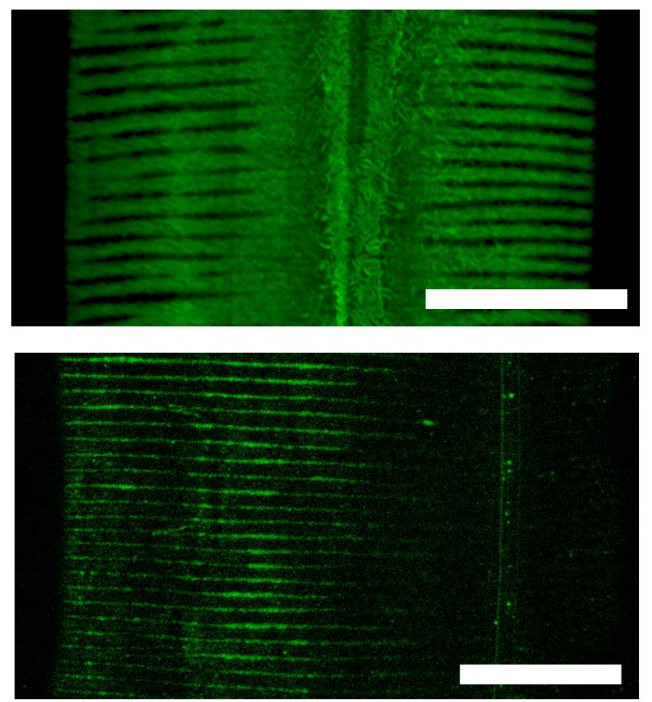

dpy-1
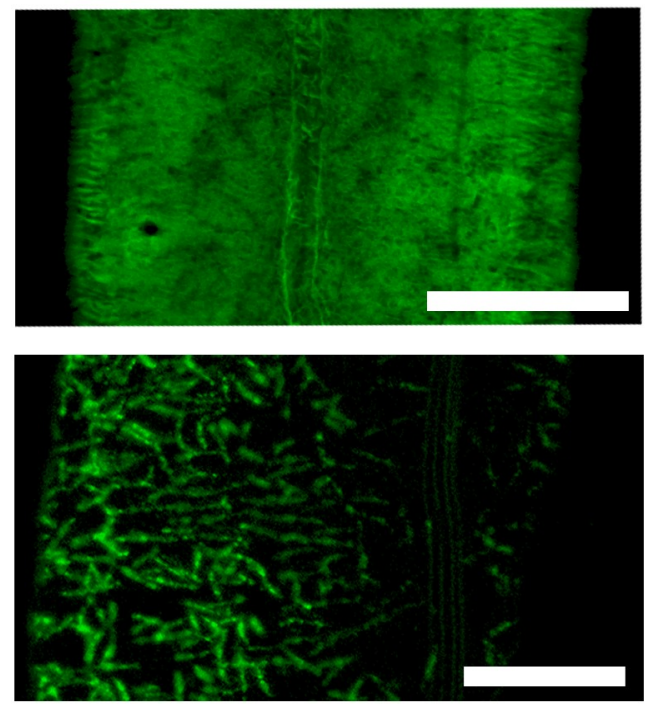

Figure 34: Annular patterning of $d p y-1$ mutants

In the wild-type adult col-19::g/p is expressed in radial stripes along the dorsal and ventral sides of the worm and correspond to the annuli of the worm, as well as in longitudinal bands over the seam cells. In $d p y-1$ mutants the pattern of COL-19::GFP is much less regular on the dorsal and ventral sides. COL-19::GFP is threadlike but col19::GFP is expressed all over the surface of the worm. The col-19::GFP is expressed more strongly in some regions than others but does not give a regular pattern of parallel stripes divided gaps. Using a DPY-7 antibody the cuticle of N2 adult animals is stained in narrow stripes underlying the annular furrows and the alae. In $d p y-1$ adults the DPY-7 staining is completely disorganized over the dorsal and ventral sides of the body. DPY-7 is present in short threads that are not oriented and show no clear pattern here. Most of the time, the pattern of DPY-7 under the alae is normal. Scale bar $=10 \mu \mathrm{m}$.

underlying the annular furrows and the alae. In $d p y$-1 adults the DPY-7 staining is completely disorganized in dorsal and ventral sides of the animals. DPY-7 is present, but only in short threads that show no clear orientation preference or pattern (Figure 34). 
This effect is furthermore seen on the surface of the cuticle, as it can be visualized by performing Scanning Electron Microscopy (SEM) with critically point dried nematodes. In this method, the water of the tissue (respectively all for the water substituted acetone), which needs to be extracted for the work in high vacuum, is evaporated in a single step under special temperature and pressure conditions (reviewed in Nail et al., 2004). As all solvent evaporates, surface tension issues from drying are circumvented and the fine tissue structure remains intact. The specimen can then be visualized by SEM. SEM allows to visualize the surface of a given sample in contrast to TEM approaches where primarily the interior content is visualized. The cuticles surface in dpy mutant worm clearly resembles the visualization by fluorescence microscopy (Figure 35).
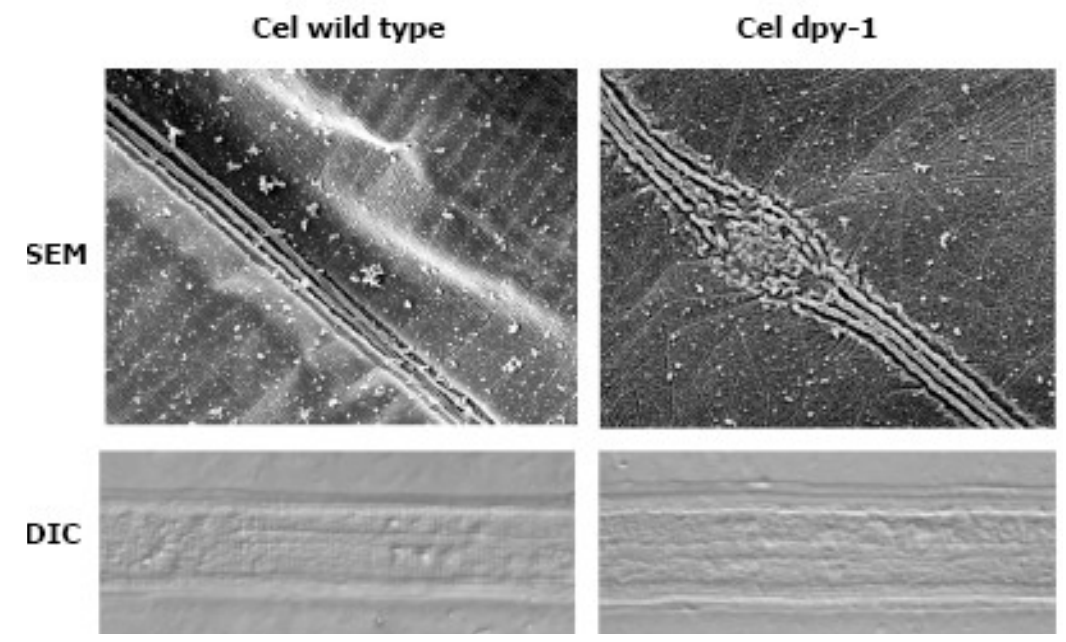

Figure 35: The cuticular defects of $C$. elegans $d p y-1$

Scanning Electron Microscopy (SEM) images of N2 and dpy-1(e1) adults. Bands of alae run through the centers of each image. The regular pattern of annular furrows is clearly evident in the N2. In the dpy-1 cuticle, short segments of annular furrows are connected in a fairly random pattern to generate a cuticle with a mosaic pattern of furrows. The dpy-1 cuticle in this image also shows a region of disrupted alae. DIC images of shed dauer cuticles from N2, and dpy-1 animals. Dauer alae are clearly seen at the top and bottom of each cuticle. N2 cuticles also show many narrow well spaced bands running perpendicular to the axis of the alae with their thickness suggesting that they are annular furrows. These bands are either completely absent, or not organized in to a regular equally spaced array, in dpy-1 cuticles. (courtesy of J. Hegermann - SEM and B. Lakowski - DIC)

Annular rings are present in wild type worms but are interconnected in the mutant nematodes. This effect can also be visualized on light microscopic level when investigating previously shed cuticles with differential interference microscopy (DIC) which clearly depicts a regular radial patterning that is absent in mutant worms. As a second, less penetrant effect, the misspatterning of the alae structure can be seen in about five percent of the $d p y$ - 1 mutant worms. Here the destruction of the regularly three-striped alae was also captured in the SEM 
experiment. As this effect was seen in C. elegans, which has only one alae, the appearance of P. pacificus cuticles, carrying multiple alaes, was similarly examined.

In P.pacificus the cuticle has an apparently more complicated surface structure than the C. elegans cuticle (Figure 36). The cuticle of wild-type P.pacificus has many prominent longitudinal ridges are running along the long axis of the worm which are often visible under high magnification light microscopy. These ridges are usually very straight and run parallel to each other with some small local regions of discontinuity where the regular lines converge or diverge due to changing diameter of the worm in different regions of the body.

In Ppa-dpy-1 animals this pattern is largely intact, however, in every animal examined there are some local discontinuities and mispatterning of the longitudinal bands. The cuticle of P. pacificus also shows radial bands that intersect the longitudinal bands at right angles. These bands presumably correspond to the $P$. pacificus annuli. The radial bands are divided by radial valleys, that most likly correspond to the annular furrows of the P. pacificus cuticle. As in Celdpy-1 mutants, Ppa-dpy-1 mutants do not have radial bands, or these bands are not organized into a regular pattern (best visible on DIC images). As this mispatterning is structurally very obvious we were able to deduce that this defect causes the dpy-penotype of worms in both species. The progressive nature of the phenotype is however not yet explained.
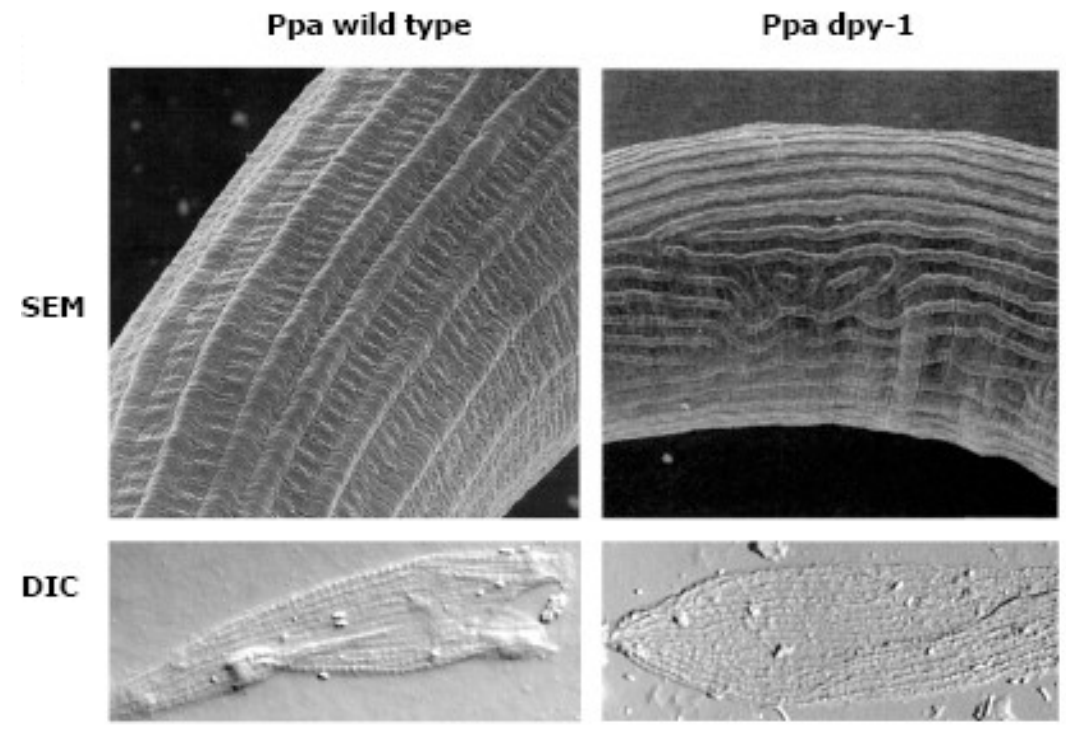

Figure 36: The cuticular defects of $P$. pacificus $d p y-1$

$P$. pacificus phenocopies the cuticular defects of C. elegans dpy-1 mutants very accurately for alae and annular furrows. (courtesy of R. Sommer -SEM and B. Lakowski -DIC) 


\section{Cuticle formation is progressively defective in dpy-1 mutant nematodes}

Interestingly, in $d p y-1$ mutant embryos, the pattern of DPY-7 is highly regular and looks very similar to wild type, an effect which can be seen on TEM level as well (data not shown). In contrast to the intact embryo cuticle the L2 and L3 larvae display a partially disrupted pattern of DPY-7 bands, while adult cuticles appear completely disorganized (Figure 37).

This indicates that in $d p y-1$ mutant animals the cuticle is initially patterned normally, but over

\section{dpy-1}

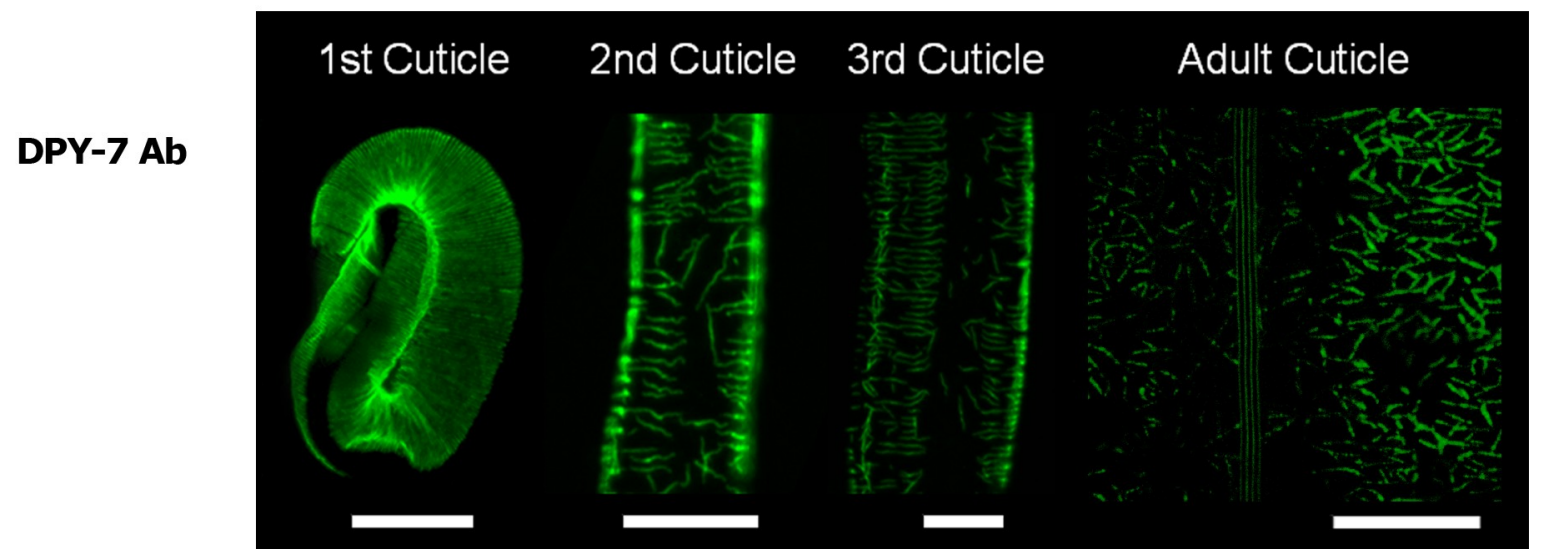

Figure 37: Annular misspatterning progress after molting

Immunofluorescence images of DPY-7 staining of $d p y-1(e 1)$ animals at different stages of development: late embryogenesis, L2, L3 and adult cuticles are shown. DPY-7 staining is largely normal in late embryogenesis but obvious defects start to appear in early larval stages and become progressively worse, leading to a highly disorganized pattern in adults. Scale bar $=10 \mu \mathrm{m}$.

time this pattern breaks down or is poorly replicated at each larval molt. Since each cuticle is synthesized in accordance to the patterning of the old cuticle, errors can accumulate over time. This explains the apparent progressive nature of the dumpy phenotype that is more obvious in adults than in L1s (compare adult to L1 in Figure 31).

\section{Discussion}

With our study, we could show that in the absence of DPY-1, animals display defects in the maintenance of the annuli and annular furrows in the cuticle of the nematodes species $C$. elegans as well as P.pacificus. In this investigation XtraCOunt measurements helped to accurately quantified worm body length in C. elegans as well as P.pacificus, and to measure regularity of the repeating structures within the cuticle. With its help were able the show for the first time the 
progressive nature of this dumpy phenotype and moreover link it structurally to the cumulative misspatterning of the radial running annuli of the worm by fluorescent microscopy approaches.

From the functional point of view the annuli most likely help to radially constrict the body and help to give the cuticle its flexibility by segmenting it. Once the rings of fibers that run below the annuli are not connected properly the missfunctional radial constriction of the worms interior content leads to the dumpy phenotype in shape. The worms are fatter and as they do not change their volume shorter. This combination leads to the perception of the worms appearing dumpy. Having this inflexible cuticle the movement by sinosoidal locomotion can no longer be maintained and their reduced traveling speeds can thus also be explained. As the worms seem not to be able to move, they just starve on the plates while the embryos inside of the worms continue to grow. Death of the worms most likely happens either by exploding from the inside, through the accumulated progeny and the weakened cuticle or via starvation as mobility becomes an issue at late adult stages.

By deciphering these functional characteristics of $d p y-1$ in the role of cuticle formation the description of the first ever documented phenotype (Brenner, 1974) in C. elegans is completed however the exact biomolecular mechanism will have to be of interest in future studies. A helping hint might be the fact that the $d p y-1$ structure resembles the one of cut- 6 , a cuticulin, active responsible in formation of the dauer cuticle. The life cycle of worm in low food environments in L1 stage can change to a persistent dauer-state resembling hibernation. Once food is sensed the life cycle is continued in the adult stage, skipping the normal L2 and L3 stages. A possible rescue of $d \not p y-1$ mutant worms by driving cut- 6 under a $d p y$ - 1 promotor might reveal their individual roles in the two possible life cycles of the worm. However, as the shed dauer cuticles already show the misspattering, this experiment will most likely, only milden the present phenotypic aberrations. Such an experimental result would, on the other hand, explain the presence of two such similar genes in one organism. 
Chapter V. The cuticle of dpy-1 mutant nematodes 


\section{Chapter VI: X-ray imaging of HPF TEM samples}

\section{Introduction}

As a correlative imaging attempt we have performed x-ray imaging on high-pressure frozen worms. In these experiment we tried to exploit the fact that in contrast to classical studies using electron microscopy, the radiation damage, the resulting dose limitation and limitations of the sample size are of less concern.

This part of the thesis work aims at non-invasive, radiation-damage-free $\mathrm{x}$-ray imaging of invivo-like preserved, multicellular organisms. For this purpose we used the well established sample-preservation and -preparation techniques of the EM-field. The following chapter evaluates the potential of x-ray imaging techniques and validates the general applicability of the preserving EM protocols in these techniques.

In short, holographic phase-constrast imaging and $\mathrm{x}$-ray fluorescence experiments were performed. These investigations were carried out using thin-sliced, epon-embedded nematodes as commonly used in EM studies. The results show that the analysis of the thin slices were at the threshold of reasonable signal-to-noise levels, at least for the current experimental parameters. Contrarily, 3D tomographic imaging of nematodes turned out to be an elegant method for non-invasive recording of whole-worm-tomograms at subcellular resolution.

\section{Strategies of phase contrast $x$-ray imaging}

In order to visualize the thin epon-sections, commonly used in EM studies, one has to consider the nature of the imaged sample. The interaction of hard $\mathrm{x}$-rays with matter (especially biological tissues) is very weak at high energy levels (Energy $=17.5 \mathrm{keV}$ ). Additionally the sample slices are very thin $(d=500 \mathrm{~nm}$ - which is still large when compared to EM studies) and thus the absorption of $\mathrm{x}$-rays within these specimen is very low. To model the imaging process the exiting wavefield $\Psi_{\mathrm{E}}$ after the sample is written as: 


$$
\Psi_{E}(x, y, z)=\Psi_{0}(x, y, 0) \cdot \mathrm{T}(x, y, \Delta z)
$$

where $\Psi_{0}(\mathrm{x}, \mathrm{y}, 0)$ describes the illumination-function and $\mathrm{T}(\mathrm{x}, \mathrm{y}, \mathrm{z})$ the object-transfer-function. The complex transmission function is (Paganin, 2006):

$$
\mathrm{T}(x, y, d)=e^{-i k \delta d-2 k \beta d},
$$

and where $k=2 \pi / \lambda$ is the wavenumber. The object transfer function consist of a refraction and an absorption component, which - in combination - are defined to be the complex refractive index $\tilde{n}=1-\delta+i \beta$ of a given material (Henke, Gullikson and Davis, 1993). The real part $1-\delta$ represents effects of refraction and the imaginary $\beta$ accounts for the effects of absorption. $\delta$ and $\beta$ are wavelength dependent and are typically very small for hard $\mathrm{x}$-rays.

In the case of the epoxy resin sections in our setup the absorption $\mathrm{A}$ is:

$$
A_{\text {epon-slice }}=1-\mathrm{e}^{-2(k \cdot \beta \cdot d)} \approx 9 \cdot 10^{-6}
$$

with density $\varrho_{\text {epon }}=1.2 \mathrm{~g} / \mathrm{cm}^{3}, \beta_{\text {epon }}=3.82 * 10^{-10}$ according to tabulated values (Henke, Gullikson and Davis, 1993; http://www.cxro.lbl.gov/) for an energy of $17.5 \mathrm{keV}$ (wavelength $\lambda \approx 0.7 \AA$ ).

This absorption is very low and additional biological content that could be considered, such as lipids or proteins, does not alter this fact significantly. The standard imaging procedures known from light- and transmission electron microscopy however require a significant absorption contrast in the image ${ }^{5}$. Additionally, under closer examination the absorptive component of the refractive index decreases much faster for higher energies than the refractive component ${ }^{6}$ and thus it became clear, that the refractive component is very worth to exploit.

After the technical development of third-generation synchrotrons seminal phase contrast x-ray imaging was performed (Snigirev et al., 1995; Cloetens et al., 1996). These imaging strategies use wave propagation to reveal the phase information and do not contain any optical elements in the path of the free-propagating wave (Pogany, Gao, Wilkins, 1997; Zabler et al., 2005).

In the case of the thin sample-slices $\left(\delta_{\text {epon }}=8.75^{*} 10^{-7}\right)$ the induced phase shift $(\Phi)$ is:

$$
\phi_{\text {epon }}=k \cdot \delta_{\text {epon }} \cdot d \approx 3.9 \cdot 10^{-2} \mathrm{rad} .
$$

As the worm is not a free-standing object - it is surrounded by the epon - one now has to consider the phase shift differences of the embedding media to the actual embedded material.

5 As an exception, in single particle EM studies the phase contrast is very important.

6 True for high energies and apart from absorption edges. 
As the specimen thickness is very low, the requirements of the projection approximation are fulfilled. For the case of cellular membranes idealized phase shifts are thus considered to be accumulating ( $\varrho_{\text {membrane }}=1.2 \mathrm{~g} / \mathrm{cm}^{3}$ ) over the entire section thickness. The phase shifts resulting from membranes $\left(\delta_{\text {membrane }}=9.06^{*} 10^{-7}\right)$ in the epon sections is therefore approximated to be:

$$
\phi_{\text {membrane }}=k \cdot \delta_{\text {membrane }} \cdot d \approx 4.0 \cdot 10^{-2} \mathrm{rad} \text {. }
$$

Biological organelles within the cells of the nematode, like protein dense mitochondria $(\varrho=1.4$ $\mathrm{g} / \mathrm{cm}^{3} ; \delta=1.01 * 10^{-6}$ ), would accordingly (ideally) shift the phase by:

$$
\phi_{\text {organelle }}=k \cdot \delta_{\text {organelle }} \cdot d \approx 4,5 \cdot 10^{-2} \mathrm{rad} .
$$

Therefore the estimated maximum difference in phase shift between the intracellular components and the embedding material $\left(\Phi_{\text {total }}=\Phi_{\text {epon- }}-\Phi_{\text {organelle }}\right)$ calculates to $6 \mathrm{mrad}$. Thus, although most tissue in the imaged epon-section will contain a mixture of all mentioned material, the biological samples within the thin epon-slices can be considered to be weak phase objects. Fortunately, these small magnitudes of phase shifts can be still converted into contrast changes in phase contrast imaging studies.

\section{$\mathrm{X}$-ray experiments allow propagation based phase contrast imaging}

The type of signal recorded from the diffracted and then propagated waves strongly depend on the propagation distance between the sample and the detector (propagation based imaging). The mathematical calculations to retrieve the desired original projections from the diffraction image heavily depend on the imaging regime and thus have to be chosen accordingly for each individual experimental setup.

In our case, the data was collected in the holographic regime. These holograms can be reconstructed directly, in a single step, using a back-propagation of the image from the detector to the sample plane - called in-line, holographic reconstruction or Gabor holography (Gabor, 1948). Reconstruction is achieved with following mathematical treatment ${ }^{7}$ of the detector image $I_{\text {detector: }}$ :

7 Note that for this type of reconstruction the paraxial approximation requirements have to be fulfilled, which basically means that all rays only make a small angle with respect to the optic axis (Paganin, 2006). Therfore, it can be shown that this divergent beam approach may be treated like a parallel beam setup when accounted for by the factors $Z_{\text {eff }}$ for the effective propagation distance and by the Magnification $\mathrm{M}$ given by $\mathrm{M}=1+\mathrm{z}_{2} / \mathrm{z}_{1}$ (Fuhse et al., 2006). 


$$
\Psi_{E}(x, y)=F^{-1}\left(F\left(I_{\text {detector }}(x, y)\right) \cdot e^{(-1) i k \cdot z_{e f} \cdot \kappa}\right)
$$

where $\mathrm{F}$ denotes the Fourier transform with $\mathrm{F}^{-1}$ being its inverse transformation. The exponential part is commonly termed the Fresnel-propagator where the effective propagation distance $z_{\text {eff }}$ is given by the distances between source and sample $z_{1}$ and sample and detector $z_{2}$ in the form of $z_{\text {eff }}=\left(z_{1} * z_{2}\right) /\left(z_{1}+z_{2}\right)$ (Fuhse, 2006) and $\kappa$ accounts for the dimension of the image in the Fourier-space with $\kappa=\sqrt{1-\left(k x^{2}+k y^{2}\right) / k^{2}}$ (Paganin, 2006) where kx and ky denote the spatial frequencies.

\section{Phase contrast imaging can be combined with tomography}

Phase contrast imaging can be combined with tomography, to which end an image series is recorded while the object is rotated. The recorded images can then be back-projected into 3Dvolume with the filtered-back-projection algorithm that is based on the concepts of the Radon transform (Radon, 1917) and the Fourier-slice theorem (Kak and Slaney, 1988).

In medical tomograms signals recorded for a pixel is only integrand of all absorption coefficients over the ray trajectory. For phase images of nano- or mircoscale objects the signal arises from interaction of neighboring rays and thus the signals are not independent from each other. The consequence is that a reconstruction of the images needs to be performed to account for the phase contrast nature of the images. This can be done before or during final reconstruction of the 3D-volume.

To be able to reconstruct the information from near field phase contrast images, as used in our tomography study, the modified-Bronnikov algorithm (Bronnikov, 2002; Groso, Abela and Stampanoni, 2006) was applied - an algorithm which is designed to work best for low absorption samples and that reconstructs the phase contrast images.

The major improvement of this form of reconstruction approach is that the direct recovery of the refractive index in 3D for pure phase objects from a single measurement becomes possible (Bronnikov, 1999, 2002). This is a major improvement to the general strategies commonly 
used, because it allows for reduced radiation damage - which becomes especially important for in-vivo imaging attempts. Since the phase retrieval is directly approached this method does not suffer from stagnation problems in the process of reconstructions like other methods in coherent diffractive imaging (Nugent, 2009).

\section{Strategies of fluorescence $x$-ray imaging}

In addition to a plain visualization of the sample we wanted to gain information about the chemical composition of the specimen. This can be done in a spectroscopic fashion with the help of x-ray fluorescence. As previously mentioned the absorption of x-rays in the specimen is characterized by the intensity loss of an x-ray ray beam passing through a sample.

The resulting intensity can be calculated by the Beer-Lambert law (Bouguer, 1727; Lambert, 1760; Beer, 1852; Als-Nielson and Mc Morrow, 2000):

$$
I=I_{0} e^{-\mu d},
$$

where $I_{0}$ is the starting intensity, $d$ is the sample thickness and $\mu$ is the energy dependent absorption coefficient.

The observed absorption is due to interaction of the x-rays with the atom shells in the sample in three distinct ways, namely the photoelectric effect where electrons are removed from the atomic shells, as well as the the elastic- (Thompson-) and inelastic (Compton) scattering of the photons. The absorption coefficient $\mu$ of a given material is thus given by:

$$
\mu=\mu_{\text {photo }}+\mu_{\text {thompson }}+\mu_{\text {compton }} \cdot 8,9
$$

\section{Photoelectric effect leads to characteristic fluorescence}

If photoabsorbtion is followed by ionization of an atom, the resulting inner shell hole is filled

8 For the thompson effect the photons of the incoming wave leave the sample with an unaltered wavelength, but are scattered away from the ray of projection. This process is termed extinction.

9 The effect of the pair production is neglected here, as the energy levels in this study are not high enough for this effect. 
by a transition of an outer shell electron. The energy difference is released through emission of a fluorescent photon or the Auger effect.

The energy difference is written as $\Delta E=h \cdot v$, where $\mathrm{h}$ is the Planck constant and $\mathrm{v}$ the frequency of the photon. The emitted radiation is thereby characteristic for any given element. This process is called $\mathrm{x}$-ray fluorescence. Moseleys law describes the emitted radiation with the help of the atomic number $Z$ of a given element as (Moseley, 1913):

$$
\Delta E=R(Z-\sigma)^{2}\left(\frac{1}{n_{1}^{2}}-\frac{1}{n_{2}^{2}}\right),
$$

where $\mathrm{R}$ is the Rydberg-constant, $\sigma$ is the shielding constant. $\mathrm{Z}-\sigma$ describes the effective atomic number $\left(\sigma=1\right.$ for $\left.\mathrm{K}_{\alpha}\right) \cdot \mathrm{n}_{1}$ and $\mathrm{n}_{2}$ are the main quantum numbers with $\mathrm{n}_{2}>\mathrm{n}_{1}$.

The lines of the spectrum are ordered to K-, L-, and M-series according to the filled shell and further differentiated by greek letter subscripts indicating the origin of the filling electron. For the identification of a given element the $\mathrm{K}_{\alpha}$ is in general sufficient, but all other lines like $\mathrm{L}_{\alpha}$ or $\mathrm{M}_{\alpha}$ may be used as well, especially if the energy of the incoming ray is to low to remove electrons from the K-shell. ${ }^{10}$

\section{The Auger-effect competes with the fluorescence}

The energy released by the conversion to the ground state may also be transmitted to other electrons of the atom. The atom is then ionized twofold. This radiation-free effect is termed Auger-effect. As a consequence of this effect the spectral lines of a series are not as prominent as to be expected by the number of free-electron positions. The quantification of this effect, the fluorescence yield, is given by the ratio of emitted photons and open electron positions. The yield is varying between the different electron shells of a atom and is generally proportional to the atomic number of the observed element.

The fluorescence-yield W can be approximated with help of the shell-specific constants A, B and C by (Burhop, 1955):

$$
\left(\frac{W}{1-W}\right)^{\frac{1}{4}}=A+B Z+C Z^{3} \text {, where } \mathrm{Z} \text { is the atomic number. }
$$

The Auger-effect is most prominent for elements with a low atomic number as their inner electrons are less strongly bound. For the xray-spectroscopic detection of elements with low

10 In case a given element is present in varying chemical bindings the lines might be shifted in the different bonding states. This phenomenon is exploited in NEXAFS studies. 
atomic number the fluorescence-yield is the limiting factor.

\section{Fluorescence of epon-embedded nematode sections was measured in this study}

To reveal the elemental composition within thin epon slices from TEM microscopy in a noninvasive fashion we have used $\mathrm{x}$-ray fluorescence. X-ray fluoresence images are recorded not as a full field but in scanning fashion. The matter within the focal spot is activated by high energetic photons and begins to emit characteristic fluorescence spectra based on its elemental composition (Hall, 1961). This information can then be recorded for the entire object simply by scanning all the way across it (usually performed by translation of the object rather than the source). By fitting the recorded spectra to the known elemental emittance peaks, the identification of the contribution for all detected elements is possible (Solé et al., 2007). ${ }^{11}$

11 To minimize the detection of scattered photons the experimental setups detect fluorescence in an angle which close almost perpendicular to the incident beam. In our case the sample plane was tilted $60^{\circ}$ with respect to the incoming ray and the fluorescence detector was positioned in an angle of $28^{\circ}$ to the specimen, giving a total ray tracing angle of $88^{\circ}$. The experimental setup shows a schematic representation of this in the following results section. 


\title{
Results from $x$-ray holography studies of TEM sections
}

Thin-sections of $500 \mathrm{~nm}$ thickness were imaged using the the inline-holography setup (Figure 38) of the ID22NI at the ESRF in Grenoble, France and subsequently analyzed by TEM. However, the electron microscopic visualization of $500 \mathrm{~nm}$ thick samples with $80 \mathrm{kV}$ electron beam heavily distorts and finally destroys the samples. Nevertheless, the TEM analysis has to be performed in order to evaluate the quality of the results of the reconstruction.

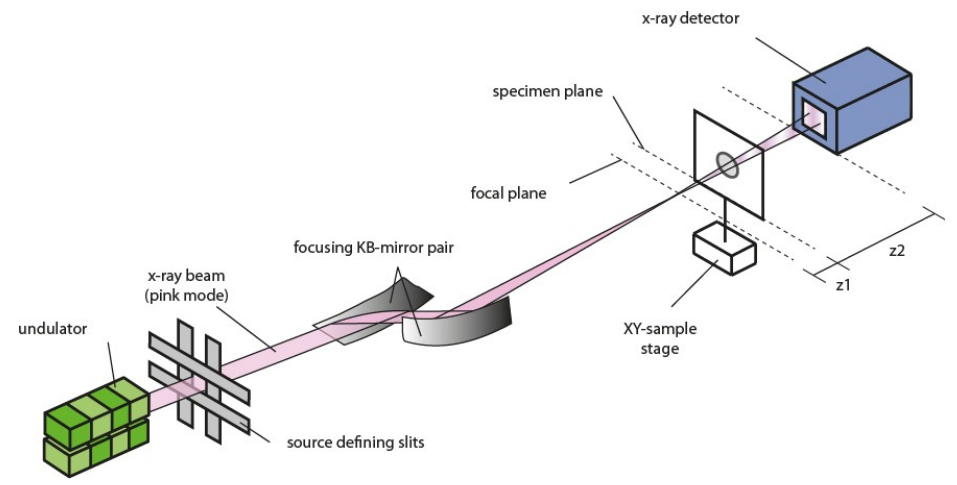

\author{
Experimental specifications: \\ Pink-mode x-ray beam $(17.5 \mathrm{keV})$ \\ KB-mirror focus $=130 \mathrm{~nm} * 140 \mathrm{~nm}$ \\ Specimen thickness $=500 \mathrm{~nm}$ \\ $10^{11}$ photons per second \\ $24 \mu \mathrm{m}$ LSO:Tb scintillator \\ FRELON 2000 CCD \\ $0.96 \mu \mathrm{m}$ pixel size \\ Exposure time $=5 \mathrm{~s}$. \\ $\mathrm{z} 1=25 \mathrm{~mm}, \mathrm{z} 2=526 \mathrm{~mm}$. \\ Magnification $=1+\mathrm{z} 2 / \mathrm{z} 1=21 \mathrm{x}$ \\ Effective pixel size $=45.6 \mathrm{~nm}$
}

Figure 38: ID22NI beamline - holography setup

The holographic setup at the ID22NI at the ESRF, France was tested for applicability in epon-slice imaging. The schematic layout of the endstation is depicted above. Experimental specifications are given on the right. The beamline experiment was performed by A. Beerlink, T. Salditt and coworkers.

The direct holographic reconstruction clearly depicts the image specimen (Figure 39) but the reconstruction quality is reduced by large artifacts (halos, lines and stripes). These artifacts are originating from several sources. At first the beam itself has intensity fluctuation within both the $\mathrm{x}$ - and the $\mathrm{y}$-axes originating from the used focusing mirrors. Furthermore the algorithm has problems dealing with samples spanning across the entire field of view - which the ultrathin section substantially exceeds. Secondly, reconstructions from in-line holograms suffer intrinsically from the degradation of the so-called twin-image. This problem cannot be solved with holographic reconstruction but requires more sophisticated treatment such as recently described strategies (Giewekemeyer et al., 2011). However, by convolution with a Gaussian blur function ( $\sigma=2$ pixel) noise can be smoothed out without losing too much image information, which finally enhances the quality of the generated results. 


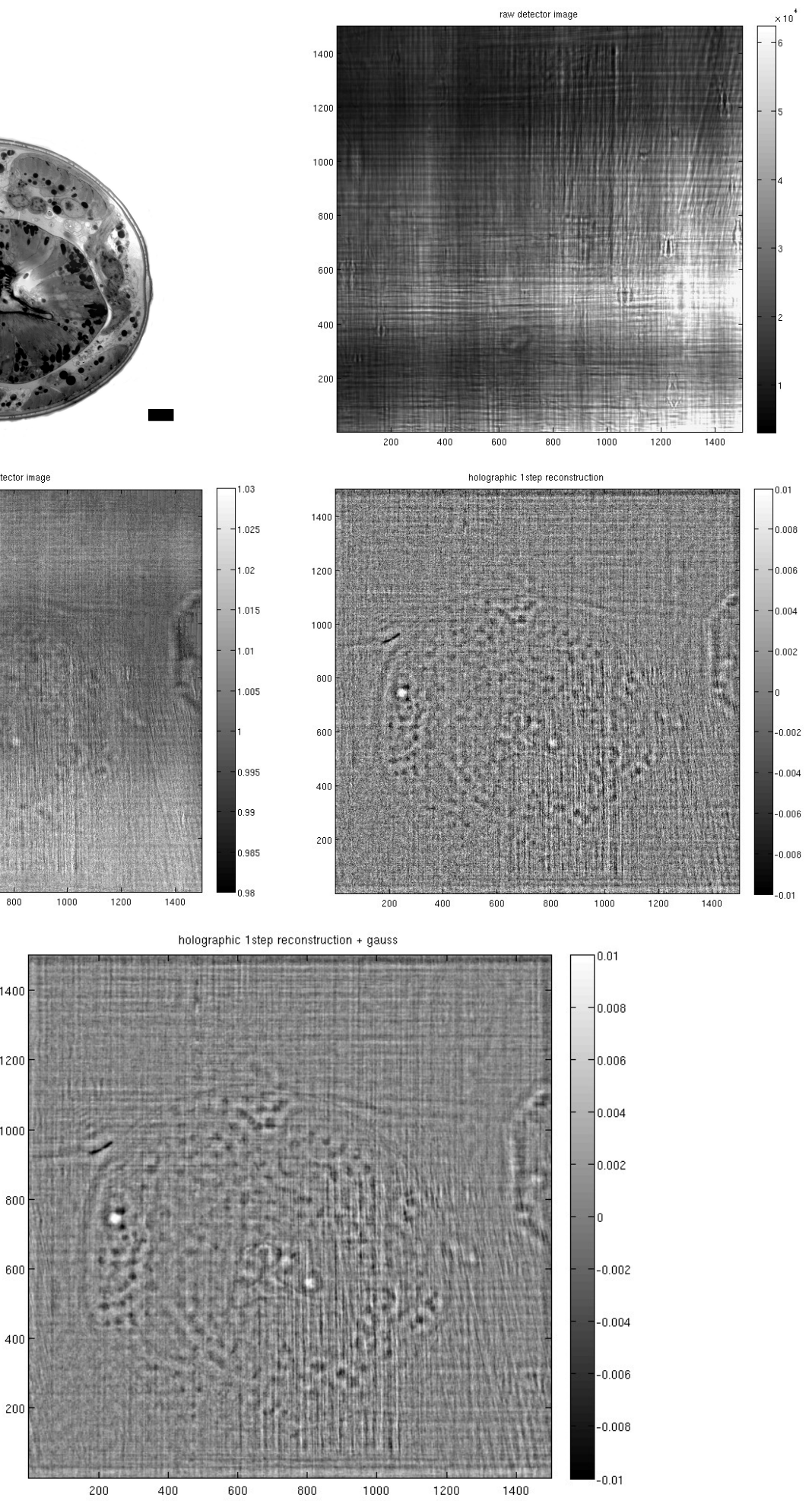

Figure 39: X-ray holography reconstruction of sectioned TEM samples

$500 \mathrm{~nm}$ thin sections are reconstructed from $\mathrm{x}$-ray holography experiments. The quality of resolved images is lowered by algorithmic and optical artifacts. Scale bar $=5 \mu \mathrm{m}, \mathrm{x}$-ray resolution $=130 \mathrm{~nm}$. Used Matlab routines were adapted from previous studies (Krüger, 2010; Giewekemeyer, 2011). 
An iterative approach that propagates the image back and forth between the sample and detector plane allows the use of constraints that allow to enhance the reconstruction. The original algorithm developed (initially without support constraints) by Gerchberg and Saxton (Gerchberg and Saxton, 1972) it is based on iterative projection and propagation using the Fresnel-propagator. The propagation from the detector back to the sample plane can be performed by the following equations.

$$
\Phi_{\text {sample }, n}(x, y)=F^{-1}\left(F\left(I_{\text {detector }, n}(x, y)\right) \cdot e^{-i k \cdot z_{\text {ef }} \cdot \kappa}\right),
$$

which accordingly propagates the image into the sample plane. In the sample plane the constrain, that no absorption takes place, can be used to normalize the image

$$
\Phi_{\text {sample } n^{\prime}}(x, y)=\frac{\Phi_{\text {sample }, n}(x, y)}{\left|\Phi_{\text {sample }, n}(x, y)\right|}
$$

This normalized image can then be propagated

$$
\Phi_{\text {detector }, n+1}(x, y)=F^{-1}\left(F\left(\Phi_{\text {sample }, n^{\prime}}(x, y)\right) \cdot e^{+i k \cdot z_{e f} \cdot \kappa}\right)
$$

to update the original detector image amplitude with the new, absorption normalized and corrected version of the detector image.

$$
\Phi_{\text {detector },(n+1)}=\sqrt{I_{\text {detector }, 0}(x, y)} \cdot \frac{\Phi_{\text {detector }, n+1}}{\left|\Phi_{\text {detector }, n+1}\right|}
$$

This improved result can now be fed into the first step algorithm instead of the first guess from the holographic reconstruction by:

$$
\Phi_{\text {sample },(n+1)}(x, y)=F^{-1}\left(F\left(\Phi_{\text {detector },(n+1)^{\prime}}\right) \cdot e^{-i k \cdot z_{e f f} \cdot \kappa}\right),
$$

This procedure $(\mathrm{i}$ - iv) can be performed iteratively as denoted by the subindex $\mathrm{n}$ where $\mathrm{n}=0$ represents the original recording. The iteration can be aborted once a well chosen abort criterion is reached. The appropriate choice for such a criterion is very critical, as too many iterations will lead to the (over)amplification of the non-desired, noisy image parts. Commonly the abort criterion evaluates the update step by measuring the error between the diffraction pattern from the reconstruction and the measured intensity. This method is optimal for low noise contributions but can however fail if noise dominates the measurement.

For our, relatively artifact prone, image a visual inspection was performed as an abort criterion. Best results are obtained with 10 iterations (Figure 40) and a smoothing Gaussian ( $\sigma=2 \mathrm{px}$ ). 


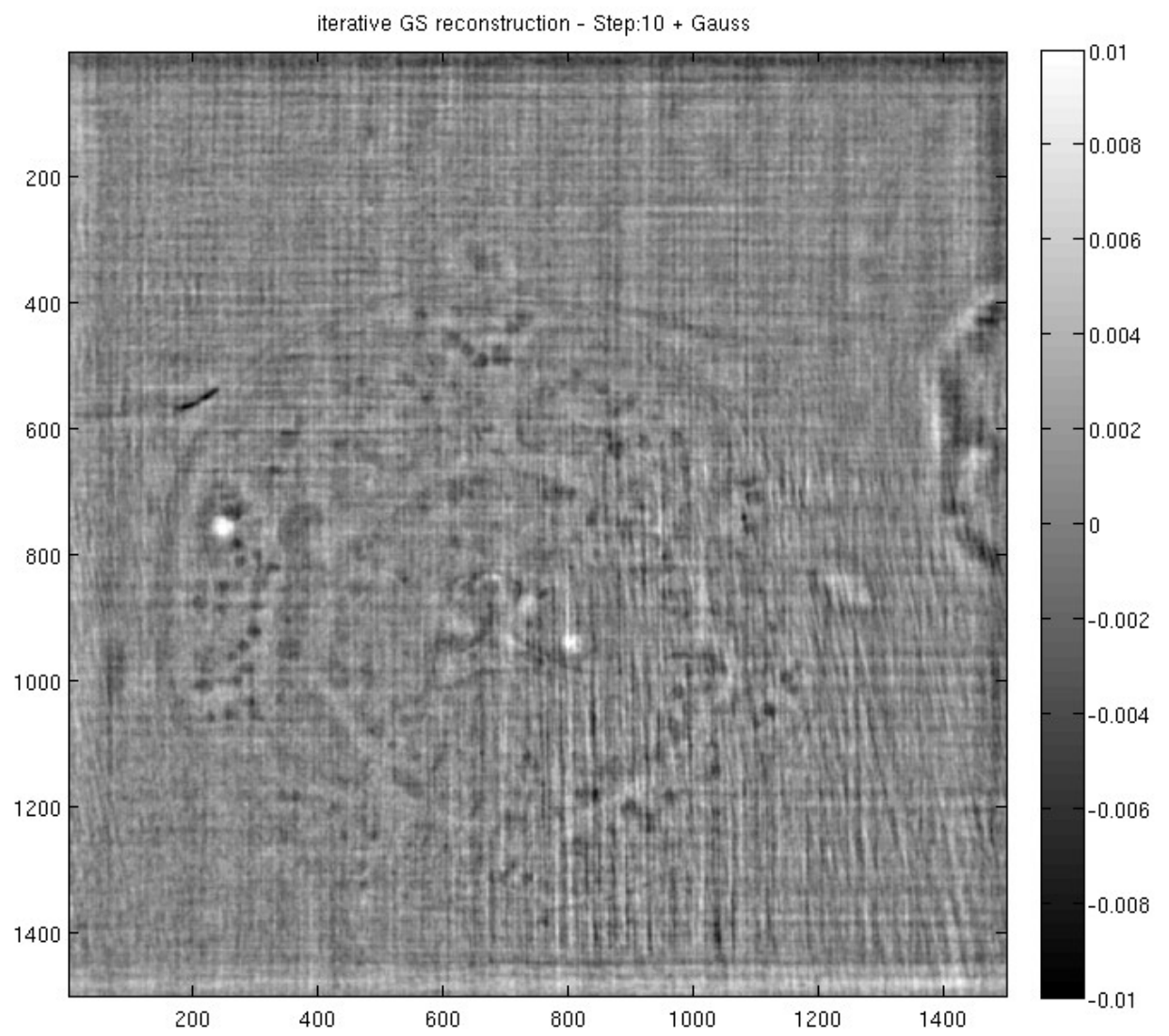

Figure 40: Iterative reconstructions of sectioned TEM samples

Ten Gerchberg-Saxton algorithm iterations with a subsequent Gaussian blurring show an improved reconstruction quality when compared to the direct holographic reconstruction. Field of view $=100 \mu \mathrm{m}$.

Like the holographic reconstruction the iterative reconstructed image was not artifact free, but despite of these errors, the tissue structure of the worm is discernible and subcellular structures such as mitochondria can be better distinguished. Most halos around the internal structures disappeared, which can be seen as an improvement compared to the holographic reconstruction. But to conclude, the method of holographic imaging of thin-sliced samples has proven to be able to visualize the biological, thin-sliced specimen. Additionally it may be noted that 1 . the bad quality of the reconstruction is may be due to shortcomings of the measurement (lack of coherence, sample drift, etc.) which hopefully can be overcome in the future and 2. a proof of principle for non-heavy-metal-stained tissues has to follow. 


\section{X-ray tomography enables Whole-Worm-Tomography}

For the three-dimensional reconstruction of large samples, electron tomography is limited by the large absorption through thick specimen. This issue which can be neglected if $\mathrm{x}$-rays are used instead because their interaction with the specimen is very limited. Therefore, the created beam damage is minimized and thus imaging can be performed by recording a far higher number of views as compared to electron microscopy. Furthermore, it is possible to fully rotate the specimen by $180^{\circ}$ to avoid the usual missing-wedge problem of EM sample holders.

In order to test the applicability of $\mathrm{x}$-ray imaging strategies and setups on HPF processed worms we imaged complete worms at the TOMCAT beamline at the SLS in Villigen, Switzerland (Figure 41) in their standard parallel beam setup.

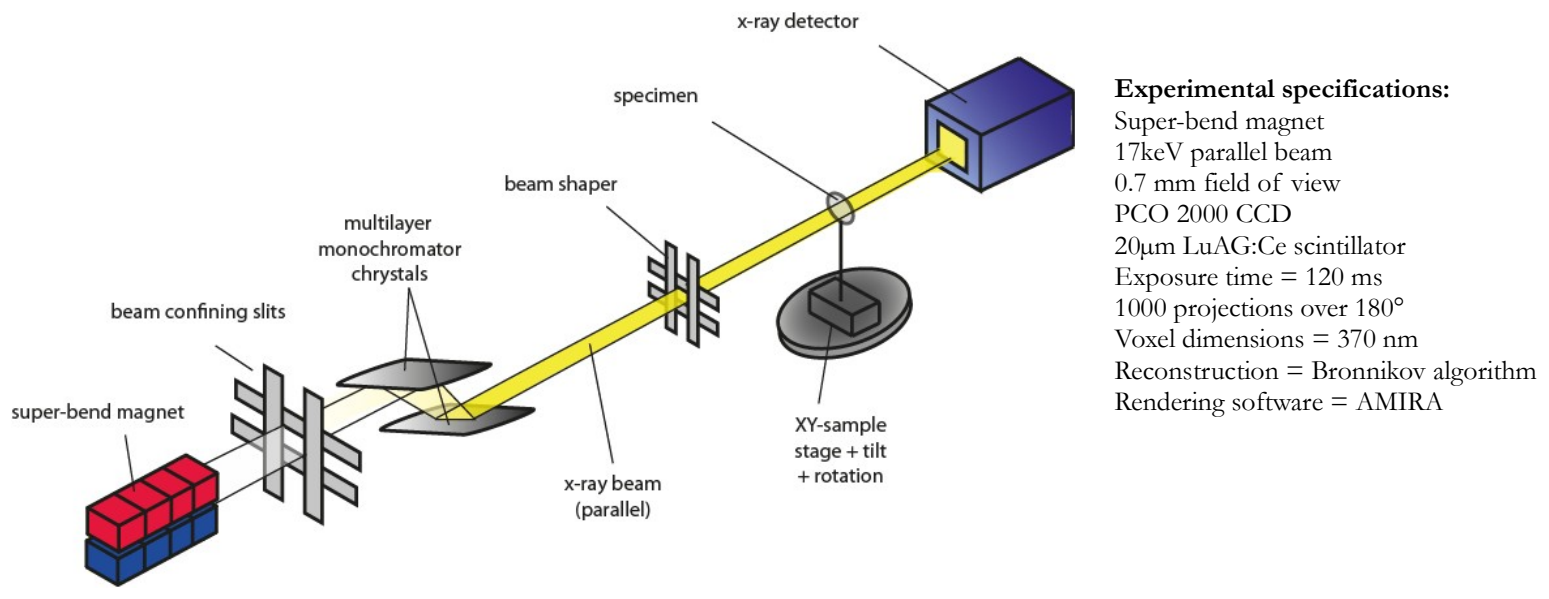

Figure 41: TOMCAT beamline - parallel beam tomography setup

The tomographic beamline setup at the SLS, Switzerland was tested for applicability in epon-block imaging. The schematic layout of the endstation is depicted above. Experimental specifications are given on the right.

The object of interest is an entire nematode embedded in an epoxy resin block. Objects of this size cannot be imaged in conventional electron microscopy and thus this test is more of an attempt to bridge the sample size gap between optical- and electron based microscopy. 

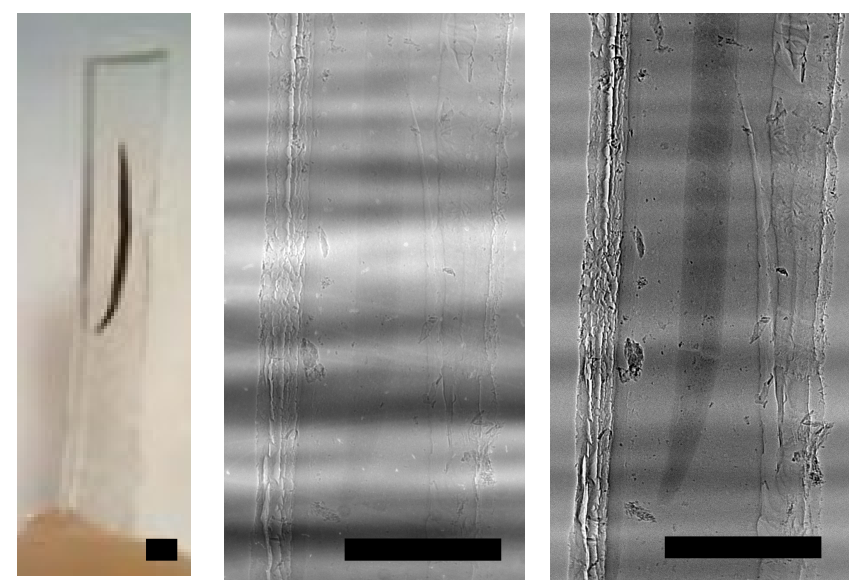

Figure 42: $2 \mathrm{D}$ projection images of a fully embedded worm

Left: A thin epon foil with an embedded worm was mounted onto a specimen holder for the rotational unit at the TOMCAT beamline at the SLS in Villigen, Switzerland. Middle: A raw projection image. Right: A representative flatfield-corrected projection for the tomography calculation is shown. Scale bar $=100 \mu \mathrm{m}$.

For this experiment the input energy was tuned to $17 \mathrm{keV}$ to reduce the absorption of this $100 \mu \mathrm{m}$ thick block down to about 20 percent, an absorption value where the application of the Bronnikov algorithm is starting to be feasible. For the optimal visualization of tomography
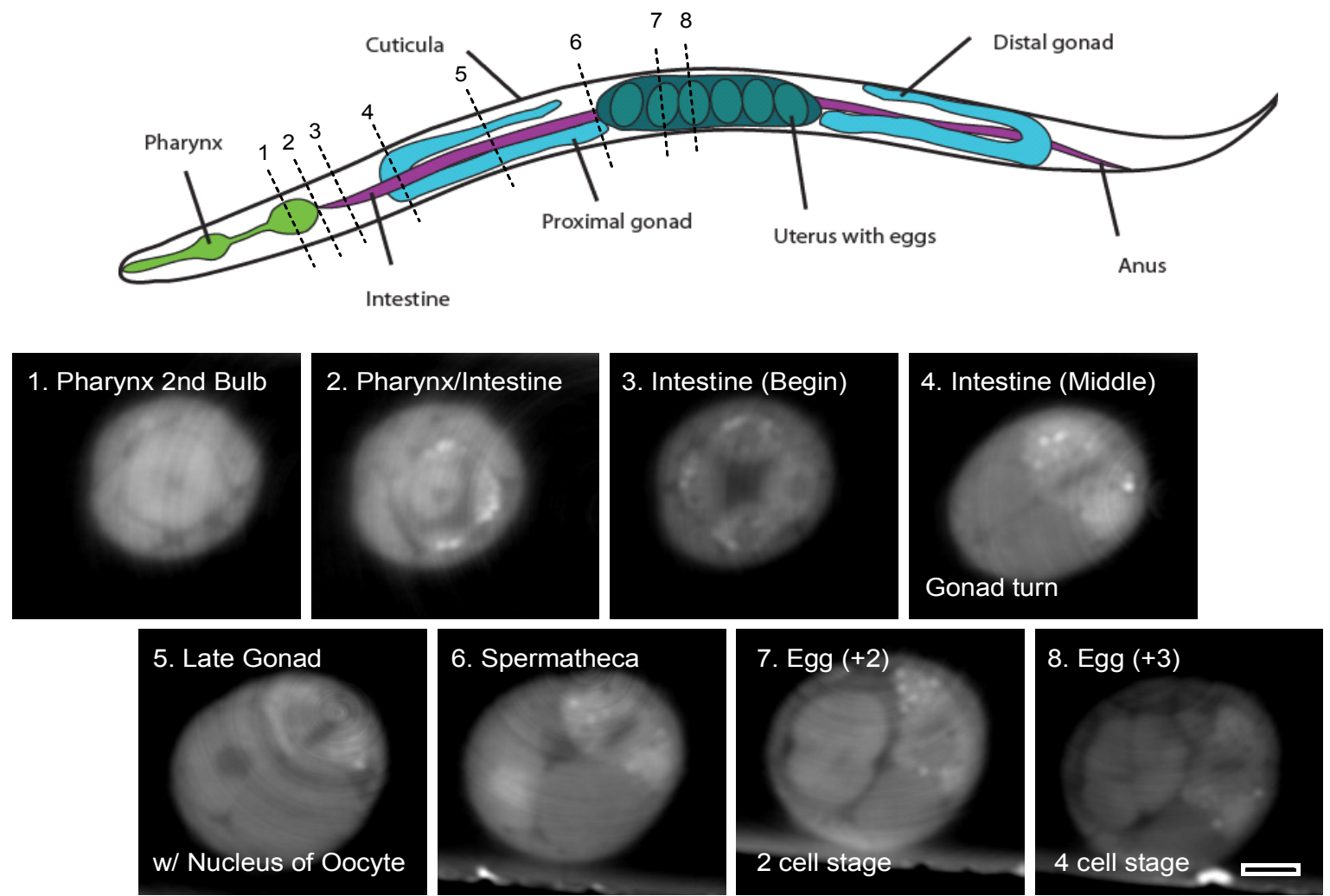

Figure 43: Virtual slices of tomographic reconstruction

A schematics indicates the position of eight virtual slices from the reconstruction with the modified Bronikov algorithm. The organ structure is clearly discernible in the slices and subcellular components such as mitochondria, nucleii or vesicular content can be identified easily. Isotropic voxel dimensions $=370 \mathrm{~nm}, 1800$ slices calculated, scale bar $=12 \mu \mathrm{m}$. 
results a rendering of the segmented data (in this case manually segmented) was performed with the software package of AMIRA (Figure 44).

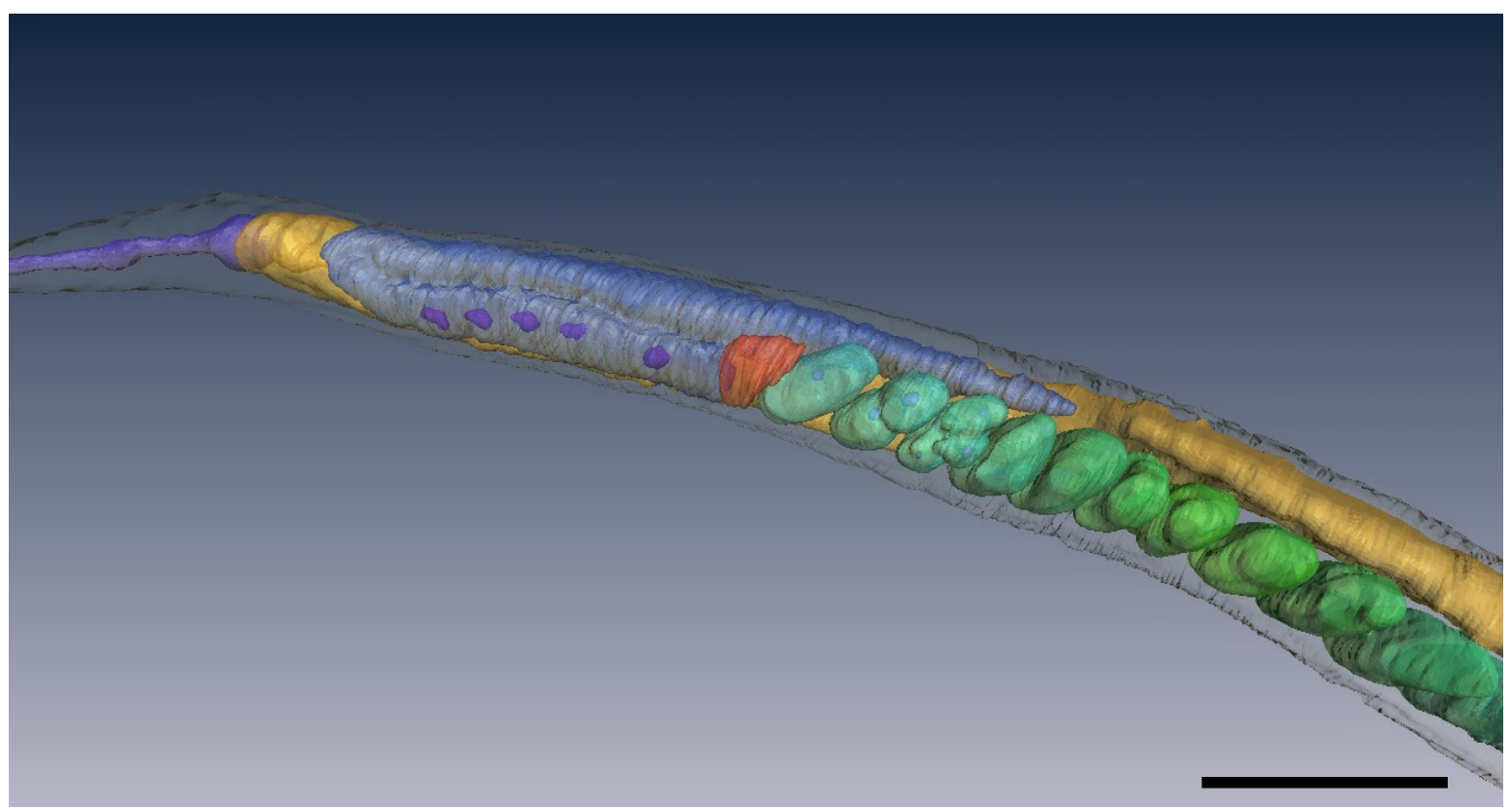

Figure 44: Modeling of reconstructed volume visualizes the nematodes tissue anatomy

A surface rendered model generated with the AMIRA software package. Cuticle is shown slightly transparent. Internal organs are indicated by the following colors: dark blue - pharynx, beige - intestine, blue - gonad, red spermathecae, cyan to green - maturing embryos. Isotropic voxel dimensions $=370 \mathrm{~nm}$. Scale bar $=100 \mu \mathrm{m}$.

Pharynx (dark blue) and intestine (beige) are easily distinguished in the worm (cuticle is marked transparent). One can appreciate the quality of the data best in the regions around the spermatheca. Once the unfertilisided oocytes (gonad - blue) pass through the spermatheca (red) they become fertilized and start to undergo cell division processes (cyan to green) in order to form the embryo. The model is clearly depicting the 1cell- , 2cell-, 4cell- and 8cellstage in the maturation process. The individual nuclei can be clearly separated in those eggs.

To conclude, this whole organism tomography approach was very successful, as it finally bridges the gap of sample size between light and electron microscopy. This experiment shows that whole-worm-tomography (WWT) can be realized with a resolution comparable to low level electron microscopy while at the same time working completely non-invasive. 


\section{$X$-ray fluorescence of sliced TEM samples}

The thin epon slices from EM studies were imaged by x-ray fluorescence (XRF) setup (Figure 45) of the ID21 beamline at the ESRF in Grenoble, France (Ducic et al., 2011).

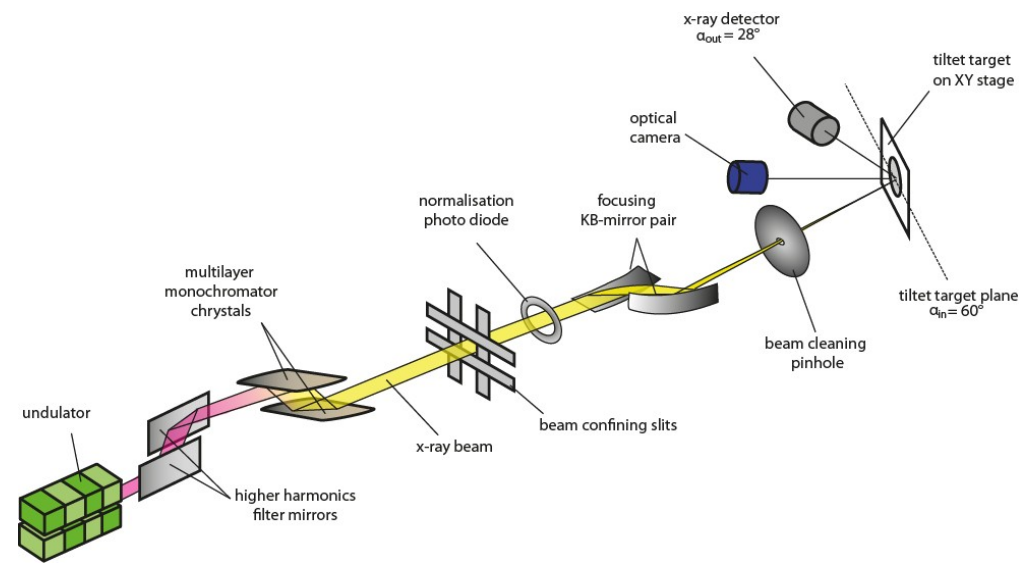

Experimental specifications:

Monochromatic x-ray beam

Recording energy $=$ up to $7,2 \mathrm{keV}$

$\mathrm{KB}$-mirror focus $=330 \times 800 \mathrm{~nm}$

Specimen thickness $=500 \mathrm{~nm}$

HPF freezing + osmium fixation

Energy dispersive Roentec detector

Energy resolution $=170 \mathrm{eV}$

Dwell time $=150 \mathrm{~ms}$

Step-size of $300 \mathrm{~nm}$.

Evaluation software $=$ PyMca

Figure 45: ID21 beamline - microfluorescence setup

The microfluorescence beamline ID21 at the ESRF, France was tested for applicability in epon-slice imaging. The schematic layout of the endstation is depicted above. Experimental specifications are given on the right. Experiments were conducted by A. Beerlink, T. Ducic, T.Salditt and coworkers.

The results from the XRF microscopy are fitted with PyMCA (Solé et al., 2007) to determine the contribution of desired elements to the recorded signal (Figure 46).

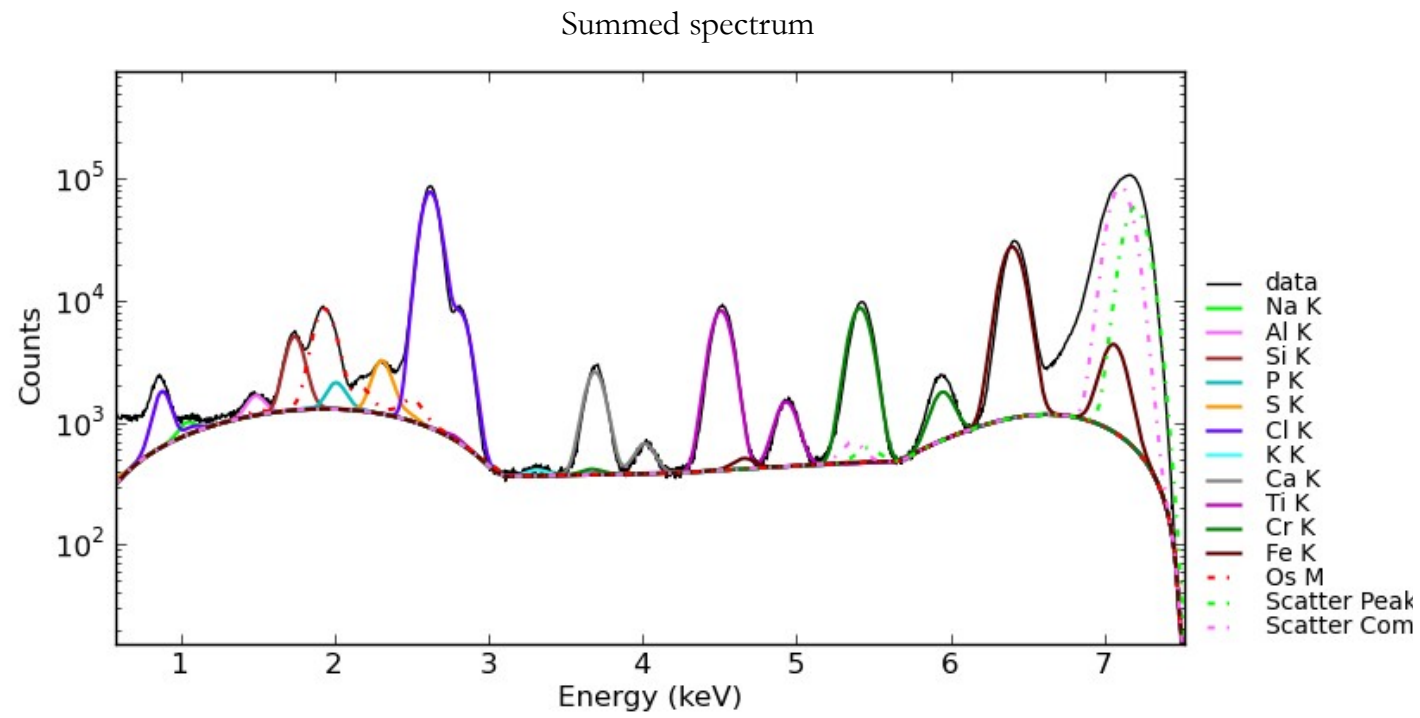

Figure 46: Energy spectrum of an ultrathin epon-slice including a nematode cross-section

With PyMCA, a tool to evaluate the recorded energy spectra (Sole et al., 2007), the specific contribution of elements to the signal can be approximated by fitting the recorded spectrum to known elemental emittance peaks. 
By focusing on specific energy ranges the elemental distribution between the different tissues in the worm can be visualized and subsequently compared to a TEM image of the slice (Fig. 47).
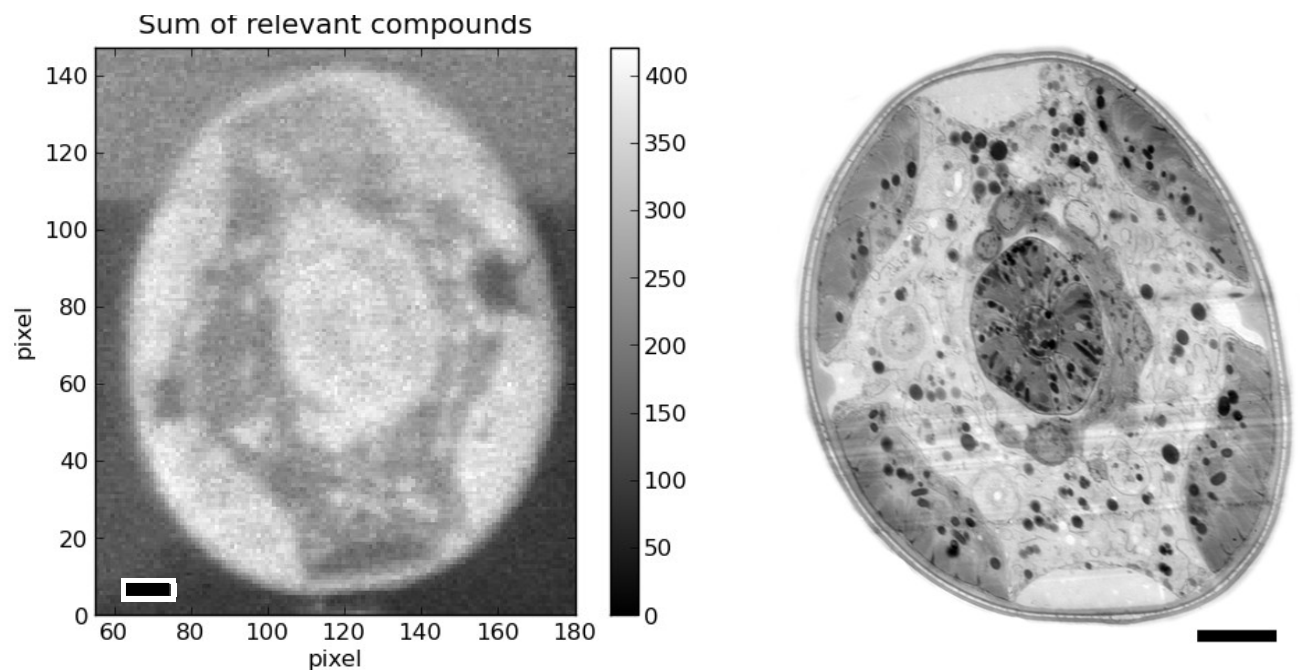

Figure 47: X-ray fluorescence of a ultrathin epon slice including a nematode cross-section

Elemental distributions of $500 \mathrm{~nm}$ thin sections of EPON embedded worms are visualized in $\mathrm{x}$-ray fluorescence experiments at the ID21 beamline at the ESRF in Grenoble, France (left). X-ray pixelsize $~ 300 \mathrm{~nm} \times 300 \mathrm{~nm}$. Comparison of the data with TEM images (right) enables an accurate identification of tissues, cells and subcellular structures. Scale Bar $=5 \mu \mathrm{m}$.

The recorded signal is clean enough to enable a discrimination between the individual tissues and subcellular structures, especially when focusing on single elements (Figure 48).
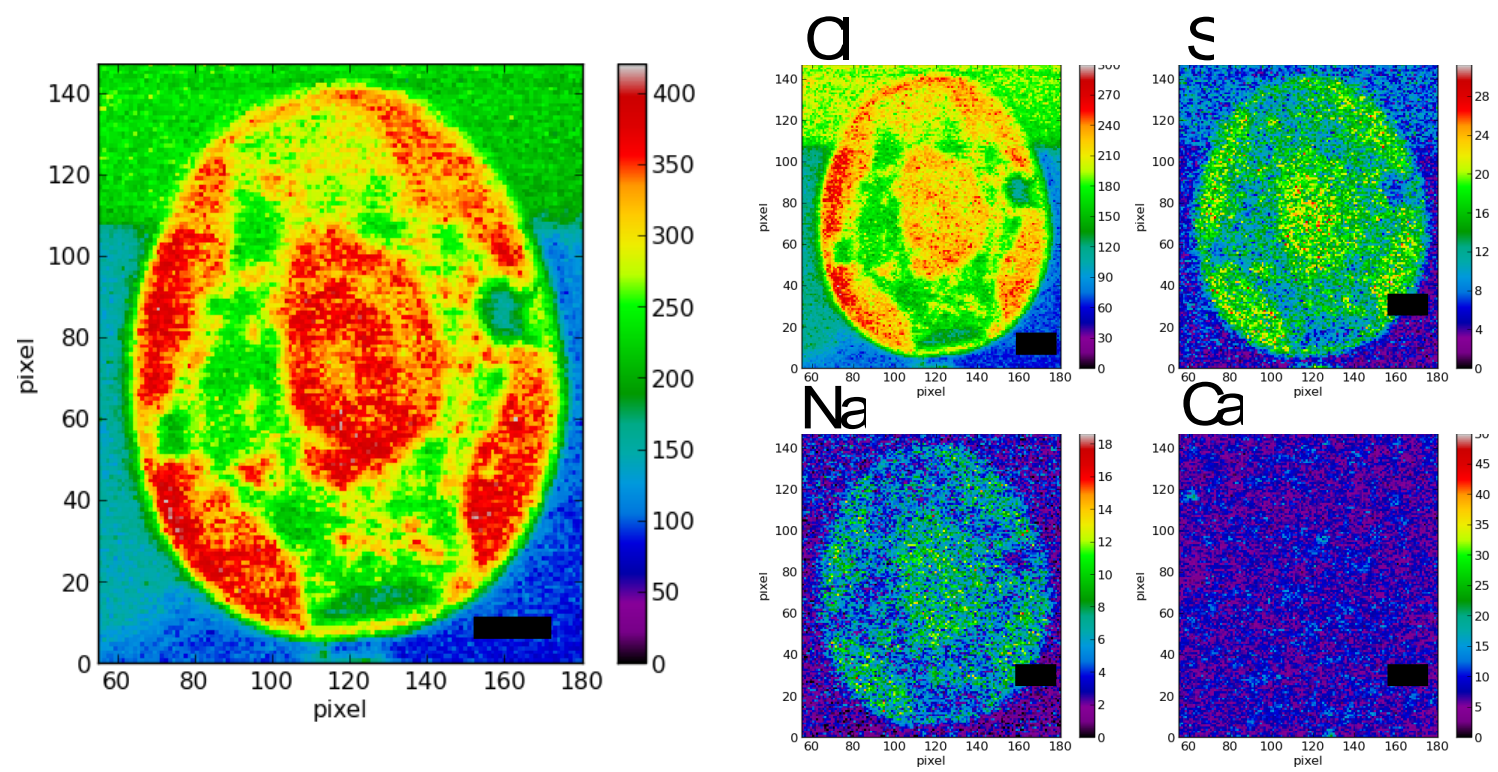

Figure 48: Elemental distribution maps of a ultrathin epon slice including a nematode cross-section

The elemental distribution maps,generated with PyMCA, display element specific fractions of recorded data according to the fitted elemental profile (compare Fig 43). Scale $=$ intensity of the recorded signal in arbitrary units (AU). Left: Sum of all relevant is displayed. Right: Elemental distribution maps of chlorine (Cl), sulphur (S), sodium $(\mathrm{Na})$ and calcium $(\mathrm{Ca})$ are depicted. Scale bar $=8 \mu \mathrm{m}$. 
The elements contributing the most structural information for the thin epon-sections was chlorine while most other distribution such as for example sulfur and sodium were largely homogenous. As the embedding procedures flush all cytosolic ions the detection of a prominent calcium signal within the fitted data curve was unexpected (compare Fig. 51). However, the contributions towards the recorded signals is low and largely arises from the embedding media and thus the distribution of calcium was largely homogenous (compare Figure 53). The possibility of imaging of calcium levels in worms has however not been eradicated completely. By turning to worms expressing photoactivatable-calcium-transporter in various mutant backgrounds and the use of calcium free embedding media in combination or vitrified cryo-sections a detection might be possible. Nevertheless the thin sections proved to be a feasible object for XRF studies and a combination of tomography and fluorescence analysis could lead to 3 dimensional elemental distribution maps in the future.

It should also be noted that the osmium, used during samples fixation, gave rise to multiple signals, which were covering large parts of the signals of less abundant elements (Figure 49). Furthermore, it may be noted that an accumulation of chloride as an effect of beam "damage" was detected when taking duplicate scans. Possible chemical reactions with the environment upon x-ray exposure might be a reason for this. Further optimization of the embedding protocols is recommended, as this method has proven its applicability and potential.
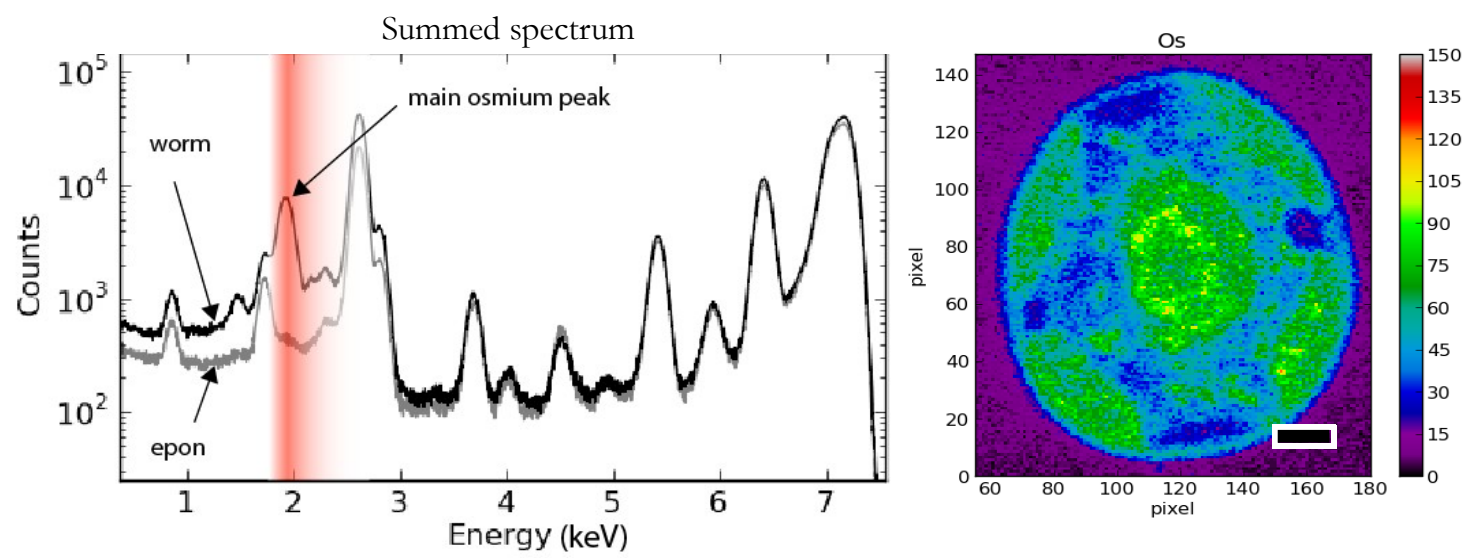

Figure 49: Osmium signal contribution

Left: A comparison of the surrounding embedding media to the nematode tissue reveals a large osmium accumulation in the tissue. Right: The elemental distribution map of osmium (Os) shows the preference of osmium to stain lipid containing structures. Membrane rich structures such as muscles and mitochondria give rise to a very strong signal. Unfortunately the signal peak is very broad (red) and thus covers lots of other elements. Scale bar $=8 \mu \mathrm{m}$. 


\section{Discussion}

\section{The system has proven its applicability}

In our preliminary studies we have demonstrated the applicability of $\mathrm{x}$-ray imaging strategies on high-pressure frozen, slow-substituted and plastic-embedded specimen, for holographic imaging, x-ray fluorescence and phase contrast tomography.

All of the used imaging strategies can clearly visualize the sample structures and may in the future be used to obtain structural information complementary to classical TEM studies. To this end, particular advances in the x-ray analysis are the recording of elemental distribution maps derived from fluorescence imaging and the possibility for the enlarged sample size which allowed to visualize complete worm in subcellular resolving tomography studies.

\section{Several adjustments can be made to improve the obtained results}

Our preliminary studies line out both the potential and the chalanges particular of novel x-ray imaging tools. In particular, they point towards possible adjustments that can be made in order to improve the results of our studies. These modifications can be categorized in three classes.

Firstly, the imaging setups and experiments can be modified to perform in a more suitable fashion for the TEM samples. To point out only one example one can highlight the presence of high resolving Fresnel-Zone-Plates (FZPs) at the ID21 beamline. The resolution of the used Zone-Plate was $330 \mathrm{~nm}$ while $100 \mathrm{~nm}$ Zone-Plates were already available at this time. Due to the limited beamtime, a rearrangement and thus recalibration of the optics, to account for the higher resolving plate, was not possible. Considering that the TEM slices themselves had a thickness of $500 \mathrm{~nm}$ we can however assume a uniform voxel size when imaging with the $330 \mathrm{~nm}$ plate $(330 \times 330 \times 500 \mathrm{~nm})$. In a future attempt one would definitely use the best FZP which in combination with an according slice-thickness would lead to the smallest but still uniform voxel size. With the preliminary results from the performed experiments an application for a dedicated beamtime seems to be very promising.

Secondly, the sample preparation needs to be discussed. All investigations have been performed using the TEM samples, processed in the - for the field - standardized fashion. However, these preparations have several shortcoming when used in the performed $\mathrm{x}$-ray experiments. As an example, on the one hand the heavy metal ions osmium and lead are used 
to fix and to produce a higher contrast for the electron microscopic studies. On the other hand these elements are largely covering the signals of other trace elements in the fluorescence studies. This complicates the proper identification of elements that are not so abundant in the sample but emit at close-by energies. Moreover, the tomographic imaging could have been improved by using a fully free-standing nematode rather than a foil embedded worm. The borders or the foil create very large phase shifts that reduce the quality of the entire data set as they create artifacts in the reconstruction. Finally, as already mentioned, the slice thickness of the imaged samples needs to be chosen in accordance with the experimental setups used.

The final issue for improvements that has to be raised are the advancements in the technical aspects of the experiments. This field has seen dramatic improvements within the past and over the last two years, from when this project was initiated, the focusing capabilities with KB mirrors, Fresnel-Zone-Plates or Compound-Refrective-Lenses have been lowered down to size of $30 \mathrm{~nm} \times 30 \mathrm{~nm}$ (Chen et al, 2008, Timmann et al., 2009) in some setups and continue to shrink even more. The introduction of photon channeling waveguides for hard $\mathrm{x}$-rays will hopefully boost this process even further (Krüger et al., 2010). To sum up, this means that our imaging attempts were performed at a resolutions below the high-end standards of available optical setups. Therefore, the improvements of the beamlines itself will help to obtain even better results in the future. As our data can already depict intracellular organelles, future imaging studies may even reveal subcellular structures like Golgis or vesicles.

In addition to the improvement of optics, the present algorithms have also undergone drastic improvements (Giewekemeyer, 2001). As an additional note the reader has to be aware that all previously mentioned reconstructions are based on a full coherent x-ray beam, but the presented holograms have been recorded under the condition of partial coherence (Williams et al., 2007). As it is very difficult to tune the experimental setup in this respect, the algorithmic side may have to take care of this phenomenon in the future. According experimental studies in this respect will be tested soon.

\section{Native states imaging is rendered possible for the future}

As these studies were initially designed to be performed with the average TEM section and samples we have neglected that a considerable beam damage might have been dealt to our samples. Hence, in a complete non-invasive approach one would have to optimize the 
experimental setups and conditions accordingly since we have seen, especially in the $\mathrm{x}$-ray fluorescence studies that we have caused damage to the thin sliced specimen.

However, the tomography samples have had negligible damage after long term imaging and a further processing, for example by trimming to an identified region of interest for electron tomographic approaches seems to be possible. In the currently developed nanoscope setups (Larabell and LeGros, 2003; Stampanoni et al., 2010) for the region-of-interest-topographies the final resolutions will soon be pushed into the nanometer range which could resolve subcellular contents. Conducting tomography on samples that lack osmium staining during the embedding and are prepared as free standing nematodes (samples that are already prepared and are now waiting for experimental synchrotron beamtimes) offers the observation of a far more native tissue in the future. This standard might even compete with TEM imaging and TEM tomography in the next decades.

In summary, the system of TEM embedding procedures has proven to be adequate when it comes to preservation of biological materials for x-ray imaging studies in a near native state. And, as the ultra-rapid fixation by high pressure freezing leaves the option of direct imaging under cryogenic condition, the frozen-native state may be visualized in the future, once beamlines will be equipped with cryogenic chambers and imaging of frozen-vitrified samples is possible. 


\section{Concluding remarks}

The presented thesis work is organized around three themes related to intracellular trafficking. At first, we conducted the morphological and behavioral characterizations of the rab mutants in C. elegans with the help of HPF-EM to understand the general role of rabs in intracellular trafficking. The second focus was directed towards the ultra-fast trafficking events at the neuromuscular synapses, where we introduced the novel electron micrograph analysis tool XtraCOunt to facilitate vesicle distribution studies. The final aim was to overcome the limitations of EM analysis by exploring the possibilities that imaging with x-rays can offer for high resolution studies.

Having established the methods for near-native preservation for EM by high-pressure freezing and slow-substitution, the fundamental morphologic analysis of the available C. elegans rab mutants was initiated. rabs are expressed tissue-specific and RABs localize compartmentspecific. For this reason, serial reconstructions of entire tissues and tomographic reconstruction of subcellular structures were generated for wild type and mutant worms along with standard $2 \mathrm{D}$ imaging. These results were thus combined in the Rab-Atlas. This atlas is, together with the findings of several behavioral and molecular biological studies, included in the Rab-Wiki. The Wiki, which upon completion, should function as an open access platform to facilitate future trafficking studies and to foster collaborations. It enables fast but encompassing integration and presentation of all available data for each member of this family of elementary trafficking regulators. The generation of this platform for the good of the trafficking community represents a major contribution of this work.

As most rabs show neuronal expression and HPF-EM instantaneously vitrifies a given sample tissue, the ultra-fast trafficking events in neuromuscular synapses were investigated. Mutants affecting synaptic trafficking were previously characterized by time-consuming and error-prone methods that characterize the vesicle distribution within the terminal. To facilitate these studies, the semi-automatic image analysis tool XtraCOunt was developed. The program is able to enhance the speed of the analysis and produce robust results. In comparison to manual methods, it allows a deeper analysis of the micrographs by not only presenting the vesicle distribution but by allowing the focus on sub-populations and special distance measurements. Here XtraCOunt was used to characterize the potentially neuronally impaired rab mutant rab- 
10, where an increase in dense-core-vesicle size was detected. In addition to pure vesicle related studies, this program can be applied in a versatile fashion to many imaging studies (as an example the characterization of $d p y-1$ mutant worms is shown in this thesis). Due to widespread applicability to other studies, XtraCOunt represents the second effort that is hopefully able to boost the efficiency of the intracellular trafficking community.

The final part of the thesis attempts to enlarge the toolbox of the trafficking community even further. It demonstrates the applicability of x-ray imaging strategies on high-pressure frozen samples for holographic imaging, $\mathrm{x}$-ray fluorescence and phase contrast tomography. The experiments give rise to novel insights that are not able to be obtained with classical TEM studies. Remarkable results are the elemental distribution maps of thin-slices epon-embedded worms and the whole-worm-tomogram with sub-cellular resolution. Imaginable for the future is a combination of both methods under cryogenic conditions which would allow a direct access to structure and composition of all cells in the worm. This possibility, to study an entire multicellular organism with a fully mapped nervous system under near-native conservation at sub-cellular resolution, definitely represents a goal in the entire trafficking community. As EM studies usually suffer from size, dosage and preparation limitations the presented x-ray imaging strategies offer a great potential in this respective as these limitations are of less concern there. In summary, this preliminary study might lead to the integration of $\mathrm{x}$-ray experiments into the standard repertoire of trafficking scientists. 


\section{References}

Albertson, D. G. and J. N. Thomson (1976). "The Pharynx of Caenorhabditis elegans." Philosophical Transactions of the Royal Society of London. B, Biological Sciences 275(938): 299-325.

Alexandrov, K. (1994). "Rab escort protein-1 is a multifunctional protein that accompanies newly prenylated rab proteins to their target membranes." EMBO Journal 13(22): 5262.

Als-Nielsen, J. and D. McMorrow (2000). "Elements of Modern X-Ray Physics." John Wiley \& Sons, Hoboken, NJ .

Anselmo, S. and L. Avery (2004). eat-17 encodes a putative Rab GTPase activating protein that acts in concert with RAB-6.2 to promote proper grinder formation in C. elegans. West Coast Worm Meeting.

Audhya, A., I. X. McLeod, et al. (2007). "MVB-12, a Fourth Subunit of Metazoan ESCRT-I, Functions in Receptor Downregulation." PLoS ONE 2(9): e956.

Avery, L. and H. R. Horvitzt (1989). "Pharyngeal pumping continues after laser killing of the pharyngeal nervous system of C. elegans." Neuron 3(4): 473-485.

Bednarek, S. Y., L. Orci, et al. (1996). "Traffic COPs and the formation of vesicle coats." Trends in Cell Biology 6(12): 468-473.

Beer, A. (1852). "Bestimmung der Absorption des rothen Lichts in farbigen Flüssigkeiten." Annalen der Physik und Chemie 86: 78-88.

Bentley, M., Y. Liang, et al. (2006). "SNARE Status Regulates Tether Recruitment and Function in Homotypic COPII Vesicle Fusion." Journal of biological chemistry 281(50): 38825-38833.

Bock, J. B., H. T. Matern, et al. (2001). "A genomic perspective on membrane compartment organization." Nature 409(6822): 839-841.

Bonifacino, J. S. and J. H. Hurley (2008). "Retromer." Current Opinion in Cell Biology 20(4): 427-436.

Bouguer, P. (1729). "Essai d'optique sur la gradation de la lumiere." C. Jombert, Paris reprinted in: Les maitres de la pensee scientique, Paris, 1921.

Branicky, R. and S. Hekimi (2006). "What keeps C. elegans regular: the genetics of defecation." Trends in Genetics 22(10): 571-579.

Brodsky, F. M. (1988). "Living with clathrin: its role in intracellular membrane traffic." Science 242(4884): 13961402.

Bronnikov, A. V. (1999). "Reconstruction formulas in phase-contrast tomography." Optics Communications 171(4-6): 239-244.

Bronnikov, A. V. (2002). "Theory of quantitative phase-contrast computed tomography." J. Opt. Soc. Am. A 19(3): 472-480.

Brunger, A. T. (2005). "Structure and function of SNARE and SNARE-interacting proteins." Quarterly Reviews of Biophysics 38(01): 1-47.

Burhop, E. H. S. (1955). J. Phys. Rad. 16: 625.

Byerly, L., R. C. Cassada, et al. (1976). "The life cycle of the nematode Caenorhabditis elegans : I. Wild-type growth and reproduction." Developmental Biology 51(1): 23-33.

C. elegans Sequencing Consortium (1998). "Genome Sequence of the Nematode C. elegans: A Platform for Investigating Biology." Science 282(5396): 2012-2018.

Cai, H., K. Reinisch, et al. (2007). "Coats, Tethers, Rabs, and SNAREs Work Together to Mediate the Intracellular Destination of a Transport Vesicle." Developmental Cell 12(5): 671-682. 
Carroll, K., J. Hanna, et al. (2001). "Role of Rab9 GTPase in facilitating receptor recruitment by TIP47." Science. : 1373-6.

Chavrier, P., R. G. Parton, et al. (1990). "Localization of low molecular weight GTP binding proteins to exocytic and endocytic compartments." 62(2): 317-329.

Chen, C. C.-H., P. J. Schweinsberg, et al. (2006). "RAB-10 Is Required for Endocytic Recycling in the Caenorhabditis elegans Intestine." Mol. Biol. Cell 17(3): 1286-1297.

Chen, Y. and et al. (2008). "Full-field hard x-ray microscopy below $30 \mathrm{~A} \mathrm{~nm}$ : a challenging nanofabrication achievement." Nanotechnology 19(39): 395302.

Cheng, J. and S. Han (1999). "On X-ray in-line Gabor holography with a partially coherent source." Optics Communications 172(1-6): 17-24.

Christoforidis, S., M. Miaczynska, et al. (1999). "Phosphatidylinositol-3-OH kinases are Rab5 effectors." Nat Cell Biol 1(4): 249-252.

Cloetens, P. and et al. (1996). "Phase objects in synchrotron radiation hard x-ray imaging." Journal of Physics D: Applied Physics 29(1): 133.

Dal Santo, P., M. A. Logan, et al. (1999). "The Inositol Trisphosphate Receptor Regulates a 50-Second Behavioral Rhythm in C. elegans." Cell 98(6): 757-767.

de Wit, H. (2010). "Molecular mechanism of secretory vesicle docking." Biochem Soc Trans. 38(Pt 1): 6.

Deinhardt, K., S. Salinas, et al. (2006). "Rab5 and Rab7 Control Endocytic Sorting along the Axonal Retrograde Transport Pathway." 52(2): 293-305.

Delprato, A., E. Merithew, et al. (2004). "Structure, Exchange Determinants, and Family-Wide Rab Specificity of the Tandem Helical Bundle and Vps9 Domains of Rabex-5." 118(5): 607-617.

Dent, J. A., M. W. Davis, et al. (1997). "avr-15 encodes a chloride channel subunit that mediates inhibitory glutamatergic neurotransmission and ivermectin sensitivity in Caenorhabditis elegans." EMBO J 16(19): 58675879.

Di Giovanni, S., C. D. Knights, et al. (2006). "The tumor suppressor protein p53 is required for neurite outgrowth and axon regeneration." EMBO J 25(17): 4084-4096.

Ducic, T., S. Quintes, et al. "Structure and composition of myelinated axons: A multimodal synchrotron spectromicroscopy study." Journal of Structural Biology 173(2): 202-212.

Duden, R., G. Griffiths, et al. (1991). "[beta]-COP, a $110 \mathrm{kd}$ protein associated with non-clathrin-coated vesicles and the golgi complex, shows homology to [beta]-adaptin." Cell 64(3): 649-665.

Echard, A., F. Jollivet, et al. (1998). "Interaction of a Golgi-Associated Kinesin-Like Protein with Rab6." Science 279(5350): 580-585.

Edgar, R. (2004). "MUSCLE: a multiple sequence alignment method with reduced time and space complexity." BMC Bioinformatics 5(1): 113 .

Espelt, M. V., A. Y. Estevez, et al. (2005). "Oscillatory Ca2+ Signaling in the Isolated Caenorhabditis elegans Intestine." The Journal of General Physiology 126(4): 379-392.

Falcone, S., E. Cocucci, et al. (2006). "Macropinocytosis: regulated coordination of endocytic and exocytic membrane traffic events." J Cell Sci: jcs.03238.

Fasshauer, D., B. R. Sutton, et al. (1998). "Conserved structural features of the synaptic fusion complex: SNARE proteins reclassified as Q- and R-SNAREs." Proc Natl Acad Sci U S A 95(26): 6.

Fath, S., J. D. Mancias, et al. (2007). "Structure and Organization of Coat Proteins in the COPII Cage." 129(7): $1325-1336$.

Fukuda, M. (2008). "Membrane traffic in the secretory pathway." Cellular and Molecular Life Sciences 65(18): 2801-2813.

Gabor, D. (1948). " A new microscopic principle." Nature 161: 777-778.

Gamulin, V., I. M. Muller, et al. (2000). "Sponge proteins are more similar to those of Homo sapiens than to 
Caenorhabditis elegans." Biological Journal of the Linnean Society 71(4): 821-828.

Gamulin, V., I. M. Muller, et al. (2000). "Sponge proteins are more similar to those of Homo sapiens than to Caenorhabditis elegans." Biological Journal of the Linnean Society 71(4): 821-828.

Gerchberg, R. W. and W. O. Saxton (1972). "A practical algorithm for the determination of the phase from image and diffraction plane pictures." Optik 35: 237.

Ghazi-tabatabai, S., T. Obita, et al. (2009). "Evolution and assembly of ESCRTs." Biochemical Society Transactions 037(1): 151-155.

Ghazi-Tabatabai, S., S. Saksena, et al. (2008). "Structure and Disassembly of Filaments Formed by the ESCRT-III Subunit Vps24." Structure 16(9): 1345-1356.

Giewekemeyer, K. (2011). "A study on new approaches in coherent x-ray microscopy of biological specimens." Doctoral thesis.

Giewekemeyer, K., S. P. Krüger, et al. (2011). "X-ray propagation microscopy of biological cells using waveguides as a quasipoint source." Physical Review A 83(2): 023804.

Giewekemeyer, K., P. Thibault, et al. (2010). "Quantitative biological imaging by ptychographic x-ray diffraction microscopy." Proceedings of the National Academy of Sciences 107(2): 529-534.

Goody, R. S. (2005). "The structural and mechanistic basis for recycling of Rab proteins between membrane compartments." Cellular and molecular life sciences 62(15): 1657.

Groso, A., M. Stampanoni, et al. (2006). "Phase contrast tomography: An alternative approach." Applied Physics Letters 88(21): 214104-3.

Grosshans, B. L., D. Ortiz, et al. (2006). "Rabs and their effectors: Achieving specificity in membrane traffic." Proceedings of the National Academy of Sciences 103(32): 11821-11827.

Gurkan, C., S. M. Stagg, et al. (2006). "The COPII cage: unifying principles of vesicle coat assembly." Nat Rev Mol Cell Biol 7(10): 727-738.

Hall, T. (1961). "X-ray Fluorescence Analysis in Biology." Science 134(3477): 449-455.

Hanson, P. I., J. E. Heuser, et al. (1997). "Neurotransmitter release -- four years of SNARE complexes." Current Opinion in Neurobiology 7(3): 310-315.

Hanson, P. I., R. Roth, et al. (2008). "Plasma membrane deformation by circular arrays of ESCRT-III protein filaments." The Journal of Cell Biology 180(2): 389-402.

Henke, B. L., E. M. Gullikson, et al. (1993). "X-Ray Interactions: Photoabsorption, Scattering, Transmission, and Reflection at E = 50-30,000 eV, Z = 1-92." Atomic Data and Nuclear Data Tables 54(2): 181-342.

Hierro, A., A. L. Rojas, et al. (2007). "Functional architecture of the retromer cargo-recognition complex." Nature 449(7165): 1063-1067.

Hillier, L. W., A. Coulson, et al. (2005). "Genomics in C. elegans: So many genes, such a little worm." Genome Research 15(12): 1651-1660.

Hirst, J., N. A. Bright, et al. (1999). "Characterization of a Fourth Adaptor-related Protein Complex." Mol. Biol. Cell 10(8): 2787-2802.

Hoepfner, S., F. Severin, et al. (2005). "Modulation of Receptor Recycling and Degradation by the Endosomal Kinesin KIF16B." Cell 121(3): 437-450.

Hoppert, M. (2003). "Microscopic techniques in biotechnology." Wiley.

Hosono, R., K. Hirahara, et al. (1982). "Mutants of Caenorhabditis elegans with dumpy and rounded head phenotype." Journal of Experimental Zoology 224(2): 135-144.

Javier, P.-C., B. Matteo, et al. "Clathrin-mediated endocytosis: What works for small, also works for big." BioEssays 32(6): 496-504.

Jena, B. P. and P. J. Bhanu (2008). Chapter 8 Assembly and Disassembly of SNAREs in Membrane Fusion. Methods in Cell Biology, Academic Press. Volume 90: 157-182. 
Jordens, I., M. Fernandez-Borja, et al. (2001). "The Rab7 effector protein RILP controls lysosomal transport by inducing the recruitment of dynein-dynactin motors." Current Biology 11(21): 1680-1685.

Kak, A. C. and M. Slaney (1988). Principles of computerized tomographic imaging.

Kalbfleisch, S., M. Osterhoff, et al. (2010). The holography endstation of beamline P10 at PETRA III. SRI 2009, 10TH INTERNATIONAL CONFERENCE ON RADIATION INSTRUMENTATION, Melbourne (Australia), AIP.

Kamin, D., M. A. Lauterbach, et al. "High- and Low-Mobility Stages in the Synaptic Vesicle Cycle." Biophysical Journal 99(2): 675-684.

Katzmann, D. J., M. Babst, et al. (2001). "Ubiquitin-Dependent Sorting into the Multivesicular Body Pathway Requires the Function of a Conserved Endosomal Protein Sorting Complex, ESCRT-I." 106(2): 145-155.

Klein, A., C. Olendrowitz, et al. (2009). "Identification of brain- and bone-specific breast cancer metastasis genes." Cancer letters 276(2): 212-220.

Kloepper, T. H., C. N. Kienle, et al. (2007). "An Elaborate Classification of SNARE Proteins Sheds Light on the Conservation of the Eukaryotic Endomembrane System." Mol. Biol. Cell 18(9): 3463-3471.

König, S., M. Hoffmann, et al. (2008). "Determination of content and fatty acid composition of unlabeled phosphoinositide species by thin-layer chromatography and gas chromatography." Analytical Biochemistry 378(2): 197-201.

König, S., M. Hoffmann, et al. (2008). "Determination of content and fatty acid composition of unlabeled phosphoinositide species by thin-layer chromatography and gas chromatography." Analytical Biochemistry 378(2): 197-201.

Krüger, S. P. (2010). "Optimization of waveguide optics for lensless x-ray imaging." Doctoral thesis.

Krüger, S. P., K. Giewekemeyer, et al. (2010). "Sub-15 nm beam confinement by twocrossed x-ray waveguides." Opt. Express 18(13): 13492-13501.

Kummel, D. and U. Heinemann (2008). "Diversity in Structure and Function of Tethering Complexes: Evidence for Different Mechanisms in Vesicular Transport Regulation." Current Protein and Peptide Science 9: 197-209.

Kwan, C., R. Vazquez-Manrique, et al. (2008). "TRPM channels are required for rhythmicity in the ultradian defecation rhythm of C. elegans." BMC Physiology 8(1): 11.

Lambert, J. H. (1760). "Photometria, sive de mensura et gradibus luminis, colorum et umbrae." Sumptibus Vidae Eberhardi Klett.

Larabell, C. A. and M. A. Le Gros (2004). "X-ray Tomography Generates 3-D Reconstructions of the Yeast, Saccharomyces cerevisiae, at 60-nm Resolution." Mol. Biol. Cell 15(3): 957-962.

Lindner, R. and E. Ungewickell (1992). "Clathrin-associated proteins of bovine coated vesicles." Journal of biological chemistry 267(23): 7.

Lipowsky, R. and E. Sackmann (1995). "Structure and dynamics of membranes." 1.

Liu, D. and J. Thomas (1994). "Regulation of a periodic motor program in C. elegans." The Journal of Neuroscience 14(4): 1953-1962.

Lu, J., M. Machius, et al. (2006). "Structural Basis for a Munc13-1 Homodimer to Munc13-1/RIM Heterodimer Switch." PLoS Biol 4(7): e192.

Mahoney, T. R., Q. Liu, et al. (2006). "Regulation of Synaptic Transmission by RAB-3 and RAB-27 in Caenorhabditis elegans." Mol. Biol. Cell 17(6): 2617-2625.

Marza, E., T. Long, et al. (2008). "Polyunsaturated Fatty Acids Influence Synaptojanin Localization to Regulate Synaptic Vesicle Recycling." Mol. Biol. Cell 19(3): 833-842.

Matanis, T., A. Akhmanova, et al. (2002). "Bicaudal-D regulates COPI-independent Golgi-ER transport by recruiting the dynein-dynactin motor complex." Nat Cell Biol 4(12): 986-992.

Matsui, Y., A. Kikuchi, et al. (1990). "Molecular cloning and characterization of a novel type of regulatory protein (GDI) for smg p25A, a ras p21-like GTP-binding protein." Mol. Cell. Biol. 10(8): 4116-4122. 
McDonald, B. and J. Martin-Serrano (2009). "No strings attached: the ESCRT machinery in viral budding and cytokinesis." J Cell Sci 122(13): 2167-2177.

McLauchlan, H., J. Newell, et al. (1998). "A novel role for Rab5 GDI in ligand sequestration into clathrin-coated pits." 8(1): 34-45.

McMahon, H. T. and I. G. Mills (2004). "COP and clathrin-coated vesicle budding: different pathways, common approaches." Current Opinion in Cell Biology 16(4): 379-391.

McMahon, L., J. M. Muriel, et al. (2003). "Two Sets of Interacting Collagens Form Functionally Distinct Substructures within a Caenorhabditis elegans Extracellular Matrix." Mol. Biol. Cell 14(4): 1366-1378.

McMahon, L., J. M. Muriel, et al. (2003). "Two Sets of Interacting Collagens Form Functionally Distinct Substructures within a Caenorhabditis elegans Extracellular Matrix." Mol. Biol. Cell 14(4): 1366-1378.

Menasche, G., E. Pastural, et al. (2000). "Mutations in RAB27A cause Griscelli syndrome associated with haemophagocytic syndrome." Nat Genet 25(2): 173-176.

Mettlen, M., T. Pucadyil, et al. (2009). "Dissecting dynamin's role in clathrin-mediated endocytosis." Biochemical Society Transactions 037(5): 1022-1026.

Miguel, C. S. and C. Evelyne (2004). "Rab GTPases and Myosin Motors in Organelle Motility." Traffic 5(6): 393399.

Miller, D. M., D. C. Shakes, et al. (1995). Chapter 16 Immunofluorescence Microscopy. Methods in Cell Biology, Academic Press. Volume 48: 365-394.

Morita, E., V. Sandrin, et al. (2007). "Human ESCRT and ALIX proteins interact with proteins of the midbody and function in cytokinesis." EMBO J 26(19): 4215-4227.

Moseley, H. G. J. (1913). "The frequency spectra of the elements." Phil. Mag.: 1024.

Nakatsu, F. and H. Ohno (2003). "Adaptor Protein Complexes as the Key Regulators of Protein Sorting in the Post-Golgi Network." Cell Structure and Function 28(5): 419-429.

Nonet, M. L., J. E. Staunton, et al. (1997). "Caenorhabditis elegans rab-3 Mutant Synapses Exhibit Impaired Function and Are Partially Depleted of Vesicles." J. Neurosci. 17(21): 8061-8073.

Nugent, K. A. (1990). "Twin-image elimination in Gabor holography." Optics Communications 78(3-4): 293-299.

Nugent, K. A. (2009). "Coherent methods in x-ray science." Advances in Physics.

Nugent, K. A., T. E. Gureyev, et al. (1996). "Quantitative Phase Imaging Using Hard X Rays." Physical Review Letters 77(14): 2961.

Ohno, H., J. Stewart, et al. (1995). "Interaction of tyrosine-based sorting signals with clathrin-associated proteins." Science 269(5232): 1872-1875.

Paganin, D. M. (2006). "Coherent X-ray Optics." Oxford University Press.

Palade, G. (1975). "Intracellular aspects of the process of protein synthesis." Science 189(4200): 347-358.

Pan, X., S. Eathiraj, et al. (2006). "TBC-domain GAPs for Rab GTPases accelerate GTP hydrolysis by a dualfinger mechanism." Nature 442(7100): 303-306.

Pearse, B. (1980). "Coated vesicles." Trends in Biochemical Sciences 5(5): 131-134.

Pearse, B. M. F. and M. S. Bretscher (1981). "Membrane Recycling by Coated Vesicles." Annual Review of Biochemistry 50(1): 85-101.

Pearse, B. M. F. and M. S. Robinson (1990). "Clathrin, Adaptors, and Sorting." Annual Review of Cell Biology 6(1): 151-171.

Pereira-Leal, J. B. and M. C. Seabra (2001). "Evolution of the rab family of small GTP-binding proteins." Journal of Molecular Biology 313(4): 889-901.

Peters, M. A., T. Teramoto, et al. (2007). "A Calcium Wave Mediated by Gap Junctions Coordinates a Rhythmic Behavior in C. elegans." Current Biology 17(18): 1601-1608.

Pfeffer, S. (2005). "A model for Rab GTPase localization." Biochem. Soc. Trans. 33(Pt 4): 627-630. 
Pfeffer, S. R. (2001). "Rab GTPases: specifying and deciphering organelle identity and function." Trends in Cell Biology 11(12): 487-491.

Pfeffer, S. R. (2007). "Unsolved Mysteries in Membrane Traffic." Annual Review of Biochemistry 76(1): 629-645.

Pogany, A., D. Gao, et al. (1997). "Contrast and resolution in imaging with a microfocus x-ray source." Review of Scientific Instruments 68(7): 2774-2782.

Pucadyil, T. J. and S. L. Schmid (2009). "Conserved Functions of Membrane Active GTPases in Coated Vesicle Formation." Science 325(5945): 1217-1220.

Radon, J. (1917). "Bestimmung von Funktionen durch ihre Integralwerte längs gewisser Mannigfaltigkeiten." Berichte Sächsische Akademie der Wissenschaften, Leipzig.

Raizen, D. M., R. Lee, et al. (1995). "Interacting Genes Required for Pharyngeal Excitation by Motor Neuron MC in Caenorhabditis elegans." Genetics 141(4): 1365-1382.

Reynolds, E. S. (1963). "The use of lead citrate at high pH as an electron-opaque stain in electron microscopy." The Journal of Cell Biology 17(1): 208-212.

Riddle, Blumenthal, et al. (1997). " C. elegans II." Cold Spring Harbor Laboratory Press, New York.

Rizo, J. and C. Rosenmund (2008). "Synaptic vesicle fusion." Nat Struct Mol Biol.

Rosenmund, C., J. Rettig, et al. (2003). "Molecular mechanisms of active zone function." Current Opinion in Neurobiology 13(5): 509-519.

Rostaing, P., E. Real, et al. (2006). "Analysis of synaptic ultrastructure without fixative using high-pressure freezing and tomography." European Journal of Neuroscience 24(12): 3463-3474.

Rostaing, P., R. M. Weimer, et al. (2004). "Preservation of Immunoreactivity and Fine Structure of Adult C. elegans Tissues Using High-pressure Freezing." J. Histochem. Cytochem. 52(1): 1-12.

Roux, A. 1., K. Uyhazi, et al. (2006). "GTP-dependent twisting of dynamin implicates constriction and tension in membrane fission." Nature 441(7092): 528-531.

Sasidharan, N. (2011). "Analysis of the RAB family of GTPases in C. elegans and their role in regulating neuronal membrane trafficking." Doctoral thesis.

Saxton, W. O., W. Baumeister, et al. (1984). "Three-dimensional reconstruction of imperfect two-dimensional crystals." Ultramicroscopy 13(1-2): 57-70.

Schlichting, I., S. C. Almo, et al. (1990). "Time-resolved X-ray crystallographic study of the conformational change in Ha-Ras p21 protein on GTP hydrolysis." Nature 345(6273): 309-315.

Schlichting, I., S. C. Almo, et al. (1990). "Time-resolved X-ray crystallographic study of the conformational change in Ha-Ras p21 protein on GTP hydrolysis." Nature 345(6273): 309-315.

Schuske, K., A. A. Beg, et al. (2004). "The GABA nervous system in C. elegans." Trends in Neurosciences 27(7): 407-414.

Seabra, M. C. (1996). "Nucleotide Dependence of Rab Geranylgeranylation." Journal of Biological Chemistry 271(24): 14398-14404.

Seabra, M. S. and E. Coudrier (2004). "Rab GTPases and Myosin Motors in Organelle Motility." Traffic 5(6): 393399.

Seaman, M. N. J., J. Michael McCaffery, et al. (1998). "A Membrane Coat Complex Essential for Endosome-toGolgi Retrograde Transport in Yeast." The Journal of Cell Biology 142(3): 665-681.

Semerdjieva, S., B. Shortt, et al. (2008). "Coordinated regulation of AP2 uncoating from clathrin-coated vesicles by rab5 and hRME-6." The Journal of Cell Biology 183(3): 499-511.

Shen, J., D. C. Tareste, et al. (2007). "Selective Activation of Cognate SNAREpins by Sec1/Munc18 Proteins." Cell 128(1): 183-195.

Shin, H.-W., M. Hayashi, et al. (2005). "An enzymatic cascade of Rab5 effectors regulates phosphoinositide turnover in the endocytic pathway." The Journal of Cell Biology 170(4): 607-618.

Sivars, U., D. Aivazian, et al. (2003). "Yip3 catalyses the dissociation of endosomal Rab-GDI complexes." Nature 
425(6960): 856-859.

Snigirev, A., I. Snigireva, et al. (1995). "On the possibilities of x-ray phase contrast microimaging by coherent high-energy synchrotron radiation." Rev. Sci. Instrum. 66: 5486-5492.

Soldati, T., A. D. Shapiro, et al. (1994). "Membrane targeting of the small GTPase Rab9 is accompanied by nucleotide exchange." Nature 369(6475): 76-78.

Solé, V. A., E. Papillon, et al. (2007). "A multiplatform code for the analysis of energy-dispersive X-ray fluorescence spectra." Spectrochimica Acta Part B: Atomic Spectroscopy 62(1): 63-68.

Söllner, T., M. K. Bennett, et al. (1993). "A protein assembly-disassembly pathway in vitro that may correspond to sequential steps of synaptic vesicle docking, activation, and fusion." Cell 75(3): 409-418.

Sørensen, J. B. (2005). "SNARE complexes prepare for membrane fusion." Trends in Neurosciences 28(9): 453455.

Sørensen, J. B., K. Wiederhold, et al. (2006). "Sequential N- to C-terminal SNARE complex assembly drives priming and fusion of secretory vesicles." EMBO J 25(5): 955-966.

Stagg, S. M., C. Gurkan, et al. (2006). "Structure of the Sec13/31 COPII coat cage." Nature 439(7073): $234-238$.

Stampanoni, M., R. Mokso, et al. (2010). "Phase-contrast tomography at the nanoscale using hard x rays." Physical Review B 81(14): 140105.

Stenbeck, G., C. Harter, et al. (1993). "beta'-COP, a novel subunit of coatomer." EMBO J 12(7): 2841-2845

Stenmark, H. (2009). "Rab GTPases as coordinators of vesicle traffic." Nat Rev Mol Cell Biol 10(8): 513-525.

Sudhof, T. C. (1995). "The synaptic vesicle cycle: a cascade of proteinÂ-protein interactions." Nature 375(6533): 645-653.

Südhof, T. C. (2000). "The Synaptic VesicleCycle Revisited." Neuron 28(2): 317-320.

Südhof, T. C. (2004). "The synaptic vesicle cycle." Annual Review of Neuroscience 27(1): 509-547.

Sulston and Hodgkin (1988.). "The Nematode Caenorhabditis elegans " Cold Spring Harbor Laboratory Press, Cold Spring Harbor.

Sulston, J. E., E. Schierenberg, et al. (1983). "The embryonic cell lineage of the nematode Caenorhabditis elegans." Developmental Biology 100(1): 64-119.

Sumakovic, M., J. Hegermann, et al. (2009). "UNC-108/RAB-2 and its effector RIC-19 are involved in dense core vesicle maturation in Caenorhabditis elegans." The Journal of Cell Biology 186(6): 897-914.

Sutton, R. B., D. Fasshauer, et al. (1998). "Crystal structure of a SNARE complex involved in synaptic exocytosis at 2.4A resolution." Nature 395(6700): 347-353.

Sweitzer, S. M. and J. E. Hinshaw (1998). "Dynamin Undergoes a GTP-Dependent Conformational Change Causing Vesiculation." 93(6): 1021-1029.

Takamori, S., M. Holt, et al. (2006). "Molecular Anatomy of a Trafficking Organelle." 127(4): 831-846.

Teramoto, T. and K. Iwasaki (2006). "Intestinal calcium waves coordinate a behavioral motor program in C. elegans." Cell Calcium 40(3): 319-327.

Thein, M. C., G. McCormack, et al. (2003). "Caenorhabditis elegans exoskeleton collagen COL-19: An adultspecific marker for collagen modification and assembly, and the analysis of organismal morphology." Developmental Dynamics 226(3): 523-539.

Thibault, P., V. Elser, et al. (2006). "Reconstruction of a yeast cell from X-ray diffraction data." Acta Crystallographica Section A 62(4): 248-261.

Thomas, J. H. (1990). "Genetic Analysis of Defecation in Caenorhabditis elegans." Genetics 124(4): 855-872.

Ullrich, O., H. Stenmark, et al. (1993). "Rab GDP dissociation inhibitor as a general regulator for the membrane association of rab proteins." Journal of Biological Chemistry 268(24): 18143-18150.

Valencia, A., M. Kjeldgaard, et al. (1991). "GTPase domains of ras p21 oncogene protein and elongation factor Tu: analysis of three-dimensional structures, sequence families, and functional sites." Proceedings of the National 
Academy of Sciences of the United States of America 88(12): 5443-5447.

White, J. G., E. Southgate, et al. (1986). "The Structure of the Nervous System of the Nematode Caenorhabditis elegans." Philosophical Transactions of the Royal Society of London. B, Biological Sciences 314(1165): 1-340.

Williams, G. J., H. M. Quiney, et al. (2007). "Coherent diffractive imaging and partial coherence." Physical Review B 75(10): 104102.

Wollert, T., D. Yang, et al. (2009). "The ESCRT machinery at a glance." J Cell Sci 122(13): 2163-2166.

Zabler, S., P. Cloetens, et al. (2005). "Optimization of phase contrast imaging using hard x rays." Review of Scientific Instruments 76(7): 073705-7.

Zerial, M. and H. McBride (2001). "Rab proteins as membrane organizers." Nat Rev Mol Cell Biol 2(2): 107-117. 


\section{Curriculum vitae}

Name: Christian Olendrowitz

Born: 24.03.1983 in Königs Wusterhausen, Germany

\section{previous education:}

07/2001 High-School Montevideo High School Diploma, Montevideo, MN, USA

06/2003 Abitur Special Math Class of the Humboldt University Andreas Oberschule Friedrichshain-Kreuzberg, Berlin, Germany

undergraduate studies:

- 06/2006 BSc. Bioinformatics, Free-University of Berlin, Germany

Thesis : "Gene expression profiling focused on metastatic behavior of buman breast cancer. Development of the cluster analysis tool XtraCAT"

\section{graduate studies:}

- 03/2008 MSc.

Molecular Biology, International Max Planck Research School of the Georg August University Göttingen, Germany

Thesis "High-pressure freezing based electron microscopic analysis of rab-protein mutants in C. elegans"

\section{doctoral studies (PhD):}

Graduate Program of the DFG Research Center: Molecular Physiology of the Brain (CMPB) in the Göttingen Graduate School for Neurosciences and Molecular Biosciences (GGNB) at the Georg August University Göttingen, Germany.

04/2008-03/2010 Molecular Neurogenetics, supervised by Dr. Stefan Eimer European Neuroscience Institute, Göttingen, Germany

04/2010- present X-ray Physics, supervised by Prof. Dr. Tim Salditt Institute of X-ray Physics, Georg-August-University Göttingen, Germany

stipends:

09/2006-08/2007 Olympus fellow 06/07 of the International Max Planck Research School 
IX. Curriculum vitae 


\section{Publications}

Parts of the work presented in this thesis are based on the following publications. I want to thank all co-authors and all people acknowledged therein for the successful collaboration.

Publication in preparation:

Christian Olendrowitz, Jan Hegermann, Carolin Wichmann, Stephan Sigrist, Stefan Eimer. Fascilitation of vesicle distribution studies by the novel seminautomated image analysis tool XtraCOunt.

Sandrine Jacob*, Christian Olendrowitz*, Delphine Bernard, Joachim Muriel, Charlotte Kenning, Olivier Poupel, Danny Tuckwell, Ralf Sommer, Stefan Eimer and Bernard Lakowski. DPY-1 plays a conserved role in nematode cuticle formation.

* denotes equal contribution

Jonathan R. Mayers, Christian Olendrowitz, Amber L. Schuh, Edwin R. Chapman, Stefan Eimer, and Anjon Audhya. Multiple Roles for Hrs and STAM in Multivesicular Endosome Dynamics.

Published publications:

\# Marija Sumakovic, Jan Hegermann, Steven J. Husson, Ling Luo, Katrin Schwarze, Christian Olendrowitz, Liliane Schoofs, Janet Richmond and Stefan Eimer. UNC$108 / \mathrm{RAB}-2$ and its effector RIC-19 are involved in dense core vesicle biogenesis in C. elegans. J. Cell Biol. (2009); 186:897-914.

\# Andreas Klein, Christian Olendrowitz, Rita Schmutzler, Juergen Hampl, Peter M. Schlag, Nicolai Maass, Norbert Arnold, Ralf Wessel, Juliane Ramser, Alfons Meindl, Siegfried Scherneck, Susanne Seitz. Identification of brain- and bone-specific breast cancer metastasis genes, Cancer Lett. (2008), doi:10.1016/j.canlet.2008.11.017 